\title{
THE EFFECT OF CLOSE COLLISSIONS ON THE TWO-BODY DISTRIBUTION FUNCTION IN A PLASMA
}

DAVID ELLIS BALDWIN

\section{LOAN}
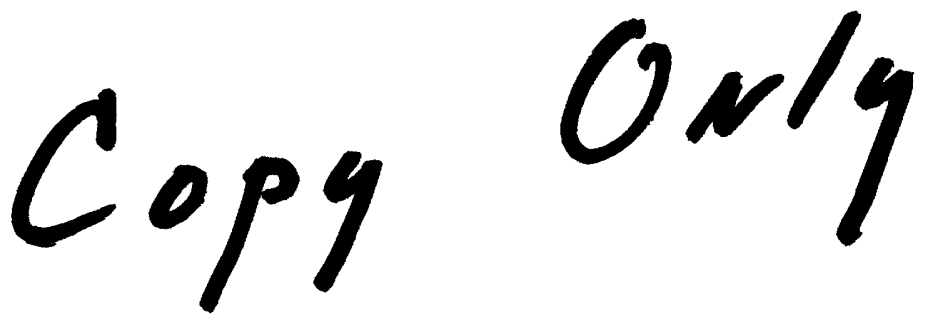

TECHNICAL REPORT 402

DECEMBER 31, 1962 
The Research Laboratory of Electronics is an interdepartmental laboratory in which faculty members and graduate students from numerous academic departments conduct research.

The research reported in this document was made possible in part by support extended the Massachusetts Institute of Technology, Research Laboratory of Electronics, jointly by the U.S. Army, the U.S. Navy (Office of Naval Research), and the U.S. Air Force (Office of Scientific Research) under Contract DA36-039-sc-78108, Department of the Army Task 3-99-25-001-08; and in part by Contract DA-SIG-36-039-61-G14; additional support was received from the National Science Foundation (Grant G-9330).

Reproduction in whole or in part is permitted for any purpose of the United States Government. 


\title{
MASSACHUSETTS INSTITUTE OF TECHNOLOGY
}

\section{RESEARCH LABORATORY OF ELECTRONICS}

\section{THE EFFECT OF CLOSE COLLISIONS ON THE TWO-BODY DISTRIBUTION FUNCTION IN A PLASMA}

\author{
David Ellis Baldwin
}

Submitted to the Department of Physics, M. I. T., May 11, 1962, in partial fulfillment of the requirements for the degree of Doctor of Philosophy.

\author{
(Manuscript received May 15, 1962)
}

\begin{abstract}
This investigation is concerned with the development of a two-body distribution function in a plasma for use in a kinetic equation for the one-body distribution function. The kinetic equation is obtained for a uniform plasma for those circumstances in which the time dependence of higher-order distribution functions can be assumed to occur within a functional dependence on the one-particle distribution function. The conditions of validity for this functional-dependence assumption are discussed. The resulting interaction term is new in the sense that it contains no divergent integrals requiring cutoffs, and it may be considered accurate to first order in $\left(e^{2} / k T \lambda_{D}\right)$. The interaction term is composed of two parts. The first is a Boltzmann collision integral with a Debye-shielded interaction. The second term is due to the deviation of the shielding cloud from a Debye shield and is the Fokker-Planck form, the coefficients of which are finite and wellbehaved. Because of its form, with a convergent collision integral and convergent Fokker-Planck coefficients, the solution may be considered as a joining of the previous solutions to this problem.
\end{abstract}


TABLE OF CONTENTS

I. The Nature of the Problem 1

1.1 Introduction 1

1.2 Statement of the Problem 4

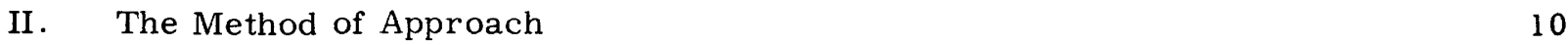

2.1 The Adiabatic Hypothesis and Its Implications 10

2.2 The Large-Separation Solution $\quad 14$

2.3 Collision Problem Assumptions 17

III. Collision Problem Solution 19

3.1 The Equation for $\mathrm{F}_{3}^{1} \quad 19$

3.2 Solution of the Equation for $\mathrm{F}_{3}^{1}$

IV. Discussion of Results 39

4.1 The Equilibrium Case 39

$\begin{array}{lll}4.2 & \text { The Nonequilibrium Case } & 43\end{array}$

V. Summary

$\begin{array}{ll}\text { Appendix } & 53\end{array}$

$\begin{array}{ll}\text { Acknowledgment } & 56\end{array}$

$\begin{array}{ll}\text { References } & 57\end{array}$ 


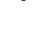

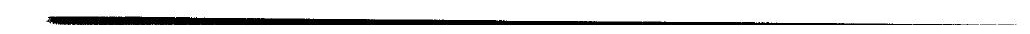




\section{THE NATURE OF THE PROBLEM}

\subsection{INTRODUCTION}

In the description of the evolution of a fluid, the interplay of the properties of the interaction and the density determine whether the fluid is a liquid, gas or plasma. If at the interparticle distance the motions of the particles are strongly affected by the forces that are due to other particles, we consider the fluid a liquid. Forces that are weak at the interparticle distance will give rise to fluids described as gases or plasmas. If we consider forces that fall off at large distances to be $r^{-a}$, we may distinguish gases and plasmas as $a>2$ and $a \leqslant 2$, respectively. For gases the effect of the volume element in a summation over particles is not enough to counteract the $r^{-a}$-decay of the force. The summation will receive its greatest contribution from near particles. In a gas of this type, for a density that is sufficiently low that particles interact only infrequently, we are led to the concept of a two-body collision and a Boltzmann gas. For $a \leqslant 2$, the $r^{2}$-increase of the volume element is sufficient to increase the effect of the more distant particles. In particular, for Coulomb interactions in a plasma the volume element just offsets the $\mathrm{r}^{-2}$-decay, and the concept of a collision becomes vague, since many particles interact at once.

Kirkwood and Poirier ${ }^{l}$ show that for a plasma in equilibrium the effect of the Coulomb potential is modified by the screening of other particles and an effective potential is established. The effective potential can be well approximated by the Debye potential, $\frac{\mathrm{e}}{\mathrm{r}} \mathrm{e}^{-\mathrm{r} / \lambda_{\mathrm{D}}}$. The Debye length, $\lambda_{D}=\sqrt{\frac{\mathrm{kT}}{4 \pi n \mathrm{e}^{2}}}$, for $\mathrm{n}$ particles per unit volume, becomes a new range of interaction. As we shall see (sec. 1.2), plasmas of interest will be of such a density that they will have many particles within a radius $\lambda_{D} ; n \lambda_{D}^{3}$ is a large number. We are still faced with many particles interacting at once.

Because the information that is eventually desired about a gas will not depend on the detailed motion of particular particles, but rather on an average over many particles, it is convenient to introduce distribution functions. Thus we introduce the distribution functions

$$
F_{1}\left(x_{1} ; t\right), F_{2}\left(x_{1}, x_{2} ; t\right), \ldots, F_{s}\left(x_{1}, \ldots x_{s} ; t\right),
$$

where the subscript denotes the number of particles in the argument; $t$, the time; and $x_{i}$, the 6-dimensional vector $\left\{\vec{p}_{i}, \vec{q}_{i}\right\}$. We shall assume that the $F_{s}$ are invariant under an interchange of particles, so that the particular $x_{i}$ appearing in the arguments are simply labels. The $F_{s}$ may be defined as follows: The function $\frac{1}{V^{s}} F_{s}\left(x_{1}, \ldots x_{s} ; t\right) d x_{1}$ $\ldots \mathrm{dx}_{\mathrm{s}}$ is the probability at time $\mathrm{t}$ that the particles $1 \ldots s$ will be found, respectively, at $\mathrm{x}_{1}, \ldots \mathrm{x}_{\mathrm{s}}$ within $\mathrm{dx_{1 }} \ldots \mathrm{dx_{s }}$. We shall take the relation between the distribution functions to be

$$
F_{s}\left(x_{1}, \ldots x_{s} ; t\right)=\frac{1}{v^{k-s}} \int F_{k}\left(x_{1} \ldots x_{k}, t\right) d x_{k} \ldots d x_{s+1} k \geqslant s
$$


where the volume $\mathrm{V}$ that is available to the gas is inserted in both cases to allow a smooth passage to infinite volume. For each $\mathrm{dx}_{i}$, the integration is over all momentum space and the volume $\mathrm{V}$.

There is one more concept that will be of interest to us. If the motion of the $\mathrm{s}$ particles can be considered statistically independent, we have

$$
F_{s}\left(x_{1}, \ldots x_{s}\right)=\prod_{i=1}^{s} F_{1}\left(x_{i}\right)
$$

In this report we shall suppress the time variable $t$ when it is not important to the relation considered. We shall refer to motion for which the property (1) holds as uncorrelated motion. Motion for which (1) does not hold will be referred to as correlated motion.

The distribution functions have final interpretation when used to evaluate the average or expectation value of some mechanical property $A_{S}$ that depends on $s$ particles. We interpret this property as

$$
\left\langle A_{S}\right\rangle=\frac{1}{V^{s}} \int A_{S}\left(x_{1}, \ldots x_{s}\right) F_{s}\left(x_{1}, \ldots x_{s}\right) d x_{1} \ldots d_{s}
$$

where we have used the fact that the distribution functions are normalized as $\frac{1}{V} \int F_{1}\left(x_{1}\right) d x_{1}=1$. Obviously, we are primarily interested in $s=1$ and $s=2$.

Upon introducing the distribution functions, we are interested in their equations of motion rather than those of individual particles, although the two are closely related. One of the first successes in this direction was Boltzmann's equation for the one-particle distribution function for dilute gas of short-range potential.

$$
\begin{aligned}
& \frac{\partial \mathrm{F}_{1}\left(\mathrm{x}_{1}\right)}{\partial \mathrm{t}}+\overrightarrow{\mathrm{v}}_{1} \cdot \frac{\partial \mathrm{F}_{1}\left(\mathrm{x}_{1}\right)}{\partial \overrightarrow{\mathrm{q}}_{1}} \\
& \quad=\mathrm{n} \int_{0}^{2 \pi} \int_{0}^{\infty} \int_{\left(\mathrm{p}_{2}\right)}\left|\overrightarrow{\mathrm{v}_{2}-\mathrm{v}_{1}}\right|\left\{\mathrm{F}_{1}\left(\overrightarrow{\mathrm{p}}_{1}^{*}, \overrightarrow{\mathrm{q}}_{1}\right) \mathrm{F}_{1}\left(\overrightarrow{\mathrm{p}}_{2}^{*}, \overrightarrow{\mathrm{q}}_{2}\right)-\mathrm{F}_{1}\left(\overrightarrow{\mathrm{p}}_{1}, \overrightarrow{\mathrm{q}}_{2}\right) \mathrm{F}_{1}\left(\overrightarrow{\mathrm{p}}_{2}, \overrightarrow{\mathrm{q}}_{2}\right)\right\} \mathrm{d} \overrightarrow{\mathrm{p}}_{2} \text { adad } \phi .
\end{aligned}
$$

Here, $a$ is the collision impact parameter and $\vec{p}_{1}^{*}$ and $\vec{p}_{2}^{*}$ are the momenta that the par ticles must have had before the collision, given that their coordinates are now $x_{1}$ and $\mathbf{x}_{2}$. We shall consider no external force:

It may be recalled that Boltzmann's derivation depended upon a long free path between relatively quick collisions. As an approach to the problem of plasmas, this equation with a modified Coulomb potential was used by Spitzer and Härm. ${ }^{2}$ The collision integral with a straight $r^{-2}$-force diverges at long distances. Using the known fact that at equilibrium the effective interaction is the Debye potential, they cut the integral off at $\lambda_{D}$. Another approach was originally proposed by Vlasov ${ }^{3}$ and solved in detail by Landau. ${ }^{4}$ This emphasized the Coulomb nature of the interactions by considering the force on a 
charge to be given by the gradient of a potential whose source is $\int F_{1}\left(x_{1}\right) d p_{1}$. The equation for $\mathrm{F}_{1}$ becomes

$$
\frac{\partial \mathrm{F}_{1}\left(\mathrm{x}_{1}\right)}{\partial \mathrm{t}}+\overrightarrow{\mathrm{v}}_{1} \cdot \frac{\partial \mathrm{F}_{1}\left(\mathrm{x}_{1}\right)}{\partial \overrightarrow{\mathrm{q}}_{1}}=\mathrm{n} \frac{\mathrm{e}^{2}}{\mathrm{~m}} \frac{\partial \mathrm{F}_{1}\left(\mathrm{x}_{1}\right)}{\partial \overrightarrow{\mathrm{v}}_{1}} \cdot \frac{\partial}{\partial \overrightarrow{\mathrm{q}}_{1}} \int \frac{\mathrm{F}_{1}\left(\mathrm{x}_{2}\right) \mathrm{dx}}{\left|\overline{\mathrm{q}}_{1}-\mathrm{q}_{2}\right|} .
$$

As we shall see later, this equation is a first step but does not include or account for particle correlations.

A third approach to the problem may be made through a Fokker-Planck type of equation for $\mathrm{F}_{1}, 5$

$$
\frac{\partial F_{1}\left(x_{1}\right)}{\partial t}+V_{1} \cdot \frac{\partial F_{1}\left(x_{1}\right)}{\partial \vec{q}_{1}}=\frac{\partial}{\partial \vec{V}} \cdot\left(\vec{D}^{O} F_{1}\left(x_{1}\right)+\vec{B}^{O} \cdot \frac{\partial F_{1}\left(x_{1}\right)}{\partial \vec{V}_{1}}\right),
$$

where $\overrightarrow{D^{\circ}}$ and $\overrightarrow{B^{\circ}}$ are functionals of $F_{1}$. The form of this equation is derived by assuming that the particles undergo a large number of small deflections - a condition violated by Coulomb particles undergoing a close collision with large momentum transfer.

Before the Fokker-Planck equation can be of use, we must obtain the coefficients from considerations of the interactions. One method, described by Allis, ${ }^{6}$ is to expand the Boltzmann collision integral in terms of small deflections and obtain the FokkerPlanck form of equation. This, however, necessitates using the collision integral in the region in which its accuracy is most suspect - long-range interactions or grazing collisions. Another method described later ${ }^{7-11}$ uses the two-body distribution function under the assumption that the two bodies never get close together or experience an interaction that is strong compared with their kinetic energy. As discussed by Balescu, ${ }^{7}$ the coefficients of this equation have great intuitive appeal. However, integrals in them diverge at short distances because of a violation of the initial assumption; in this report we shall resolve this divergence.

One more fact should be noticed about these three equations. On the right-hand side of the Boltzmann, Vlasov, and Fokker-Planck equations we have functionals of $F_{1}$ only. The future of $\mathrm{F}_{1}$ is determined by its present value, but not obviously; we might, for example, find that the equations for $\mathrm{F}_{1}, \mathrm{~F}_{2}$, and so forth, are all interrelated in a set of simultaneous equations. As it is, the equation for $\mathrm{F}_{2}$ and the higher-order distribution functions must, in some sense, be trivial in the time variable so that they can be solved immediately with the result of a single equation for $F_{1}$. This occurrence will be discussed in great detail in section 2.1 .

The purpose of this report is to derive a kinetic equation for $F_{1}$. We shall derive this equation by starting with the general Liouville equation for the plasma and then examining the circumstances under which a kinetic equation for $\mathrm{F}_{1}$ can be assumed to exist. For these circumstances we shall obtain an equation for $F_{1}$ to first order in the small parameter $\left(e^{2} / k T \lambda_{D}\right)$. This will be an improvement upon the attempts mentioned 
above in the sense that it will contain no divergences in those terms corresponding to interactions with other particles, that is, the right-hand sides of $(3)-(5)$.

\subsection{STATEMENT OF THE PROBLEM}

Several authors ${ }^{8-10}$ have used the Liouville equation for the distribution function in the phase space of $6-\mathrm{N}$ dimensions as a starting point for the discussion of the evolution of a gas of $\mathrm{N}$ particles in a volume $\mathrm{V}$. This distribution function, $\mathrm{D}_{\mathrm{N}}\left(\mathrm{x}_{1}, \ldots \mathrm{x}_{\mathrm{S}} ; \mathrm{t}\right)$, is assumed to be symmetric under the interchange of any pair of particles. The Liouville equation is then

$$
\frac{\partial \mathrm{D}_{\mathrm{N}}}{\partial \mathrm{t}}=\left[\mathrm{H}_{\mathrm{N}}^{\mathrm{o}}+\sum_{\mathrm{i}<\mathrm{j}}^{\mathrm{N}} \psi_{\mathrm{ij}} ; \mathrm{D}_{\mathrm{N}}\right] .
$$

Here, the brackets are Poisson brackets,

$$
[F, G] \equiv \sum_{i=1}^{N}\left\{\frac{\partial F}{\partial \vec{q}_{i}} \cdot \frac{\partial G}{\partial \vec{p}_{i}}-\frac{\partial F}{\partial \vec{p}_{i}} \cdot \frac{\partial G}{\partial \bar{q}_{i}}\right\},
$$

and the kinetic and potential energies are

$$
\mathrm{H}_{\mathrm{N}}^{\mathrm{o}} \equiv \sum_{i=1}^{\mathrm{N}} \frac{\mathrm{p}_{\mathrm{i}}^{2}}{2 \mathrm{~m}}
$$

and

$$
\psi_{i j} \equiv \frac{e^{2}}{\left|\overrightarrow{q_{i}-q_{j}}\right|} .
$$

For our purposes throughout this report we shall assume only Coulomb interactions and identical electrons of mass $\mathrm{m}$ and charge $\mathrm{e}$ imbedded in a uniform background of opposite charge. The net charge will be taken to be neutral. The points to be investigated may be studied with this idealized model, without the complication of different particles.

We define the reduced distribution function for $s$ particles

$$
F_{S}\left(x_{1}, \ldots x_{S} ; t\right) \equiv \frac{1}{V^{N-s}} \int D_{N} d x_{S+1} \cdots d_{N}
$$

where the factor $\mathrm{V}^{\mathrm{N}-\mathrm{S}}$ is introduced to allow a transition to infinite volume. We shall also have use for the following identities: 


$$
\begin{aligned}
& \frac{1}{v^{N-S}} \int \frac{\partial D_{N}}{\partial t} d x_{s+1} \cdots d x_{N} \equiv \frac{\partial F_{S}}{\partial t}, \\
& \frac{1}{\mathrm{~V}^{\mathrm{N}-\mathrm{S}}} \int\left[\mathrm{H}_{\mathrm{N}}^{\mathrm{o}} ; \mathrm{D}_{\mathrm{N}}\right] \mathrm{dx}{ }_{\mathrm{S}+1} \cdots \mathrm{dx}_{\mathrm{N}} \equiv\left[\mathrm{H}_{\mathrm{S}}^{\mathrm{o}} ; \mathrm{F} \mathrm{s}\right] \\
& \frac{1}{V^{N-S}} \int\left[\sum_{i<j}^{N} \psi_{i j} ; D_{N}\right] d x_{S+1} \cdots d x_{N} \\
& \equiv\left[\sum_{i<j}^{S} \psi_{i j} ; F_{S}\right]+\frac{1}{V^{N-S}} \sum_{j=s+1}^{N} \sum_{i=1}^{S} \int\left[\psi_{i j} ; D_{N}\right] d x_{s+1} \ldots d_{N} \cdot
\end{aligned}
$$

By using the symmetry of $D_{N}$ under interchange of particles, the sum over $j$ consists of identical terms so that the last identity may be written

$$
\begin{aligned}
& \frac{1}{\mathrm{v}^{\mathrm{N}-\mathrm{S}}} \int\left[\sum_{i<j}^{\mathrm{N}} \psi_{i j} ; \mathrm{D}_{\mathrm{N}}\right] \mathrm{dx_{S+1 }} \ldots \mathrm{dx}_{\mathrm{N}} \\
& \equiv\left[\sum_{i<j}^{\mathrm{S}} \psi_{i j} ; \mathrm{F}_{\mathrm{S}}\right]+\frac{\mathrm{N}-\mathrm{s}}{\mathrm{V}} \int\left[\sum_{i=1}^{\mathrm{S}} \psi_{i s+1} ; \mathrm{F}_{\mathrm{s}+1}\right] \mathrm{dx_{s+1 }} .
\end{aligned}
$$

Integration of (6) over $\mathrm{dx}_{\mathrm{S}+1} \ldots \mathrm{dx}_{\mathrm{N}}$ and use of (9) produce

$$
\frac{\partial F_{s}}{\partial t}=\left[H_{s}^{O} ; F_{S}\right]+\left[\sum_{i<j}^{S} \psi_{i j} ; F_{S}\right]+\frac{N-s}{V} \int\left[\sum_{i=1}^{S} \psi_{i s+1} ; F_{S+1}\right] d x_{s+1} .
$$

Throughout this report the argument of the function $F_{S}$ is suppressed when the meaning is clear.

For fixed $s$ we may pass to the limit of infinite volume and infinite number so that $\frac{N}{V}$ remains constant. This replaces $\frac{N-S}{V}$ with $n$. For spacially nonuniform plasmas, $\mathrm{n}$ is not a density in quite the usual sense, but is the limit $\frac{\mathrm{N}}{\mathrm{V}}$.

The introduction of $\mathrm{n}$ gives (11) its final form. The meanings of its terms are clear. The left-hand side and the first two terms on the right-hand side constitute the Liouville equation including interactions for the $s$ particles under consideration. The integral term represents the contribution to the rate of change of $F_{S}$ which is due to the interactions with the rest of the particles.

In order to approach the analysis of the equations (11), we shall estimate the size of the terms. It will be found that under certain circumstances one term is small and thus gives rise to the possibility of a perturbation expansion. We assume a plasma that is near enough to equilibrium that we may define a shielding distance $\lambda_{D}=\sqrt{k T / 4 \pi n e^{2}}$, a plasma frequency $\omega_{p}=\sqrt{4 \pi n e^{2} / \mathrm{m}}$, and a characteristic velocity $\bar{v}=\sqrt{\mathrm{kT} / \mathrm{m}}$. By using 
these as units, the sizes of the various integrals and derivatives can be estimated. The change of coordinates $\tau=\omega_{p} t, p=r / \lambda_{D}$, and $u=v / \bar{v}$ in (11) will make, for the average particle, each of the integrals and derivatives of order one. The size of each term will be given by the following coefficients

$$
\omega_{p}, \quad \bar{v} / \lambda_{D}, \quad e^{2} / m \bar{v} b^{2}, \quad n e^{2} \lambda_{D} / m \bar{v}
$$

which are the ratios

$$
1, \quad 1,1 / \mathrm{n}_{\mathrm{D}} \mathrm{b}^{2}, \quad 1 .
$$

Here, $\mathrm{b}$ is the distance between the two particles under consideration. The last three terms are the three sums in (11), and the coefficients above simply represent their magnitudes. In particular, in the third term there will be a different $b$ for the separation of each pair of particles; the term for each pair is subject to the analysis given below.

A requirement that the third term be small implies that $b \gg \sqrt{e^{2} \lambda_{D} / k T}$. The solution of (11) has been discussed by several authors ${ }^{8-10}$ for the situations that satisfy this requirement for all pairs. The process has been to assume that all of the s-particle interaction terms are small and to assign to them an expansion parameter $g$ that is later set to one. The terms designated by $g^{r}$ will be of order $\left(e^{2} / k T \lambda_{D}\right)^{r}$. Then $F_{S}$ is expanded in $g$ as a perturbation expansion. This results in zero and first order in $\mathrm{g}$.

$$
\frac{\partial F_{s}^{o}}{\partial t}=\left[H_{S}^{o} ; F_{S}^{o}\right]+n \int\left[\sum_{i=1}^{S} \psi_{i s+1} ; F_{S+1}^{o}\right] d x_{S+1}
$$

and

$$
\frac{\partial F_{S}^{1}}{\partial t}=\left[H_{S}^{o} ; F_{S}^{l}\right]+\left[\sum_{i<j}^{S} \psi_{i j} ; F_{S}^{o}\right]+n \int\left[\sum_{i=1}^{S} \psi_{i s+1} ; F_{S+1}^{l}\right] d x_{s+1} .
$$

The derivation and solution of these equations will be discussed; of more interest to us now is the general form of the solution. We shall see that for a uniform plasma $\mathrm{F}_{\mathrm{S}}^{\mathrm{O}}$ and $\mathrm{F}_{\mathrm{S}}^{1}$ may be reduced to

$$
\begin{aligned}
& F_{s}^{o}=\prod_{i=1}^{S} F_{1}\left(\vec{p}_{i}\right) \\
& F_{S}^{1}=\sum_{i<j}^{S} \prod_{k \neq i, j}^{S} F_{1}\left(\vec{p}_{k}\right) F_{2}^{l}\left(\vec{p}_{i}, \vec{p}_{j},{\overline{q_{i}}-\bar{q}_{j}}\right) .
\end{aligned}
$$

Here, $F_{2}^{1}$ is the solution of the equation 


$$
\begin{aligned}
\frac{\partial \mathrm{F}_{2}^{1}\left(\mathrm{x}_{1}, \mathrm{x}_{2}\right)}{\partial \mathrm{t}}= & {\left[\mathrm{H}_{2}^{\mathrm{O}} ; \mathrm{F}_{2}^{1}\left(\mathrm{x}_{1}, \mathrm{x}_{2}\right)\right]+\left[\psi_{12} ; \mathrm{F}_{1}\left(\overline{\mathrm{p}}_{1}\right) \mathrm{F}_{1}\left(\overline{\mathrm{p}}_{2}\right)\right] } \\
& +\mathrm{n} \int\left\{\left[\psi_{13} ; \mathrm{F}_{1}\left(\overline{\mathrm{p}}_{1}\right) \mathrm{F}_{2}^{1}\left(\mathrm{x}_{2}, \mathrm{x}_{3}\right)\right]+\left[\psi_{23} ; \mathrm{F}_{1}\left(\overline{\mathrm{p}}_{2}\right) \mathrm{F}_{2}^{1}\left(\mathrm{x}_{1}, \mathrm{x}_{3}\right)\right]\right\} \mathrm{dx_{3 } ,}
\end{aligned}
$$

and to first order in $\mathrm{g}$

$$
\frac{\partial F_{1}\left(\bar{p}_{1}\right)}{\partial t}=n \int\left[\psi_{12} ; F_{2}^{1}\left(x_{1}, x_{2}\right)\right] d x_{2} \text {. }
$$

Equation 15 has been solved under the adiabatic hypothesis defined in section 2.1. When this solution is substituted in (16), the final equation becomes

$$
\begin{aligned}
& \frac{\partial \mathrm{F}_{1}\left(\mathrm{p}_{1}\right)}{\partial \mathrm{t}}=\mathrm{n} \int\left[\psi_{12} ; \mathrm{F}_{2}^{1}\left(\mathrm{x}_{1}, \mathrm{x}_{2}\right)\right] \mathrm{dx_{2 }} \cong \mathrm{n} \int\left[\psi_{12} ; \mathrm{f}_{2}^{1}\left(\mathrm{x}_{1}, \mathrm{x}_{2}\right)\right] \mathrm{dx} \mathrm{x}_{2}
\end{aligned}
$$

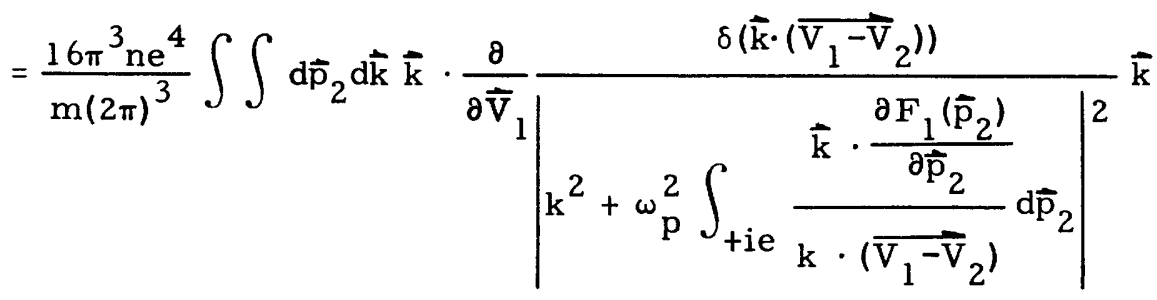

$$
\begin{aligned}
& \cdot\left(\frac{\partial}{\partial \overrightarrow{\mathrm{V}}_{1}}-\frac{\partial}{\partial \overrightarrow{\mathrm{V}}_{2}}\right) \mathrm{F}_{1}\left(\overline{\mathrm{p}}_{1}\right) \mathrm{F}_{1}\left(\overrightarrow{\mathrm{p}}_{2}\right)
\end{aligned}
$$

where, under the adiabatic hypothesis,

$$
F_{2}^{1}\left(x_{1}, x_{2}\right) \equiv f_{2}^{1}\left(x_{1}, x_{2}\right) .
$$

For definiteness, we reserve a special symbol for this function and refer to it as the large-separation solution.

In (17) $\vec{k}$ is the Fourier -transform variable corresponding to $\left(\overrightarrow{r_{1}-r_{2}}\right)$. This integration diverges logarithmically for large values of $|\vec{k}|$ corresponding to small values of $\left|\bar{r}_{1}-\bar{r}_{2}\right|$. As mentioned previously, this divergence occurs because we assumed that $\psi_{12}$ is small in the derivation of (15). The divergence occurs in a region that violates this assumption. To remove the divergence, one should allow for the possible mutual approach of particles 1 and 2 in (11).

In the classical gas of electrons considered, one never encounters the problem of two particles close together because of the mutual repulsions. This fact should be borne out in the solution of the set of equations (11). If discrete positive charges had been included, real problems might have occurred because of the attractive potential. The occurrence of bound states and the effects of very fast electrons would require analysis that would go far beyond the techniques employed here. In this investigation we shall study the effect of close collisions on the two-body distribution function and shall thereby 
remove divergence in the classical problem of Coulomb repulsions.

In the past this divergence has been handled by cutting off the integration in (17) at $\mathrm{kT} / \mathrm{e}^{2}$, the value corresponding to the distance of average closest approach. Since the dependence on the cutoff is logarithmic, the final results are not expected to be much in error from a numerical point of view. However, it is of interest to see what happens to the distribution function for close collisions and to see how accurate the method of cutting off may be.

In order to proceed let us return to the arguments that led to the assignments of orders of magnitude to the various terms in (11). We found that the requirement that the pair interaction term be small implied that the $s$ particles are mutually separated by distances $b \gg \sqrt{\mathrm{e}^{2} \lambda{ }_{\mathrm{D}} / \mathrm{kT}}$. Let us now imagine a set of concentric spheres, of possible separation of two particles, with radii $e^{2} / k T, \sqrt{e^{2} \lambda_{D} / k T}$, and $\lambda_{D}$. The radii are in the constant ratio $\sqrt{\mathrm{kT} \lambda_{\mathrm{D}} / \mathrm{e}^{2}}=\sqrt{4 \pi \mathrm{n} \lambda_{\mathrm{D}}^{3}}$. For a wide range of plasmas the quantity $4 \pi n \lambda_{\mathrm{D}}^{3}$ is much larger than one. For example, if $\mathrm{kT}=100 \mathrm{ev}$ and $\mathrm{n}=10^{16} \mathrm{~cm}^{-3}$, then $4 \pi n \lambda_{D}^{3} \sim 10^{5}$. These spheres are quite distinct, and they are useful in visualizing the process of interaction.

Let us label the spheres I, II, and III in order of increasing radii. The volume inside sphere I may be considered forbidden to the particles because $e^{2} / \mathrm{kT}$ is the distance of closest approach for the particle of average energy kT. Particles are allowed between spheres I and II; but in this region the potential energy is larger than the average kinetic energy, and thus the pair interaction term may not be considered small. The solution may be considered correct from sphere II outward, and an evaluation of (17) shows that the result is exponentially cut off outside $\lambda_{\mathrm{D}}$, in agreement with the Debye theory. In order to correctly handle the integral occurring in the equation for $F_{1}$, we must consider the possibility of particles occurring between spheres I and II.

Consider the number $n\left(e^{2} \lambda_{D} / k T\right)^{3 / 2}$, the probable number of particles inside sphere II. In terms of $4 \pi n \lambda_{D}^{3}$ this number is $\left(n \lambda_{D}^{3}\right)^{-1 / 2}$, which is small. We may argue from a strictly probabilistic point of view that it would be correspondingly even more unlikely that more particles should be inside this sphere. We are led to the concept of a "close collision," one in which two particles experience a short time interaction within sphere II. In line with the foregoing argument, we shall assume that the close collisions are binary and shall ignore the possibility of three particles occurring within this short range.

The analogy with the Boltzmann gas should be mentioned. For the Boltzmann gas we consider free particles undergoing binary collisions. For the Coulomb case we realize that the particles interact over a long range, but we use the fact that the strong inter-. actions occur only in binary types of events. In both cases those collisions that cause a large change in momentum are assumed to be binary.

We shall carry out the solution to (11) under the assumption that the interaction potential of one pair of particles, 1 and 2, is not necessarily small, while all other pairs are assumed to be small. The Hamiltonian to zero order for $s$ particles, including 1 and 2 , 
will be $\mathrm{H}_{\mathrm{S}}^{\mathrm{O}}+\Psi_{12}$. Sets of particles not including 1 and 2 will be assumed to be outside a range corresponding to a close collision; their zero-order Hamiltonian will be $\mathrm{H}_{\mathrm{S}}^{\mathrm{O}}$, and their solutions will be assumed to be (14)-(17). Whereas earlier treatments have assumed a gas of electrons experiencing entirely grazing collisions, we assume that one pair really collides with no limitations. This assumption is not as restricted as it sounds, since we are in reality saying that there are many mutually separated pairs in close collision.

This entire procedure will not get rid of all divergences, since in the equation for the two-body distribution function we encounter terms of the form

$$
\int\left[\psi_{13} ; F_{3}\left(x_{1}, x_{2}, x_{3}\right)\right] d x_{3}
$$

By the above-given procedure we correctly allow for $\psi_{12}$, but not the approach of 1 and 3 - the combination would entail a three-body collision. In section 3.2 it will be seen that this divergence can be circumvented in a plausible way. However, it is reasonable to expect that, if we kept this integral and merely cut it off, the dependence on the cutoff in the final equation for $F_{1}$ would be much weaker than the logarithmic dependence found in (17) because we have carried the problem to one more step of accuracy.

Section 2.1 will be devoted to a discussion of the methods, operators, and notation to be used in solving these equations. Section 2.2 will include, as an example, some discussion of the equations for the large-separation solution. In section 2.3 we shall modify the ideas of section 2.2 so that they will be applicable to the present problem. Section III will be a discussion of the actual problem, and Section IV will be a discussion of the results. Some of the material contained in section 2.1 is taken from a book by Bogoliubov ${ }^{12}$ but is included here since it is not usually used. 


\subsection{THE ADIABATIC HYPOTHESIS AND ITS IMPLICATIONS}

In order to study the methods to be used in the solution, introduce the Hamiltonian for the kinetic energies and mutual interactions of $s$ particles.

$$
H_{s}=H_{S}^{o}+\sum_{i<j}^{S} \psi_{i j}
$$

For simplicity, we assume that there is no external field. The generalization is conceptual immediately, since these are one-particle processes. However, the resulting particle trajectories are very difficult to solve. One would not expect that the evolutions of correlations are much affected by the presence of weak external fields; thus this model is useful intuitively for the more difficult case. The strong field, in which the external field exerts more force than most of the interactions, would be complicated. But for this simple case even the most simple equation, the Vlasov equation (4), is not understood, since its nonlinear character becomes important.

For the whole system the Liouville equation,

$$
\frac{\partial \mathrm{D}_{\mathrm{N}}}{\partial \mathrm{t}}=\left[\mathrm{H}_{\mathrm{N}} ; \mathrm{D}_{\mathrm{N}}\right]
$$

has as a formal solution

$$
D_{N}\left(x_{1}, \ldots x_{N} ; t\right)=S_{-t}^{N} D_{N}\left(x_{1}, \ldots x_{N} ; 0\right) \text {. }
$$

Here, the operator $S_{-t}^{N}$ operates on the particle coordinates $x_{1} \ldots x_{N}$ and projects them backward in time $t$ seconds on the basis of the paths given by their Hamiltonian; that is, $D_{N}$ flows like an ideal fluid in phase space.

Note the identity for an arbitrary $\phi$ :

$\frac{\partial}{\partial t} S_{-t}^{N} \phi\left(x_{1}, \ldots x_{N}, t\right)=\left[H_{N}, S_{-t}^{N} \phi\left(x_{1}, \ldots x_{N}, t\right)\right]+S_{-t}^{N} \frac{\partial}{\partial t} \phi\left(x_{1}, \ldots x_{N}, t\right)$.

This follows from the definition of $S_{-t}^{N}$ and the fact that

$$
\frac{\partial}{\partial t} S_{-\tau}^{N} \phi=\left(\frac{\partial S_{-t}^{N}}{\partial t}\right) \phi+S_{-t}^{N} \frac{\partial \phi}{\partial t}
$$

Another property that we shall need in this investigation is the solution of equations of the form

$$
\frac{\partial}{\partial t} \phi\left(x_{1}, \ldots x_{N} ; t\right)=\left[H_{N} ; \phi\left(x_{1}, \ldots x_{N} ; t\right)\right]+f\left(x_{1}, \ldots x_{N} ; t\right)
$$

Let $\phi\left(x_{1}, \ldots x_{N} ; t\right) \equiv S_{-t}^{N} x\left(x_{1}, \ldots x_{N} ; t\right)$; then, using (23) and (24), we obtain 


$$
S_{-t}^{N} \frac{\partial}{\partial t} x\left(x_{1}, \ldots x_{N} ; t\right)=f\left(x_{1}, x_{2} \ldots x_{N}, t\right) \text {. }
$$

Multiplying by $\mathrm{S}_{t}^{\mathrm{N}}$ and integrating, we obtain

$$
x\left(x_{1}, \ldots x_{N} ; t\right)-x\left(x_{1}, \ldots x_{N} ; 0\right)=\int_{0}^{t} S_{\tau}^{N_{f}}\left(x_{1}, \ldots x_{N} ; \tau\right) d \tau
$$

In (25) we use the relation

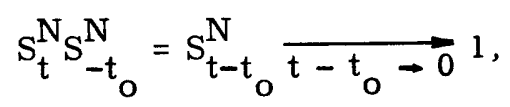

and consider it the inversion property of the operators. Finally, multiplying by $S_{-t}^{N}$ and resubstituting $\phi$, we have

$$
\phi\left(x_{1}, \ldots x_{N} ; t\right)=S_{-t}^{N} \phi\left(x_{1}, \ldots x_{N} ; 0\right)+\int_{0}^{t} d \tau S_{-(t-\tau)}^{N} f\left(x_{1}, \ldots x_{N} ; \tau\right) .
$$

In (26) the first term on the right-hand side is the contribution of flow in phase space and the second is the effect of the source.

Now let us examine the exact equation for $\mathrm{F}_{1}$,

$$
\frac{\partial \mathrm{F}_{1}\left(\mathrm{x}_{1}\right)}{\partial \mathrm{t}}=\left[\mathrm{H}_{1}^{\mathrm{o}} ; \mathrm{F}_{1}\left(\mathrm{x}_{1}\right)\right]+\mathrm{n} \int\left[\psi_{12} ; \mathrm{F}_{2}\left(\mathrm{x}_{1}, \mathrm{x}_{2}\right)\right] \mathrm{dx} \mathrm{x}_{2}
$$

This is obtained by integrating the Liouville equation and is equivalent to it. To use $\mathrm{F}_{2}$, we must solve the equation for $\mathrm{F}_{2}$, which involves knowing $\mathrm{F}_{3}$, and so forth. The advantage of this chain over the original Liouville equation is that, if we can break the chain in a physically sensible way, we can obtain a closed set of equations and know the precise approximations made in departing from the full Liouville equation. The problem is to perform the break in a manner that will balance the physical and computational reasonability.

Now look at (27) in the light of the fact that, as mentioned in section 1.1, the various forms of kinetic equations for $F_{1}$ have one thing in common - they can generally be written

$$
\frac{\partial F_{1}\left(x_{1}\right)}{\partial t}=A_{1}\left(x_{1} ; F_{1}\right) \text {. }
$$

Here, $A_{1}\left(x_{1} ; F_{1}\right)$ is a functional of $F_{1}$. The importance of this is that the entire time dependence of the right-hand side of (28) lies inside $F_{1}$ and depends only upon the current value of $F_{1}$. This fact is implied by having a kinetic equation for $F_{1}$ : that its present value is sufficient to predict its future.

If (27) is to be of the form of (28), then $F_{2}$ must be such that

$$
F_{2}\left(x_{1}, x_{2} ; t\right)=F_{2}\left(x_{1}, x_{2} ; F_{1}\right) \text {. }
$$

If (29) is to hold, it must be true for all $s \geqslant 2$ that 


$$
F_{S}\left(x_{1} \ldots x_{S} ; t\right)=F_{S}\left(x_{1} \ldots x_{S} ; F_{1}\right)
$$

Bogoliubov has shown ${ }^{12}$ that for a Boltzmann gas this is a very good assumption in that any initial $F_{S}$ that violates (30) will relax to the form (30) in a collision time that is very short compared with the characteristic time of change of $F_{1}$. Thus it is safe to assume that $\mathrm{F}_{\mathrm{S}}$ is of the form (30).

This whole argument breaks down for a plasma, particularly one that is not spacially uniform. In this case the collision time is of the order $\lambda_{D} / \bar{v}=\sqrt{m / 4 \pi n e^{2}}=\omega_{p}^{-1}$, which is the characteristic time of change of $F_{1}$ for a nonuniform plasma. However, to get a kinetic equation of the form (28), one is forced to take (30) as an assumption and to look for those solutions satisfying this form which will provide the most general equation of the form (28).

We begin our investigation with a generalization of the foregoing equations. We shall look for a pair of equations which is made up of (27) and an equation of the form

$$
\frac{\partial F_{2}\left(x_{1}, x_{2}\right)}{\partial t}=A_{2}\left(x_{1}, x_{2} ; F_{1}, F_{2}\right) \text {, }
$$

where the time dependence of $A_{2}$ resides within a functional dependence on $F_{1}$ and $F_{2}$. Since we know from (11) that

$$
\frac{\partial \mathrm{F}_{2}}{\partial \mathrm{t}}=\left[\mathrm{H}_{2}^{\mathrm{O}}+\psi_{12} ; \mathrm{F}_{2}\right]+\mathrm{n} \int\left[\psi_{13}+\psi_{23} ; \mathrm{F}_{3}\right] \mathrm{dx} \mathrm{x}_{3}
$$

we may say in analogy with (29) that

$$
F_{3}\left(x_{1}, x_{2}, x_{3} ; t\right)=F_{3}\left(x_{1}, x_{2}, x_{3} ; F_{1}, F_{2}\right) \text {, }
$$

and, therefore, that

$$
F_{s}\left(x_{1}, \ldots x_{s} ; t\right)=F_{s}\left(x_{1} \ldots x_{s} ; F_{1}, F_{2}\right)
$$

We shall refer to these functional-dependence assumptions as the "adiabatic hypotheses" in the following sense. For example, in (34), we assume that $F_{S}$ for $s>2$ relaxes very rapidly to a form depending only on the instantaneous values of $F_{1}$ and $F_{2}$. This assumption is analogous to the adiabatic approximation to the time-dependent perturbation theory in quantum mechanics.

This procedure serves the following purpose. We assume that a kinetic equation for $F_{2}$ exists, (31). Equation 31 implies certain limitations upon the time dependence of $F_{3}$, (33). We shall find the solution for $F_{3}$ which satisfies these limitations and use this function in the integral of (32) to obtain a general kinetic equation for $F_{2}$. We then have a method of investigating directly the possibilities and limitations of making another restriction on $F_{2}$, that is, that it is of the form $F_{2}\left(x_{1}, x_{2} ; F_{1}\right)$. We shall make this restriction and obtain a solution for $F_{2}$ in this form. Since the additional assumption that was made to obtain (17) from (15) is (29), this procedure will yield a result that is 
directly equivalent to (17) except that the divergence will no longer exist.

A look at the equations to be solved will show the origin of the adiabatic hypothesis and the ensuing statements. If the interaction $\psi_{12}$ is of order $\mathrm{g}$, a condition that we shall call Case I, we can write

$$
\frac{\partial F_{S}}{\partial t}=\left[H_{S}^{o} ; F_{S}\right]+g\left[\sum_{i<j}^{S} \psi_{i j} ; F_{S}\right]+n \int\left[\sum_{i=1}^{S} \psi_{i s+1} ; F_{S+1}\right] d x_{s+1} \text {. }
$$

When particles 1 and 2 are allowed to collide, $\psi_{12}$ is not small and must be included in the zero-order Hamiltonian. In this form we have Case II,

$$
\frac{\partial F_{S}}{\partial t}=\left[H_{S}^{o}+\psi_{12} ; F_{S}\right]+g\left[\sum_{i<j}^{S} \psi_{i j} ; F_{S}\right]+n \int\left[\sum_{i=1}^{s} \psi_{i s+1} ; F_{s+1}\right] d x_{s+1} \text {, }
$$

where the $\sum^{\prime}$ denotes the omission of the pair $\{12\}$.

We shall see that if $\mathrm{F}_{\mathrm{S}}$ is expanded in powers of $\mathrm{g}$,

$$
F_{s}=F_{s}^{0}+g F_{s}^{1}+g^{2} F_{s}^{2}+\ldots
$$

and a perturbation expansion is carried out, then the zero solutions are

CASE I:

$$
F_{S}^{o}=\prod_{i=1}^{S} F_{1}\left(x_{i}\right)
$$

and

\section{CASE II:}

$$
F_{s}^{o}=F_{2}\left(x_{1}, x_{2}\right) \prod_{i=3}^{S} F_{1}\left(x_{i}\right)
$$

In the perturbation expansions of (35) and (36), $F_{S}^{O}$ will serve as the source term for $F_{S}^{l}$ and we obtain

CASE I:

$$
\frac{\partial F_{S}^{1}}{\partial t}=\left[H_{s}^{o} ; F_{S}^{1}\right]+\left[\sum_{i<j}^{S} \psi_{i j} ; F_{S}^{o}\right]+n \int\left[\sum_{i=1}^{S} \psi_{i s+1} ; F_{S+1}^{1}\right] d x_{s+1}
$$

and

CASE II:

$$
\frac{\partial F_{s}^{1}}{\partial t}=\left[H_{S}^{o}+\psi_{12} ; F_{S}^{l}\right]+\left[\sum_{i<j}^{S} \psi_{i j} ; F_{S}^{o}\right]+n \int\left[\sum_{i=1}^{S} \psi_{i s+1} ; F_{s+1}^{1}\right] d x_{s+1} .
$$

With the adiabatic hypothesis applied to (39) we can study the time dependence of $F_{S}^{1}$ while 
holding $\mathrm{F}_{\mathrm{S}}^{\mathrm{O}}$ constant in the source. If, upon solution, $\mathrm{F}_{\mathrm{S}}^{\mathbf{l}}$ is found to vary as rapidly as $F_{S}^{O}$, then the hypothesis is invalid and another method is required.

The basis for the belief that for a uniform plasma the hypothesis is valid follows from the equation for $F_{1}$ in which we expand and use (38)

$$
\begin{aligned}
\frac{\partial F_{1}\left(x_{1}\right)}{\partial t}= & {\left[H_{1}^{o} ; F_{1}\left(x_{1}\right)\right]+n \int\left[\psi_{12} ; F_{1}\left(x_{1}\right) F_{1}\left(x_{2}\right)\right] d x_{2} } \\
& +g n \int\left[\psi_{12} ; F_{2}^{1}\left(x_{1}, x_{2}\right)\right] d x_{2}+g^{2} \ldots .
\end{aligned}
$$

The first integral is the effect of a potential $U$ whose source is $F_{1}$,

$$
\nabla^{2} U=-4 \pi n e^{2} \int F_{1}(x) d \vec{p}
$$

For the neutral uniform plasma, $U$ and the Poisson bracket of $F_{1}$ with $H_{1}^{O}$ are zero. The rate of change of $F_{1}$ is of order $g$; thus in equations like (39) we can hold $F_{S}^{O}$ constant to the same order in $\mathrm{g}$.

In this report we assume for Case $I$ and Case II that the $F_{S}$ depends functionally upon the functions appearing in $F_{S}^{O}$. In section 2.2 we work out Case I by assuming a dependence on $F_{1}$. This solution will serve as an introduction to the methods because this problem is closely related to the more general one. Section 2.3 and Section III will be concerned with Case II.

\subsection{THE LARGE-SEPARATION SOLUTION}

In this section we shall discuss the solution to those equations in which all twoparticle interactions are assumed to be small, which were referred to as Case I in section 2.1. We take (30) to be our form of the adiabatic hypothesis.

To carry out the solution, we must be able to handle terms of the form

$$
\frac{\partial}{\partial t} F_{s}\left(x_{1}, x_{2} \ldots x_{3} ; F_{1}\right) \text {, }
$$

for which we know that the derivative will operate only on the $F_{1}$, since that is the only place in which $t$ appears. For $s \geqslant 2$ expand $F_{s}$ in a power series in $g$, where $g$ is the expansion parameter described in section 1.2, to obtain (37). In (30) the expansion of $F_{2}$ will have the effect

$$
\frac{\partial F_{1}\left(x_{1}\right)}{\partial t}=\left[H_{1}^{o} ; F_{1}\left(x_{1}\right)\right]+n \int\left[\Psi_{12} ; F_{2}^{o}\left(x_{1}, x_{2} ; F_{1}\right)+g F_{2}^{1}\left(x_{1}, x_{2} ; F_{2}\right)+\ldots\right] d x_{2},
$$

and we shall consider this an expansion of $\partial \mathrm{F}_{1} / \partial \mathrm{t}$ in powers of $\mathrm{g}$. For convenience of notation we write (42)

$$
\frac{\partial F_{1}\left(x_{1}\right)}{\partial t}=A_{1}^{o}\left(x_{1} ; F_{1}\right)+g A_{1}^{l}\left(x_{1} ; F_{1}\right)+\ldots
$$


and define the $A_{1}^{r}$ by comparison with (43):

$$
\begin{aligned}
& A_{1}^{o}\left(x_{1} ; F_{1}\right)=\left[H_{1}^{o} ; F_{1}\left(x_{1}\right)\right]+n \int\left[\psi_{12} ; F_{2}^{o}\left(x_{1}, x_{2} ; F_{1}\right)\right] d x_{2}, \\
& A_{1}^{1}\left(x_{1} ; F_{1}\right)=n \int\left[\psi_{12} ; F_{2}^{1}\left(x_{1}, x_{2} ; F_{1}\right)\right] d x_{2},
\end{aligned}
$$

Let $x\left(x_{1}, \ldots x_{s} ; F_{1}\right)$ be any expression whose time dependence is completely defined by $\mathrm{F}_{1}$. For infinitesimal variations in $\mathrm{F}_{1}$,

$$
\delta x\left(x_{1}, x_{2} \ldots x_{s} ; F_{1}\right)=\phi\left(x_{1}, \ldots x_{S} ; F_{1}, \delta F_{1}\right),
$$

where $\phi$ is some new functional that will be linear in $\delta F_{1}$. From this relationship and (44), we have

$$
\begin{aligned}
\frac{\partial}{\partial t} x\left(x_{1}, \ldots x_{S} ; F_{1}\right) & =\phi\left(x_{1}, \ldots x_{S} ; F_{1} \frac{\partial F_{1}}{\partial t}\right) \\
& =\phi\left(x_{1}, \ldots x_{S} ; F_{1}, A^{o}\right)+g \phi\left(x_{1}, \ldots x_{S} ; F_{1}, A_{1}^{1}\right)+g^{2} \ldots
\end{aligned}
$$

or

$$
\frac{\partial x}{\partial t}=D_{0} x+g D_{1} x+g^{2} \cdots
$$

Here, $D_{r}$ denotes an operator that differentiates with respect to $t$ (by operating on $F_{1}$ ) and then replaces $\frac{\partial F_{1}}{\partial t}$ with $A_{1}^{r}$.

We wish to apply these definitions to the equation for $F_{S}$, Case $I$,

$$
\frac{\partial F_{S}}{\partial t}=\left[H_{S}^{o} ; F_{S}\right]+g\left[\sum_{i<j}^{S} \psi_{i j} ; F_{S}\right]+n \int\left[\sum_{i=1}^{s} \psi_{i s+1} ; F_{S+1}\right] d x_{s+1} \text {. }
$$

In (46) expand $\partial F_{S} / \partial t$ as in (45), and expand $F_{S}$ as in (37) to obtain

$$
\begin{aligned}
\left(D_{0}+g D_{1}+g^{2} \ldots\right)\left(F_{s}^{o}+g F_{s}^{1}+g^{2} F_{s}^{2}+\ldots\right)= & {\left[H_{s}^{o}+g \sum_{i<j}^{S} \psi_{i j} ; F_{s}^{o}+g F_{s}^{l}+g^{2} F_{s}^{2} \ldots\right] } \\
& +n \int\left[\sum_{i=1}^{S} \psi_{i s+1} ; F_{s+1}^{o}+g F_{s+1}^{1}+g^{2} F_{s+1}^{2} \ldots\right] d x_{s+1} .
\end{aligned}
$$

Using the first two powers of $g$, we obtain the following two equations: 


$$
\mathrm{D}_{\mathrm{o}} \mathrm{F}_{\mathrm{s}}^{\mathrm{o}}=\left[\mathrm{H}_{\mathrm{s}}^{\mathrm{o}} ; \mathrm{F}_{\mathrm{S}}^{\mathrm{o}}\right]+\mathrm{n} \int\left[\sum_{\mathrm{i}=1}^{\mathrm{s}} \psi_{\mathrm{is}+1} ; \mathrm{F}_{\mathrm{s}+1}^{\mathrm{o}}\right] \mathrm{dx} \mathrm{s}_{\mathrm{s}+1}
$$

and

$$
D_{O} F_{S}^{1}+D_{1} F_{S}^{o}=\left[H_{S}^{o} ; F_{S}^{1}\right]+\left[\sum_{i<j}^{S} \psi_{i j} ; F_{S}^{o}\right]+n \int\left[\sum_{i=1}^{S} \psi_{i s+1} ; F_{S+1}^{1}\right] d x_{s+1}
$$

Since in the limit of infinite separation of all $s$ particles the motions of the particles must become uncorrelated, we impose the following boundary conditions:

$$
\begin{aligned}
& F_{S}^{o} \underset{\text { all } \overline{q_{i}-q_{j} \rightarrow \infty}}{\longrightarrow} \prod_{i=1}^{S} F_{1}\left(x_{i}\right) \\
& \mathrm{F}_{\mathrm{s}}^{\mathrm{i}} \underset{\text { all }{\overline{q_{i}-\mathrm{q}_{j} \rightarrow \infty}}^{-}}{0} \quad \mathrm{i} \geqslant 1 .
\end{aligned}
$$

Here, $F_{S}^{i}$ goes to zero because $F_{S}^{o}$ is assumed to have all of the boundary contribution. By direct substitution and the use of the definition of the $D_{r}$ operators in terms of $A_{1}^{r}$, we see that the solution of (47) which satisfies $(38)$ is

$$
F_{S}^{o}\left(x_{1}, \ldots x_{S} ; F_{1}\right)=\prod_{i=1}^{S} F_{1}\left(x_{i}\right)
$$

In solving (48) we use the fact that, by definition of $D_{1}$,

$$
\begin{aligned}
D_{1} F_{S}^{o} & =D_{1} \prod_{i=1}^{S} F_{1}\left(x_{i}\right)=\sum_{i=1}^{S} \prod_{\substack{j=1 \\
\neq i}}^{S} F_{1}\left(x_{j}\right) A_{1}^{1}\left(x_{i} ; F_{1}\right) \\
& =n \sum_{i=1}^{S} \prod_{\substack{j=1 \\
\neq i}}^{S} F_{1}\left(x_{j}\right) \int\left[\psi_{i s+1} ; F_{2}^{1}\left(x_{i}, x_{s+1} ; F_{1}\right)\right] d x_{s+1} .
\end{aligned}
$$

Substitution of (51) in (48) yields

$$
\begin{aligned}
D_{o} F_{S}^{1}= & {\left[H_{S}^{o} ; F_{S}^{1}\right]+\left[\sum_{i<j}^{S} \psi_{i j} ; F_{S}^{o}\right]+n \int\left[\sum_{i=1}^{S} \psi_{i s+1} ; F_{s+1}^{1}\right] d x_{s+1} } \\
& -n \sum_{i=1}^{S} \prod_{\substack{j=1 \\
\neq i}}^{S} F_{1}\left(x_{j}\right) \int\left[\psi_{i s+1} ; F_{2}^{1}\left(x_{i}, x_{s+1}\right)\right] d x_{s+1} .
\end{aligned}
$$

We see that by straightfor ward substitution and application of (44) and (50), the solution of (52) which is consistent with the boundary condition (49) is 


$$
F_{S}^{1}=\sum_{1 \leqslant i<j \leqslant s} \prod_{\substack{k=1 \\ \neq i, j}}^{s} F_{1}\left(x_{k}\right) F_{2}^{l}\left(x_{i}, x_{j} ; F_{1}\right) .
$$

The final equation for $F_{2}^{l}\left(x_{1}, x_{2} ; F_{1}\right)$ is

$$
\begin{aligned}
\mathrm{D}_{\mathrm{o}} \mathrm{F}_{2}^{1}\left(\mathrm{x}_{1}, \mathrm{x}_{2} ; \mathrm{F}_{1}\right)= & {\left[\mathrm{H}_{2}^{\mathrm{o}} ; \mathrm{F}_{2}^{l}\left(\mathrm{x}_{1}, \mathrm{x}_{2} ; \mathrm{F}_{1}\right)\right]+\left[\psi_{12} ; \mathrm{F}_{1}\left(\mathrm{x}_{1}\right) \mathrm{F}_{1}\left(\mathrm{x}_{2}\right)\right] } \\
& +\mathrm{n} \int\left\{\left[\psi_{13} ; \mathrm{F}_{1}\left(\mathrm{x}_{1}\right) \mathrm{F}_{2}^{1}\left(\mathrm{x}_{2}, \mathrm{x}_{3} ; \mathrm{F}_{1}\right)\right]+\left[\psi_{23} ; \mathrm{F}_{1}\left(\mathrm{x}_{2}\right) \mathrm{F}_{2}^{1}\left(\mathrm{x}_{1}, \mathrm{x}_{3} ; \mathrm{F}_{1}\right)\right]\right\} \mathrm{d} \mathrm{x}_{3} \\
& +\mathrm{n} \int\left[\psi_{13}+\psi_{23} ; \mathrm{F}_{2}^{1}\left(\mathrm{x}_{1}, \mathrm{x}_{2} ; \mathrm{F}_{1}\right) \mathrm{F}_{1}\left(\mathrm{x}_{3}\right)\right] \mathrm{dx}_{3}
\end{aligned}
$$

For uniform plasmas, this equation has been solved by Lenard ${ }^{10}$ and Dupree. ${ }^{11}$ The latter's solution is carried out in the Appendix by using methods to be introduced in Section III. This solution results in the expression (17), which was solved under the adiabatic hypothesis. Thus in line with (18), the solution to (53) will be denoted $\mathrm{f}_{2}^{\mathrm{l}}\left(\mathrm{x}_{1}, \mathrm{x}_{2} ; \mathrm{F}_{1}\right)$.

\subsection{COLLISION PROBLEM ASSUMPTIONS}

In this section we discuss the equations referred to as Case II, (36), in which $\psi_{12}$ is not assumed to be of order $\mathrm{g}$ and the expansion parameter has been inserted before those terms taken to be small. (See section 1.2.)

By selecting particles 1 and 2 as the particular particles whose close approach will be allowed, we temporarily destroy the interchangeability of particles in $F_{S}$. Thus when we make an adiabatic hypothesis for $F_{S}(E q .34)$ and take $\partial F_{s} / \partial t$, we must interpret the effect of $\partial F_{1} / \partial t$ and $\partial F_{2} / \partial t$ differently, according to their arguments. Since in (36) we assumed that all $\psi_{i j}$ are small for $i$ and $j$ which are not equal to 1 and 2 , we expect the correlations between these pairs to be the same as those studied in section 2.2 because the correlation of a pair of particles is generated by the past history of their mutual force. Therefore, for these $i$ and $j$, we take $\partial F_{1}\left(x_{i}\right) / \partial t$ and $\partial F_{2}\left(x_{i}, x_{j}\right) / \partial t$ to be given by the results of section 2.2 .

In (10) we obtained the factor $\frac{\mathrm{N}-\mathrm{S}}{\mathrm{V}}$ before the integral by summing over identical particles. If in this new interpretation we are careful to sum over only the identical ones, we will have as an equation for $F_{1}\left(x_{i}\right)$

$$
\begin{aligned}
\frac{\partial F_{1}\left(x_{i}\right)}{\partial t}= & {\left[H_{1}^{o} ; F_{1}\left(x_{i}\right)\right]+\frac{N-3}{V} \int\left[\psi_{i s+1} ; F_{2}\left(x_{i}, x_{s+1}\right)\right] d x_{s+1} } \\
& +\frac{1}{V} \int\left[\psi_{i 1} ; F_{2}\left(x_{i}, x_{1}\right)\right] d x_{1}+\frac{1}{V} \int\left[\psi_{i 2} ; F_{2}\left(x_{i}, x_{2}\right)\right] d x_{2} .
\end{aligned}
$$

However, the last two integrals have small effect for two reasons. First, in the limit of large N, their contribution will become negligible. Second, we know that outside a 
range corresponding to a close collision all $\mathrm{F}_{2}$ are the same, so that to this extent they can be included in the first integral. We have said the $\psi_{i 1}$ or $\psi_{i 2}$ must be small, just as $\psi_{\text {is }+1}$ is small; thus the contribution must be essentially the same. A similar argument holds for the evolution of $F_{2}\left(x_{1}, x_{2}\right)$.

This distinction may be summarized in the following way. When we write an equation for $F_{S}$, the Hamiltonian will contain $\psi_{12}$ to zero order in $g$ only if the set $s$ contains both 1 and 2. We assume that, if this zero-order Hamiltonian does not contain $\Psi_{12}$, the evolution of $F_{S}$ is the same as that discussed in section 2.2. This assumption is connected with the binary collision assumption. We do not complicate the picture by including close collisions of mutually separated pairs and we explicitly exclude 3-body collisions .

With this in mind we are able to interpret differentiations of the form $\frac{\partial}{\partial t} F_{s}\left(x_{1}, \ldots x_{s}\right.$; $\left.F_{1}, F_{2}\right)$. When the derivative operates on $F_{1}\left(x_{i}\right)$ or $F_{2}\left(x_{i}, x_{j}\right)$, we apply $A_{1}^{r}$ defined in section 2.2. When the derivative operates on $F_{2}\left(x_{1}, x_{2}\right)$ we must define new $A_{2}$. By comparing (31) with the equation for $\mathrm{F}_{2}\left(\mathrm{x}_{1}, \mathrm{x}_{2}\right)$,

$$
\frac{\partial \mathrm{F}_{2}\left(\mathrm{x}_{1}, \mathrm{x}_{2}\right)}{\partial \mathrm{t}}=\left[\mathrm{H}_{2}^{\mathrm{o}}+\psi_{12} ; \mathrm{F}_{2}\left(\mathrm{x}_{1}, \mathrm{x}_{2}\right)\right]+\mathrm{n} \int\left[\psi_{13}+\psi_{23} ; \mathrm{F}_{3}\left(\mathrm{x}_{1}, \mathrm{x}_{2}, \mathrm{x}_{3} ; \mathrm{F}_{1}, \mathrm{~F}_{2}\right)\right] \mathrm{d} \mathrm{x}_{3},
$$

expanding in (54)

$$
F_{3}=F_{3}^{o}+g F_{3}^{1}+g^{2} F_{3}^{2}+\ldots
$$

and expanding in (31)

$$
A_{2}\left(x_{1}, x_{2} ; F_{1}, F_{2}\right)=A_{2}^{o}\left(x_{1}, x_{2} ; F_{1}, F_{2}\right)+g A_{2}^{l}\left(x_{1}, x_{2} ; F_{1}, F_{2}\right)+g^{2} \ldots
$$

we obtain

$$
\begin{aligned}
A_{2}^{o}\left(x_{1}, x_{2} ; F_{1}, F_{2}\right)= & {\left[H_{2}^{o}+\Psi_{12} ; F_{2}\left(x_{1}, x_{2}\right)\right] } \\
& +n \int\left[\Psi_{13}+\psi_{23} ; F_{3}^{o}\left(x_{1}, x_{2}, x_{3} ; F_{1}, F_{2}\right)\right] d x_{3} \\
A_{2}^{1}\left(x_{1}, x_{1} ; F_{1}, F_{2}\right)= & n \int\left[\Psi_{13}+\Psi_{23} ; F_{3}^{1}\left(x_{1}, x_{2}, x_{3} ; F_{1}, F_{2}\right)\right] d x_{3}
\end{aligned}
$$




\section{COLLISION PROBLEM SOLUTION}

In section 3.1 we shall use the results of section 2.3 in an equation for $\mathrm{F}_{3}^{\mathrm{l}}$. In section 3.2 we shall solve this equation and use it in a solution of the equation for $\mathrm{F}_{2}$.

\subsection{THE EQUATION FOR $\mathrm{F}_{3}^{1}$}

In order to derive an equation for $F_{3}^{l}\left(x_{1}, x_{2}, x_{3} ; F_{1}, F_{2}\right)$, we start with Eq. 36 in which we wish to obtain an expansion for $F_{3}$ which is analogous to (37). In (36) we no longer require that $\psi_{12}$ be of order $g$, and we assume that its time dependence occurs through a functional dependence on $F_{1}$ and $F_{2}$. Expand $\partial / \partial t$ as in (45), $F_{S}$ as in (37), and $F_{S+1}$; then

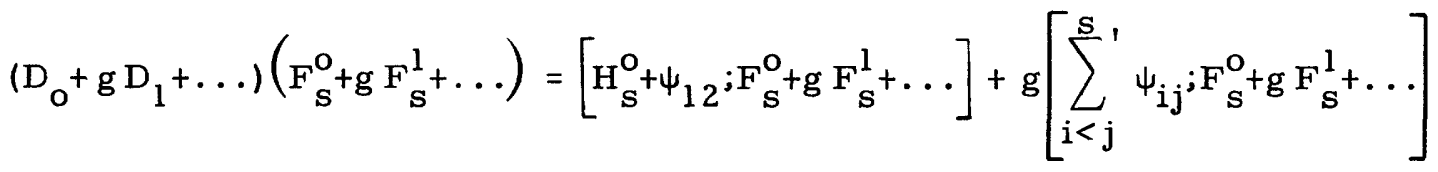

$$
\begin{aligned}
& +n \int\left[\sum_{i=1}^{s} \psi_{i s+1} ; F_{s+1}^{o}+g F_{s+1}^{1}+\ldots\right] d x_{s+1} \text {. }
\end{aligned}
$$

Equating the first two powers of $g$, we obtain

$$
\mathrm{D}_{\mathrm{o}} \mathrm{F}_{\mathrm{S}}^{\mathrm{o}}=\left[\mathrm{H}_{\mathrm{S}}^{\mathrm{O}}+\psi_{12} ; \mathrm{F}_{\mathrm{S}}^{\mathrm{o}}\right]+\mathrm{n} \int\left[\sum_{\mathrm{i}=1}^{\mathrm{S}} \psi_{\mathrm{is}+1} ; \mathrm{F}_{\mathrm{s}+1}^{\mathrm{o}}\right] \mathrm{dx} \mathrm{s}_{\mathrm{S}+1}
$$

and

$$
D_{o} F_{S}^{1}+D_{1} F_{S}^{o}=\left[H_{S}^{o}+\psi_{12} ; F_{S}^{1}\right]+\left[\sum_{i<j}^{S} \psi_{i j} ; F_{s}^{o}\right]+n \int\left[\sum_{i=1}^{s} \psi_{i s+1} ; F_{s+1}^{1}\right] d x_{s+1} \text {. }
$$

Since in the limit of infinite separation of all $\mathrm{s}$ particles except 1 and 2 the motions must become uncorrelated, we impose the following boundary conditions

$$
\begin{aligned}
& F_{s}^{o}\left(x_{1}, \ldots x_{s} ; F_{1}, F_{2}\right) \underset{\text { all }{\overline{q_{j}}-\bar{q}_{i} \rightarrow \infty}_{2}}{\longrightarrow} F_{2}\left(x_{1}, x_{2}\right) \prod_{i=3}^{S} F_{1}\left(x_{i}\right) \\
& \text { except } 12
\end{aligned}
$$

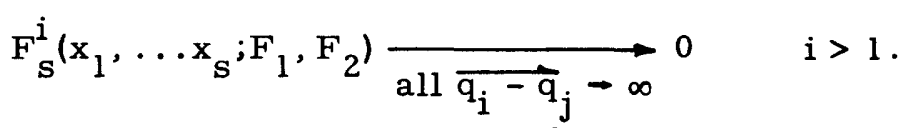

$$
\begin{aligned}
& \text { except } 12
\end{aligned}
$$

The boundary condition for $\mathrm{F}_{2}\left(\mathrm{x}_{1}, \mathrm{x}_{2}\right)$ will not be introduced here because it is not needed, since $F_{S}^{O}$ has $F_{2}\left(x_{1}, x_{2}\right)$ in it. The boundary conditions are used only to show the asymptotic form of $\mathrm{F}_{\mathrm{S}}^{\mathrm{O}}$.

The solution to (56) which matches these boundary conditions is 


$$
F_{s}^{o}\left(x_{1}, \ldots x_{s} ; F_{1}, F_{2}\right)=F_{2}\left(x_{1}, x_{2}\right) \prod_{i=3}^{S} F_{1}\left(x_{i}\right)
$$

Here, we have used the facts that

$$
\mathrm{D}_{\mathrm{o}} \mathrm{F}_{2}\left(\mathrm{x}_{1}, \mathrm{x}_{2}\right)=\mathrm{A}_{2}^{\mathrm{O}}\left(\mathrm{x}_{1}, \mathrm{x}_{2} ; \mathrm{F}_{1}, \mathrm{~F}_{2}\right)
$$

and

$$
D_{0} F_{1}\left(x_{i}\right)=A_{1}^{O}\left(x_{i} ; F_{1}\right)
$$

To solve (57), we must know the function $D_{1} F_{S}^{o}$. Using the section 2.2 prescription for $D_{1}$, we have

$$
\begin{aligned}
D_{1} F_{s}^{o}= & \prod_{i=3}^{s} F_{1}\left(x_{i}\right) D_{1} F_{2}\left(x_{1}, x_{2}\right)+F_{2}\left(x_{1}, x_{2}\right) \sum_{i=3}^{s} \prod_{\substack{j=3 \\
\neq i}}^{s} F_{1}\left(x_{j}\right) D_{1} F_{1}\left(x_{i}\right) \\
= & n \prod_{i=3}^{S} F_{1}\left(x_{i}\right) \int\left[\psi_{1 s+1}+\psi_{2 s+1} ; F_{3}^{l}\left(x_{1}, x_{2}, x_{s+1} ; F_{1}, F_{2}\right)\right] d x_{s+1} \\
& +n F_{2}\left(x_{1}, x_{2}\right) \sum_{i=3}^{s} \underset{\substack{j=3 \\
\neq i}}{s} F_{1}\left(x_{j}\right) \int\left[\psi_{i s+1} ; f_{2}^{l}\left(x_{i}, x_{s+1} ; F_{1}\right)\right] d x_{s+1} .
\end{aligned}
$$

The implications of the discussion in section 2.3 are used. Since the $i^{\text {th }}$ particle is assumed not to interact closely with the other particles, the function $f_{2}^{1}\left(x_{i}, x_{s+1}\right)$ in (59) is the large-separation function of section 2.3.

Using (59) in (57) we arrive at the following expression for $D_{0} F_{S}^{1}$ :

$$
\begin{aligned}
& D_{o} F_{S}^{l}\left(x_{1}, \ldots x_{s} ; F_{1}, F_{2}\right)=\left[H_{s}^{O}+\psi_{12} ; F_{S}^{l}\left(x_{1}, \ldots x_{s} ; F_{1}, F_{2}\right)\right]+\left[\sum_{i<j}^{S} \psi_{i j} ; F_{s}^{o}\left(x_{1}, \ldots x_{s} ; F_{1}, F_{2}\right)\right] \\
& +n \int\left[\sum_{i=1}^{s} \psi_{i s+1} ; F_{s+1}^{1}\left(x_{1} \ldots x_{s}+1 ; F_{1}, F_{2}\right)\right] d x_{s+1} \\
& -n \prod_{i=3}^{S} F_{1}\left(x_{i}\right) \int\left[\psi_{1 s+1}+\psi_{2 s+1} ; F_{3}^{1}\left(x_{1}, x_{2}, x_{s+1} ; F_{1}, F_{2}\right)\right] d x_{s+1} \\
& -n F_{2}\left(x_{1}, x_{2}\right) \sum_{i=3}^{S} \prod_{\substack{j=3 \\
\neq i}}^{S} F_{1}\left(x_{j}\right) \int\left[\psi_{i s+1} ; f_{2}^{1}\left(x_{i}, x_{s+1}\right)\right] d x_{s+1}
\end{aligned}
$$

By direct substitution the solution to $(60)$ is 


$$
\begin{aligned}
F_{S}^{l}= & F_{2}\left(x_{1}, x_{2}\right) \sum_{3 \leqslant i<j \leqslant s} \prod_{\substack{k=3 \\
k i, j}}^{s} F_{1}\left(x_{k}\right) f_{2}^{l}\left(x_{i}, x_{j}\right) \\
& +\sum_{i=3}^{S} \prod_{\substack{k=3 \\
\neq i}}^{s} F_{1}\left(x_{k}\right) F_{3}^{l}\left(x_{1}, x_{2}, x_{i} ; F_{1}, F_{2}\right) .
\end{aligned}
$$

Here, $F_{3}^{1}$ is the solution to

$$
\begin{aligned}
\mathrm{D}_{\mathrm{o}} \mathrm{F}_{3}^{1}\left(\mathrm{x}_{1}, \mathrm{x}_{2}, \mathrm{x}_{3} ; \mathrm{F}_{1}, \mathrm{~F}_{2}\right)= & {\left[\mathrm{H}_{3}^{\mathrm{o}}+\psi_{12} ; \mathrm{F}_{3}^{1}\left(\mathrm{x}_{1}, \mathrm{x}_{2}, \mathrm{x}_{3} ; \mathrm{F}_{1}, \mathrm{~F}_{2}\right)\right] } \\
& +\mathrm{n} \int\left[\psi_{14}+\psi_{24}+\psi_{34} ; \mathrm{F}_{1}\left(\mathrm{x}_{4}\right) \mathrm{F}_{3}^{1}\left(\mathrm{x}_{1}, \mathrm{x}_{2}, \mathrm{x}_{3} ; \mathrm{F}_{1}, \mathrm{~F}_{2}\right)\right] \mathrm{dx_{4 }} \\
& +\mathrm{n} \int\left[\psi_{34} ; \mathrm{F}_{1}\left(\mathrm{x}_{3}\right) \mathrm{F}_{3}^{1}\left(\mathrm{x}_{1}, \mathrm{x}_{2}, \mathrm{x}_{4} ; \mathrm{F}_{1}, \mathrm{~F}_{2}\right)\right] \mathrm{dx_{3 }} \\
& +\left[\psi_{13}+\psi_{23} ; \mathrm{F}_{2}\left(\mathrm{x}_{1}, \mathrm{x}_{2}\right) \mathrm{F}_{1}\left(\mathrm{x}_{3}\right)\right] \\
& +\mathrm{n} \int\left[\psi_{14}+\psi_{24} ; \mathrm{F}_{2}\left(\mathrm{x}_{1}, \mathrm{x}_{2}\right) \mathrm{f}_{2}^{1}\left(\mathrm{x}_{3}, \mathrm{x}_{4} ; \mathrm{F}_{1}\right)\right] \mathrm{dx_{4 }} .
\end{aligned}
$$

The first integral is zero for a uniform plasma, since it is the effect of the potential $U$ in (41).

For convenience we put (61) into another form and thus give an analytical meaning to $\mathrm{D}_{\mathrm{o}}$.

Since for the uniform plasma the integral terms in $A_{2}^{O}\left(x_{1}, x_{2}\right)$ and $A_{1}^{O}\left(x_{3}\right)$ are zero, we have

$$
\begin{aligned}
& \mathrm{D}_{\mathrm{o}} \mathrm{F}_{2}\left(\mathrm{x}_{1}, \mathrm{x}_{2}\right)=\left[\mathrm{H}_{2}^{\mathrm{O}}+\psi_{12} ; \mathrm{F}_{2}\left(\mathrm{x}_{1}, \mathrm{x}_{2}\right)\right] \\
& \mathrm{D}_{\mathrm{O}} \mathrm{F}_{1}\left(\mathrm{x}_{3}\right)=\left[\mathrm{H}_{1}^{\mathrm{O}} ; \mathrm{F}_{1}\left(\mathrm{x}_{3}\right)\right] .
\end{aligned}
$$

Now introduce the operator $S_{-\tau}^{1}$ that projects particle 3 backward $\tau$ seconds along a path given by the free-particle Hamiltonian. For any function $\phi\left(x_{3}\right)$,

$$
\frac{\partial}{\partial \tau} \mathrm{S}_{-\tau}^{1} \phi\left(\mathrm{x}_{3}\right)=\left[\mathrm{H}_{1}^{\mathrm{O}} ; \mathrm{S}_{-\tau}^{1} \phi\left(\mathrm{x}_{3}\right)\right] \text {. }
$$

Likewise introduce the operator $\mathrm{S}_{-\tau}^{2}$ that projects particles 1 and 2 backward along the paths given by their Hamiltonian including interactions. In operating on the coordinates of a colliding pair at $x_{1}$ and $x_{2}$, this operator will produce the coordinates $x_{1}(-\tau)$ and $x_{2}(-\tau)$. In analogy with (63), we have

$$
\frac{\partial}{\partial \tau} S_{-\tau}^{2} \phi\left(x_{1}, x_{2}\right)=\left[H_{2} ; S_{-\tau}^{2} \phi\left(x_{1}, x_{2}\right)\right] \text {. }
$$


Using $F_{1}\left(x_{3}\right)$ and $F_{2}\left(x_{1}, x_{2}\right)$ in (63) and (64), we have

$$
\begin{aligned}
& \frac{\partial}{\partial \tau} S_{-\tau}^{1} F_{1}\left(x_{3}\right)=\left[H_{1}^{o} ; S_{-\tau}^{1} F_{1}\left(x_{3}\right)\right] \\
& \frac{\partial}{\partial \tau} S_{-\tau}^{2} F_{2}\left(x_{1}, x_{2}\right)=\left[H_{2}^{O}+\psi_{12} ; S_{-\tau}^{2} F_{2}\left(x_{1}, x_{2}\right)\right] .
\end{aligned}
$$

Comparing (62) and (65), we have the identity

$$
\mathrm{D}_{\mathrm{o}} \mathrm{F}_{3}^{1}\left(\mathrm{x}_{1}, \mathrm{x}_{2}, \mathrm{x}_{3} ; \mathrm{S}_{-\tau}^{\mathrm{l}} \mathrm{F}_{1}, \mathrm{~S}_{-\tau}^{2} \mathrm{~F}_{2}\right)=\frac{\partial}{\partial \tau} \mathrm{F}_{3}^{1}\left(\mathrm{x}_{1}, \mathrm{x}_{2}, \mathrm{x}_{3} ; \mathrm{S}_{-\tau}^{1} \mathrm{~F}_{1}, \mathrm{~S}_{-\tau}^{2} \mathrm{~F}_{2}\right) \text {; }
$$

that is, if in the functional dependence we use $S_{-\tau}^{1} F_{1}$ and $S_{-\tau}^{2} F_{2}$ instead of $F_{1}$ and $F_{2}$, the $\mathrm{D}_{\mathrm{O}}$ operator can be replaced with $\partial / \partial \tau$ because the effect is the same. By using this relationship, (61) becomes

$$
\begin{aligned}
\frac{\partial}{\partial \tau} F_{3}^{l}\left(x_{1}, x_{2}, x_{3} ; S_{-\tau}^{l} F_{1}, S_{-\tau}^{2} F_{2}\right)= & {\left[H_{3}^{o}+\psi_{12} ; F_{3}^{l}\left(x_{1}, x_{2}, x_{3} ; S_{-\tau}^{l} F_{1}, S_{-\tau}^{2} F_{2}\right)\right] } \\
& +n \int\left[\Psi_{34} ; S_{-\tau}^{l} F_{1}\left(x_{3}\right) F_{3}^{l}\left(x_{1}, x_{2}, x_{4} ; S_{-\tau}^{1} F_{1}, S_{-\tau}^{2} F_{2}\right)\right] d x_{4} \\
& +\left[\psi_{13}+\psi_{23} ; S_{-\tau}^{2} F_{2}\left(x_{1}, x_{2}\right) S_{-\tau}^{l} F_{1}\left(x_{3}\right)\right] \\
& +n \int\left[\psi_{14}+\Psi_{24} ; S_{-\tau}^{2} F_{2}\left(x_{1}, x_{2}\right) f_{2}^{l}\left(x_{3}, x_{4} ; S_{-\tau}^{1} F_{1}\right)\right] d_{4},
\end{aligned}
$$

where we have replaced $F_{1}$ by $S_{-\tau}^{1} F_{1}$ and $F_{2}$ by $S_{-\tau}^{2} F_{2}$.

Note that in (67) $\tau$ is not the time variable $t$. Here, $t$ occurs as a parameter inside $F_{1}$ and $F_{2}$, and $T$ is a dummy variable introduced to give analytical meaning to $D_{0}$. Equation 67 holds for any value of $\tau$; we shall pick the value of $\tau$ that is most convenient to us. Because of the adiabatic hypothesis and the implications of it, $t$ lost its position as a variable and became a parameter in the equation for $F_{s}$. The $t$-dependence has become a functional dependence on $F_{1}$ and $F_{2}$.

In order to make the functional substitution clear, (67) is more general than is necessary. Since we have assumed that $F_{1}$ has no spacial dependence, we can use

$$
S_{-\tau}^{l} F_{1}\left(\bar{p}_{3}\right)=F_{1}\left(\bar{p}_{3}\right)
$$

throughout this report.

\subsection{SOLUTION OF THE EQUATION FOR $\mathrm{F}_{3}^{1}$}

We shall now solve the equation for $F_{3}^{1}$ for use in the equation for $F_{2}$ without expanding $\mathrm{F}_{2}$ in $\mathrm{g}$; however, we must expand $\mathrm{F}_{2}$ in order to obtain its solution. Some of these operator techniques were developed by Dupree. ${ }^{11}$ 
To proceed, go back to (79) and use (80), to obtain

$$
\begin{aligned}
\frac{\partial}{\partial \tau} \mathrm{F}_{3}^{\mathrm{l}}\left(\mathrm{x}_{1}, \mathrm{x}_{2}, \mathrm{x}_{3} ; \mathrm{F}_{1}, \mathrm{~S}_{-\tau}^{2} \mathrm{~F}_{2}\right)= & {\left[\mathrm{H}_{3}^{\mathrm{o}}+\psi_{12} ; \mathrm{F}_{3}^{1}\left(\mathrm{x}_{1}, \mathrm{x}_{2}, \mathrm{x}_{3} ; \mathrm{F}_{1}, \mathrm{~S}_{-\tau}^{2} \mathrm{~F}_{2}\right)\right] } \\
& +\mathrm{n} \int\left[\Psi_{34} ; \mathrm{F}_{1}\left(\overrightarrow{\mathrm{p}}_{3}\right) \mathrm{F}_{3}^{1}\left(\mathrm{x}_{1}, \mathrm{x}_{2}, \mathrm{x}_{4} ; \mathrm{F}_{1}, \mathrm{~S}_{-\tau}^{2} \mathrm{~F}_{2}\right)\right] \mathrm{dx_{4 }} \\
& +\left[\Psi_{13}+\Psi_{23} ; \mathrm{S}_{-\tau}^{2} \mathrm{~F}_{2}\left(\mathrm{x}_{1}, \mathrm{x}_{2}\right) \mathrm{F}_{1}\left(\overline{\mathrm{p}}_{3}\right)\right] \\
& +\mathrm{n} \int\left[\Psi_{14}+\Psi_{24} ; \mathrm{S}_{-\tau}^{2} \mathrm{~F}_{2}\left(\mathrm{x}_{1}, \mathrm{x}_{2}\right) \mathrm{f}_{2}^{1}\left(\mathrm{x}_{3}, \mathrm{x}_{4} ; \mathrm{F}_{1}\right)\right] \mathrm{dx_{4 }} .
\end{aligned}
$$

For the moment abbreviate

$$
F_{3}^{1}\left(x_{1}, x_{2}, x_{3} ; F_{1}, S_{-\tau}^{2} F_{2}\right) \equiv F_{3}^{l}(\tau),
$$

where the other dependences are understood. Using (22), we may then write (69)

$$
\frac{\partial}{\partial \tau} S_{+\tau}^{2} F_{3}^{1}(\tau)+L S_{+\tau}^{2} F_{3}^{1}(\tau)=S_{+\tau}^{2} \phi(\tau) \text {. }
$$

Here, the operator $L$ acting on any function $\phi\left(x_{3}\right)$ is

$$
L \phi\left(x_{3}\right) \equiv-\left[\mathrm{H}_{1}^{\mathrm{o}} ; \phi\left(\mathrm{x}_{3}\right)\right]-\frac{\partial \mathrm{F}_{1}\left(\overrightarrow{\mathrm{p}}_{3}\right)}{\partial \overrightarrow{\mathrm{p}}_{3}} \cdot \frac{\partial}{\partial \widehat{\mathrm{q}}_{3}} \int \psi_{34} \phi\left(\mathrm{x}_{4}\right) \mathrm{dx_{4 }},
$$

and $\phi(\tau)$ is the source term made up of the last two terms of (69). Note that $S_{T}^{2}$ and $L$ commute since they operate on different coordinates. We call $\mathrm{L}$ the Landau operator since

$$
\frac{\partial f\left(x_{3}, t\right)}{\partial t}+L f\left(x_{3}, t\right)=0
$$

is the equation involving $L$, which has been discussed in detail by Landau. ${ }^{4}$

Writing out the arguments, we obtain the formal solution of (70):

$$
\begin{aligned}
F_{3}^{l}\left(x_{1}, x_{2}, x_{3} ; F_{1}, S_{-\tau}^{2} F_{2}\right)= & e^{-L \tau} s_{-\tau}^{2} \int_{0}^{\tau} d \tau^{\prime} e^{L \tau^{\prime}} S_{\tau^{\prime}}^{2}\left(x_{1}, x_{2}, x_{3} ; F_{1}, S_{-\tau^{\prime}}^{2} F_{2}\right) \\
& +e^{-L \tau} S_{-\tau}^{2} \phi\left(x_{1}, x_{2}, x_{3} ; F_{1}, F_{2}\right),
\end{aligned}
$$

where we use the fact that $L$ does not depend on $T$ when $F_{1}$ has no $\vec{q}$-dependence.

In (72) let $F_{2}-S_{+\tau}^{2} F_{2}$, use the inversion property of the $S_{-\tau}$ operator, and let $\tau^{\prime} \rightarrow \tau-\tau^{\prime}$, to obtain

$$
\begin{aligned}
F_{3}^{1}\left(x_{1}, x_{2}, x_{3} ; F_{1}, F_{2}\right)= & \int_{0}^{\tau} e^{-L \tau^{\prime}} S_{-\tau^{\prime}}^{2} \phi\left(x_{1}, x_{2}, x_{3} ; F_{1}, S_{\tau^{\prime}}^{2} F_{2}\right) d \tau^{\prime} \\
& +e^{-L \tau} S_{-\tau^{2} \phi\left(x_{1}, x_{2}, x_{3} ; F_{1}, F_{2}\right)}
\end{aligned}
$$


The left-hand side of (73) is independent of $\tau$, and thus we are free to pick $\tau$ arbitrarily. We pick $T=\infty$ and thus remove the initial condition term because by (58) $\mathrm{F}_{3}^{1}$ must go to zero for infinite separation of 1 and 2 .

We then have a new understanding of the adiabatic hypothesis and the $\mathrm{D}_{\mathbf{r}}$ expansion. We shall see that (73) implies that, to this order of $g$, the correlations are calculated by integrating along unperturbed (that is, zero-order) orbits while $F_{1}$ and $F_{2}$ are held constant. If $F_{1}$ and $F_{2}$ are known to change in times that are comparable to the time of build-up of correlations, for example in a nonuniform plasma, then this analysis is incorrect. The dummy variable $\tau$ gives us a way of studying this mathematically.

We must interpret the meaning of the operator $e^{-L \tau}$ in $(73)$. For any $\phi\left(x_{3}\right), e^{-L \tau}$ satisfies

$$
\frac{\partial}{\partial \tau} e^{-L \tau} \phi\left(x_{3}\right)+L e^{-L \tau} \phi\left(x_{3}\right) \equiv 0 .
$$

For the moment let

$$
h\left(x_{3}, \tau\right) \equiv e^{-L \tau} \phi\left(x_{3}\right)
$$

for any $\phi\left(x_{3}\right)$, and let us represent by $\overline{\bar{h}}$ the Fourier-Laplace transform

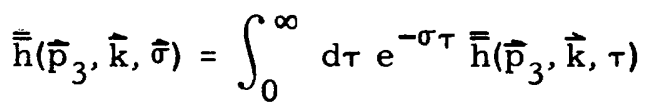

where

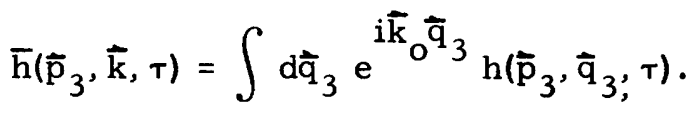

As shown by Landau, ${ }^{4}(75)$ reduces $(74)$ to

$$
\left(\sigma-i \vec{k} \cdot \vec{v}_{3}\right) \overline{\bar{h}}+\frac{m \omega_{p}^{2}}{k^{2}} \vec{k} \cdot \frac{\partial F_{1}\left(p_{3}\right)}{\partial \bar{p}_{3}} \int \overline{\bar{h}} d \vec{p}_{3}^{\prime}=\vec{h}\left(\bar{p}_{3}, \vec{k}, 0\right)
$$

or

$$
\int \overline{\bar{h}} d \vec{p}_{3}=\frac{\int \frac{\overline{\mathrm{h}}\left(\overline{\mathrm{p}}_{3}, \overrightarrow{\mathrm{k}}, 0\right)}{\sigma-\mathrm{i} \overrightarrow{\mathrm{k}} \cdot \overrightarrow{\mathrm{v}}_{3}} d \overrightarrow{\mathrm{p}}_{3}}{1+\mathrm{m} \frac{\omega_{\mathrm{p}}^{2}}{\mathrm{k}^{2}} \int \frac{\overrightarrow{\mathrm{k}} \cdot \frac{\partial \mathrm{F}_{1}}{\partial \overrightarrow{\mathrm{p}}_{3}^{\prime}}}{\sigma-i \overrightarrow{\mathrm{k}} \cdot \overrightarrow{\mathrm{v}}_{3}^{\prime}} \mathrm{d} \overrightarrow{\mathrm{p}}_{3}^{\prime}} .
$$

(We are actually interested only in the integral of $\overline{\bar{h}}$ over $\mathrm{d}_{3}$.) Since Re $\sigma>0$ in the definition of $\overline{\bar{h}}$ in (75), the integral in (77) is defined. To stay above the point $(-L \sigma)$, we take the same integral, with the $\overrightarrow{\mathrm{p}}_{3}^{\prime}$-integration deformed, to be the analytic continuation of the function Re $\sigma \leqslant 0$. All of this computation is identical to Landau's work. 
Finally, inverting the transform, we obtain

$$
\int h d \vec{p}_{3}=\frac{1}{(2 \pi)^{4} i} \int_{-\infty i+\beta}^{\infty i+\beta} d \sigma e^{\sigma \tau} \int d \vec{k} e^{-i \vec{k} \cdot \vec{q}_{3}} \cdot \frac{\int \frac{\bar{h}\left(\vec{p}_{3}, \vec{k}, 0\right)}{\sigma-i \vec{k} \cdot \vec{v}_{3}} d \vec{p}_{3}}{1+L+(\sigma)}
$$

where we define

$$
L_{ \pm}(\sigma) \equiv m \frac{i \omega_{p}^{2}}{k^{2}} \int_{ \pm} \frac{\vec{k} \cdot \frac{\partial F_{1}\left(p_{3}^{\prime}\right)}{\partial-i \vec{k} \cdot \vec{v}_{3}^{\prime}}}{\sigma \vec{p}_{3}}
$$

The plus and minus signs indicate that the contour is to pass above or below the singularity.

Resorting to the definition of $h$, we have

$$
\int e^{-L \tau} \phi\left(x_{3}\right) d \vec{p}_{3}=\frac{1}{(2 \pi)^{4} i} \int_{-i \infty+\beta}^{i \infty+\beta} d \sigma e^{\sigma \tau} \int d \vec{k} e^{-i \vec{k} \cdot \vec{q}_{3}} \frac{\int \frac{\phi\left(\vec{k}, \vec{p}_{3}\right)}{\sigma-i \vec{k} \cdot \vec{v}_{3}^{\prime}} d \vec{p}_{3}}{1+L_{+}(\sigma)}
$$

This implies that

$$
\begin{aligned}
& \int \mathrm{d} \hat{\mathrm{p}}_{3} \mathrm{~F}_{3}^{1}\left(\mathrm{x}_{1}, \mathrm{x}_{2}, \mathrm{x}_{3} ; \mathrm{F}_{1}, \mathrm{~F}_{2}\right) \\
& =\int \mathrm{d} \vec{p}_{3} \int_{0}^{\infty} \mathrm{d} \tau \mathrm{e}^{-\mathrm{L} \tau} \phi\left(\mathrm{s}_{-\tau}^{2} \mathrm{x}_{1}, \mathrm{x}_{2}, \mathrm{x}_{3} ; \mathrm{F}_{1}, \mathrm{~F}_{2}\right) \\
& =\frac{1}{(2 \pi)^{4} i} \int_{0}^{\infty} d \tau \int_{-\infty i+\beta}^{\infty i+\beta} d \sigma e^{\sigma \tau} \int d \vec{k} e^{-i \vec{k} \cdot \bar{q}_{3}} \frac{\int \frac{\bar{\phi}\left(s_{-\tau}^{2} x_{1}, x_{2}, \vec{k}, \vec{p}_{3} ; F_{1}, F_{2}\right)}{\sigma-i \vec{k} \cdot \vec{v}_{3}} d \vec{p}_{3}}{1+L_{+}(\sigma)}
\end{aligned}
$$

where

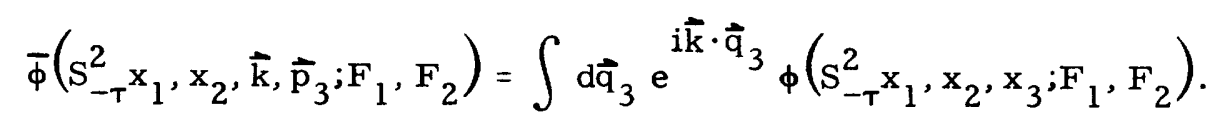

In (80) we have used the fact that

$$
S_{-\tau}^{2}\left(x_{1}, x_{2}, x_{3} ; F_{1}, S_{\tau}^{2} F_{2}\right)=\phi\left(S_{-\tau}^{2} x_{1}, x_{2}, x_{3} ; F_{1}, F_{2}\right)
$$

This follows, since $S_{-\tau}^{2}$ is defined to operate inside the function wherever $x_{1}$ and $x_{2}$ occur. In (81) we interpret the $S_{-\tau}^{2}$ as operating on those $x_{1}$ and $x_{2}$ lying outside the function $F_{2}\left(x_{1}, x_{2}\right)$. 
To continue we must insert the form of $\bar{\phi}$. Remember that we defined

$$
\begin{aligned}
\phi \equiv & {\left[\psi_{23}+\psi_{13} ; \mathrm{F}_{2}\left(\mathrm{x}_{1}, \mathrm{x}_{2}\right) \mathrm{F}_{1}\left(\overline{\mathrm{p}}_{3}\right)\right] } \\
& +\mathrm{n} \int\left[\Psi_{14}+\psi_{24} ; \mathrm{F}_{2}\left(\mathrm{x}_{1}, \mathrm{x}_{2}\right) \mathrm{f}_{2}^{\mathrm{l}}\left(\overline{\mathrm{p}}_{3}, \overline{\mathrm{p}}_{4}, \overline{\left.\mathrm{q}_{2}-\mathrm{q}_{4}\right)}\right] \mathrm{dx_{4 }} .\right.
\end{aligned}
$$

Using these definitions we obtain

$$
\begin{aligned}
& \bar{\phi}\left(\mathrm{x}_{1}, \mathrm{x}_{2}, \overrightarrow{\mathrm{k}}, \overrightarrow{\mathrm{p}}_{3} ; \mathrm{F}_{1}, \mathrm{~F}_{2}\right) \\
& \quad=\frac{4 \pi i \mathrm{e}^{2}}{\mathrm{k}^{2}} \overrightarrow{\mathrm{k}} \cdot\left\{\left(\overrightarrow{\mathrm{d}}_{13} \mathrm{~F}_{2}\left(\mathrm{x}_{1}, \mathrm{x}_{2}\right) \mathrm{F}_{3}\left(\overrightarrow{\mathrm{p}}_{3}\right)+\mathrm{n} \frac{\partial \mathrm{F}_{2}\left(\mathrm{x}_{1}, \mathrm{x}_{2}\right)}{\partial \overrightarrow{\mathrm{p}}_{1}} \int \overline{\mathrm{f}}_{2}\left(\overrightarrow{\mathrm{p}}_{3}, \overrightarrow{\mathrm{p}}_{4}, \overline{\mathrm{k}}\right) \mathrm{d} \overline{\mathrm{p}}_{4}\right) \mathrm{e}^{\mathrm{i \vec { \textrm {k } }} \cdot \overline{\mathrm{q}}_{1}}+(1 \leftrightarrow 2)\right\} .
\end{aligned}
$$

In (83) the notation $(1 \rightarrow-2)$ means that the previous term is repeated with 1 and 2 interchanged,

and

$$
\overline{\mathrm{d}}_{\mathrm{ij}} \equiv \frac{\partial}{\partial \overline{\mathrm{p}}_{i}}-\frac{\partial}{\partial \overline{\mathrm{p}}_{j}}
$$

$$
\overline{\mathrm{f}}_{2}^{\mathrm{l}}\left(\overrightarrow{\mathrm{p}}_{3}, \overline{\mathrm{p}}_{4}, \overrightarrow{\mathrm{k}}\right) \equiv \int \mathrm{d} \overrightarrow{\mathrm{q}} \mathrm{e}^{\mathrm{i} \hat{\mathrm{k}} \cdot \overrightarrow{\mathrm{q}}_{\mathrm{f}}} \mathrm{f}_{2}\left(\overline{\mathrm{p}}_{3}, \overline{\mathrm{p}}_{4}, \overrightarrow{\mathrm{q}}\right)
$$

If $\vec{q}_{1}(-\tau)$ is the position of particle 1 at $-\tau$, given that it was at $\vec{q}_{1}$ at $\tau=0$, with the mechanism of the motion governed by the two-body collision, and similarly for $\vec{q}_{2}(-\tau)$, then (83) becomes

$$
\begin{aligned}
& \bar{\phi}\left(\mathrm{s}_{-\tau}^{2} \mathrm{x}_{1}, \mathrm{x}_{2}, \overrightarrow{\mathrm{k}}, \overrightarrow{\mathrm{p}}_{3} ; \mathrm{F}_{1}, \mathrm{~F}_{2}\right) \\
& \quad=\frac{4 \pi i \mathrm{e}^{2}}{\mathrm{k}^{2}} \mathrm{k} \cdot\left\{\left(\overrightarrow{\mathrm{d}}_{13} \mathrm{~F}_{2}\left(\mathrm{x}_{1}, \mathrm{x}_{2}\right) \mathrm{F}_{3}\left(\overrightarrow{\mathrm{p}}_{3}\right)+\mathrm{n} \frac{\partial \mathrm{F}_{2}\left(\mathrm{x}_{1}, \mathrm{x}_{2}\right)}{\partial \overrightarrow{\mathrm{p}}_{1}} \int \overline{\mathrm{f}}_{2}\left(\overline{\mathrm{p}}_{3}, \overline{\mathrm{p}}_{4}, \overline{\mathrm{k}}\right) \mathrm{d} \overrightarrow{\mathrm{p}}_{4}\right) \mathrm{e}^{\mathrm{i} \overrightarrow{\mathrm{k}} \cdot \overrightarrow{\mathrm{q}}_{1}(-\tau)}+(1 \rightarrow 2)\right\} .
\end{aligned}
$$

Let us now discuss the interpretation of some of the integrals appearing in (80). Note that the $\tau$-dependence in $\bar{\phi}$ is in the form of an exponential with an imaginary argument. Thus the $\tau$-integration can proceed only if $\beta$ is negative. Since the inversion in the $\sigma$-plane must go to the right of all singularities, $\beta$ can be negative only if all of the zeros of the denominator lie to the left of the imaginary axis. For a wide range of $F_{1}\left(\bar{p}_{3}\right)$ this is true as long as $|k|>0.13$ Thus we exclude an infinitesimal region from the origin of $\vec{k}$. Under these restrictions we may proceed with the $\tau$-integration, followed by the $\sigma$, then by the $\overrightarrow{\mathbf{k}}$.

Those distribution functions that give zeros of $1+L_{+}(\sigma)$ in the right-half plane present a difficult problem. For these situations the past is not damped out, and the current value of $\mathrm{F}_{3}^{\mathrm{l}}$ must depend on the initial conditions - a situation that is incompatible 
with the adiabatic hypothesis. ${ }^{11}$ It would appear that the hypothesis is invalid for plasmas containing these instabilities. In order to proceed we shall limit ourselves to the more well behaved functions $F_{1}$. As Baccus shows, ${ }^{13}$ these are all single-humped momentum distributions.

A physical understanding of why some distributions $F_{1}(\bar{p})$ give rise to damped solutions and others do not can be seen from the arguments used to explain Landau damping and plasma instabilities. ${ }^{14}$ Because the shielding integral (the L operator) in (70) is the same as that considered by Landau ${ }^{4}$ in his use of the Vlasov equation, we obtained Landau's characteristic denominator $1+L_{+}(\sigma)$ and can use his analysis of the study of waves to study the growth of correlations.

For the $\vec{k}^{\text {th }}$ partial wave in the analysis of wave motion in a plasma, those particles traveling at the phase velocity of the wave will see a constant field. If

$$
\left.\overrightarrow{\mathrm{k}} \cdot \frac{\partial}{\partial \overrightarrow{\mathrm{p}}} \mathrm{F}_{1}(\overrightarrow{\mathrm{p}})\right|_{\mathrm{v}=\sigma / \mathrm{ik}}
$$

is negative, more particles will be speeded up than are slowed down, and damping of the wave will result. If this derivative is positive, energy will be fed from the particles to the wave, and a growing wave will result.

In the problem considered, the plasma is homogeneous and the $\vec{k}$-analysis refers to the coordinates $\bar{q}_{1}-\bar{q}_{3}$ and $\bar{q}_{2}-\bar{q}_{3}$. Whereas for waves we had damped or increasing energy, in the problem considered we have decreasing or increasing correlation.

Using the form of $\phi$ in (69) and the meaning of the $S_{-\tau}^{2}$ operators, we have

$$
\begin{aligned}
\phi\left(s_{-\tau}^{2} x_{1}, x_{2}, x_{3}, F_{1}, F_{2}\right)= & {\left[S_{-\tau}^{2}\left(\psi_{13}+\psi_{23}\right) ; F_{2}\left(x_{1}, x_{2}\right) F_{1}\left(x_{3}\right)\right] } \\
& +n \int\left[s_{-\tau}^{2}\left(\psi_{14}+\psi_{24}\right) ; F_{2}\left(x_{1}, x_{2}\right) f_{2}^{1}\left(x_{3}, x_{4}\right)\right] d x_{4} .
\end{aligned}
$$

Throughout this analysis we have assumed that we never have a three-body collision, that is, that the interactions of all pairs except 1 and 2 are of order $g$. This assumption implies that the distances $\left|\overrightarrow{\mathrm{q}}_{1}-\overrightarrow{\mathrm{q}}_{3}\right|,\left|\overrightarrow{\mathrm{q}}_{2}-\mathrm{q}_{3}\right|,\left|\overrightarrow{\mathrm{q}}_{1}-\overrightarrow{\mathrm{q}}_{4}\right|$, and $\left|\overrightarrow{\mathrm{q}}_{2}-\mathrm{q}_{4}\right|$ are large in the sense of the $g$ expansion. The $S_{-\tau}$ operators in $(85)$ have the effect of

$$
\mathrm{S}_{-\tau}^{2} \psi_{13}\left({\overrightarrow{\mathrm{q}_{1}-\mathrm{q}_{3}}}\right)=\psi_{13}\left(\overrightarrow{\mathrm{q}_{1}(-\tau)-\mathrm{q}_{3}}\right) \text {. }
$$

The change in $\psi_{13}$ will become important only when $\left|{\overline{q_{1}}(-\tau)-q_{1}}_{1}\right|$ becomes of the order of $\left|\bar{q}_{1}-\mathrm{q}_{3}\right|$. By assumption this change takes a long time, a time that is sufficient for 1 and 2 to be far apart. For example, we assume that $\left|\bar{q}_{2}-q_{3}\right|$ and $\left|\bar{q}_{2}-q_{3}\right| \geqslant \sqrt{e^{2} \lambda_{D} / k T}$. At this separation the potential $\psi_{12}$ has the magnitude

$$
\psi_{12}\left(\sqrt{\frac{e^{2} \lambda_{D}}{k T}}\right) \cong k T \sqrt{\frac{e^{2}}{k T \lambda_{D}}},
$$


which is much smaller than the average kinetic energy $\mathrm{kT}$. Thus for those values of $T$ in (86) which have any effect, 1 and 2 can be considered to be interacting only weakly. In (86) we will specifically use the fact that as $q_{1}$ is projected backward in time, the difference $\left|\vec{q}_{1}-\vec{q}_{3}\right|$ is relatively insensitive to the change in $\vec{q}_{1}$ while $\vec{q}_{1}$ is in the immediate vicinity of $\overline{\mathrm{q}}_{2}$. A similar argument holds for $\psi_{23}$ by interchanging 1 and 2 .

These statements can be made quantitative by introducing in (86) the operator $S_{-\tau}^{2}$ that projects 1 and 2 backward with zero interaction, that is, along straight lines and with constant velocity,

$$
\mathrm{S}_{-\tau}^{2} \psi_{13}\left(\overline{\mathrm{q}_{1}-\mathrm{q}_{3}}\right) \equiv \mathrm{S}_{-\tau}^{2} \dot{\mathrm{S}}_{\tau}^{2} \dot{\mathrm{S}}_{-\tau}^{2} \psi_{13}\left(\overline{\mathrm{q}_{1}-\overline{\mathrm{q}}_{3}}\right)=\mathrm{S}_{-\tau}^{2} \dot{\mathrm{S}}_{\tau}^{2} \psi_{13}\left(\overline{\mathrm{q}_{1}-\mathrm{v}_{1} \tau-\mathrm{q}_{3}}\right)
$$

As we shall see below, for large values of $\tau$, the product $S_{-\tau}^{2} \dot{S}_{\tau}^{2}$ becomes stationary, independent of $\tau$. This fact, coupled with the preceding discussion, will be used to approximate $(87)$.

To understand the foregoing assertions, consider the operation on the velocity of 1 or 2

$$
\lim _{\tau \rightarrow \infty} S_{-\tau}^{2} \vec{v}_{i}
$$

This will approach a constant limit defined as

$$
\lim _{\tau \rightarrow \infty} S_{-\tau}^{2} \vec{v}_{i} \equiv \vec{V}_{i}\left(x_{1}, x_{2}\right) \text {. }
$$

By its definition $\vec{V}_{i}\left(\vec{P}_{i}=m \vec{V}_{i}\right)$ is the velocity (momentum) that the $i^{\text {th }}$ particle had in the distant past before undergoing the two-body collision, given that the colliding particles have current coordinates $x_{1}$ and $x_{2}$. For this reason we shall refer to it as the precollision velocity (momentum).

Equation 88 proceeds at such a rate that

$$
\lim _{\tau \rightarrow \infty} \tau\left(\overrightarrow{\mathrm{V}}_{\mathrm{i}}-\mathrm{S}_{-\tau}^{2} \overrightarrow{\mathrm{v}}_{\mathrm{i}}\right) \rightarrow 0
$$

This condition ensures the existence of the limit

$$
\lim _{\tau \rightarrow \infty}\left(s_{-\tau}^{2} \vec{q}_{i}+\vec{V}_{i} \tau\right)=\lim _{\tau \rightarrow \infty}\left(\vec{q}_{i}+\int_{0}^{\tau}\left(\vec{v}_{i}-s_{-\tau^{\prime}}^{2} \vec{v}_{i}\right) d \tau^{\prime}\right) \rightarrow \vec{Q}_{i}
$$

where

$$
\vec{Q}_{i}\left(x_{1}, x_{2}\right) \equiv \vec{q}_{i}+\int_{0}^{\infty}\left(\vec{v}_{i}-S_{-\tau}^{2} \vec{v}_{i}\right) d \tau
$$

To find the position $Q_{i}\left(x_{1}, x_{2}\right)$, start at the point $\bar{q}_{i}$, project backward $\tau$ seconds along the trajectories of a two-body interaction, and project forward $\tau$ seconds with the constant precollision velocity $\vec{V}_{i}$. In the limit $\tau \rightarrow \infty$, this procedure will give the position that the $i^{\text {th }}$ particle would have had with no collision interaction. For this reason we call $\vec{Q}_{i}$ the undeflected position. 
Now consider for any $\phi\left(x_{1}, x_{2}\right)$

$$
\begin{aligned}
& \lim _{\tau \rightarrow \infty} \mathrm{S}_{-\tau}^{2} \dot{\mathrm{S}}_{\tau}^{2} \phi\left(\overrightarrow{\mathrm{p}}_{1}, \overrightarrow{\mathrm{p}}_{2}, \overrightarrow{\mathrm{q}}_{1}, \overrightarrow{\mathrm{q}}_{2}\right) \\
& =\lim _{\tau \rightarrow \infty} s_{-\tau}^{2} \phi\left(\vec{p}_{1}, \vec{p}_{2},{\overrightarrow{q_{1}-v_{1}}}_{1},{\overrightarrow{q_{2}}}^{-v_{2}} \tau\right) \\
& =\lim _{\tau \rightarrow \infty} \phi\left(S_{-\tau^{2}}^{2} \vec{p}_{1}, S_{-\tau^{2}}^{2} \vec{p}_{2}, q_{1}+\int_{0}^{\tau}\left(S_{-\tau^{2} v_{1}}^{2}-S_{-\tau^{\prime}}^{2} v_{1}\right) d \tau^{\prime}, q_{2}+\int_{0}^{\tau}\left(S_{-\tau^{2}}^{2} \vec{v}_{2}-S_{-\tau^{\prime}}^{2}, v_{2}\right) d \tau^{\prime}\right) \\
& =\phi\left(\overrightarrow{\mathrm{P}}_{1}, \overrightarrow{\mathrm{P}}_{2}, \overrightarrow{\mathrm{Q}}_{1}, \overrightarrow{\mathrm{Q}}_{2}\right) \\
& =\phi\left(X_{1}, X_{2}\right) \text {. }
\end{aligned}
$$

We define the 6-dimensional vector $X_{i}=\left\{P_{i}, Q_{i}\right\}$.

Notice that the transformation from $\left(x_{1}, x_{2}\right)$ to $\left(X_{1}, X_{2}\right)$ is a contact transformation, since it is obtained from a product of transformations governed by Hamiltonians. This property will be useful because of the invariance of the Poisson brackets with respect to a contact transformation.

To approximate the product $\mathrm{S}_{-\tau}^{2} \dot{\mathrm{S}}_{\tau}^{2}$ on any function $\phi\left(\mathrm{x}_{1}, \mathrm{x}_{2}\right)$, we may expand the result around $\tau=\infty$ :

$$
\begin{aligned}
& S_{-\tau}^{2} \dot{\mathrm{S}}_{\tau}^{2} \phi\left(\mathrm{x}_{1}, \mathrm{x}_{2}\right)=\left\{1+\left(\mathrm{S}_{-\tau}^{2} \overrightarrow{\mathrm{P}}_{1}-\overrightarrow{\mathrm{P}}_{1}\right) \cdot \frac{\partial}{\partial \overrightarrow{\mathrm{P}}_{1}}+\left(\mathrm{S}_{-\tau}^{2} \overrightarrow{\mathrm{P}}_{2}-\overrightarrow{\mathrm{P}}_{2}\right) \cdot \frac{\partial}{\partial \overrightarrow{\mathrm{P}}_{2}}+\left(\mathrm{S}_{-\tau}^{2} \dot{\mathrm{S}}_{\tau}^{2} \overrightarrow{\mathrm{q}}_{1}-\mathrm{Q}_{1}\right) \cdot \frac{\partial}{\partial \overrightarrow{\mathrm{Q}}_{1}}\right. \\
& \left.+\left(\mathrm{S}_{-\tau}^{2} \dot{\mathrm{S}}_{\tau}^{2} \overrightarrow{\mathrm{q}}_{2}-\overrightarrow{\mathrm{Q}}_{2}\right) \cdot \frac{\partial}{\partial \overrightarrow{\mathrm{Q}}_{2}}\right\} \mathrm{S}_{-\infty}^{2} \stackrel{\mathrm{S}}{\mathrm{S}}_{-\infty}^{2} \phi\left(\mathrm{x}_{1}, \mathrm{x}_{2}\right)+\text { higher -order terms. }
\end{aligned}
$$

Application of this to $\psi_{13}\left(\overline{q_{1}-v_{1} \tau-q_{3}}\right)$ yields

$$
\begin{aligned}
\mathrm{S}_{-\tau}^{2} \dot{\mathrm{S}}_{\tau}^{2} \psi_{13}\left(\overline{\mathrm{q}_{1}-\mathrm{v}_{1} \tau-\mathrm{q}_{3}}\right) \cong & \psi_{13}\left(\overline{\mathrm{Q}_{1}-\mathrm{V}_{1} \tau-\mathrm{q}_{3}}\right) \\
& +\left(\mathrm{S}_{-\tau} \overline{\mathrm{q}}_{1}-\left(\overline{\mathrm{Q}_{1}-\bar{V}_{1}} \tau\right)\right) \cdot \frac{\partial}{\partial \mathrm{Q}_{1}} \psi_{13}\left(\overline{\mathrm{Q}_{1}-\mathrm{V}_{1} \tau-\mathrm{q}_{3}}\right)+\ldots .
\end{aligned}
$$

The coefficient of the second term is, as a function of $\tau$, the deflection of particle 1 along the path of its collision with 2. By using the specific form of $\psi$, the ratio of the second to the first term is of order

$$
\frac{\left|s_{-\tau}^{2} \overline{\mathrm{q}}_{1}-\left(\overrightarrow{\mathrm{Q}_{1}-\vec{V}_{1}} \tau\right)\right|}{\left|\overline{\mathrm{Q}}_{1}-\overrightarrow{\mathrm{V}}_{1} \tau-\overline{\mathrm{q}}_{3}\right|} \leqslant \frac{\left|\overrightarrow{\mathrm{q}_{1}-\mathrm{Q}_{1}}\right|}{\left|\overrightarrow{\mathrm{Q}_{1}-\mathrm{q}_{3}}\right|} .
$$

The quantity $\left|\overrightarrow{Q_{1}-q_{3}}\right|$ is large for the same reason that $\left|\overrightarrow{q_{1}-q_{3}}\right|$ is large, and it is of the same order of magnitude. The ratio is then small for all situations except those in which 1 and 2 are widely separated but have had a collision in the past (that is, $\left|\overline{q_{1}-Q_{1}}\right|$ 
is large enough to be in the range $\left.\left|\overrightarrow{Q_{1}-q_{3}}\right|\right)$. For 1 and 2 close together and 1 and 2 experiencing a close collision, the ratio (89) is small.

We shall ignore the exception noted above and assume that the ratio is small for all situations of interest. We shall see that those situations for which (89) is not small have negligible contribution to the problems of our interest.

These approximations are in the source term of $\mathrm{F}_{3}^{\mathrm{l}}$. If we were to consider corrections, they would occur as new (small) source terms and be additive.

We shall then use the fact that the approximation

$$
\mathrm{S}_{-\tau}^{2} \psi_{13}\left(\overline{\mathrm{q}}_{1}-\mathrm{q}_{3}\right) \cong \psi_{13}\left(\overline{\mathrm{Q}}_{1}-\overline{\mathrm{V}}_{1} \tau-\overline{\mathrm{q}}_{3}\right)
$$

is a valid outcome of the $g$ expansion; and similarly for $S_{-T}^{2} \psi_{23}$. Since in the integration over $\mathrm{dx}_{4}$ in the source term $\phi$ we assumed that $\left|\overrightarrow{\mathrm{q}}_{1}-\mathrm{q}_{4}\right|$ and $\left|\overrightarrow{\mathrm{q}}_{2}-\mathrm{q}_{4}\right|$ are large (that is, we cut off the integral), we can make similar expansions for $S_{-\tau}^{2} \psi_{14}$ and $S_{-\tau}^{2} \psi_{24}$.

This step represents the real departure from the analysis when all particle interactions are assumed small. Rather than the approximation (90), these treatments have implicitly used the approximation

$$
\mathrm{S}_{-\tau}^{2} \dot{\mathrm{S}}_{-\tau}^{2} \cong 1
$$

or

$$
\mathrm{S}_{-\tau}^{2} \psi_{13}\left(\overline{\mathrm{q}_{1}-\mathrm{q}_{3}}\right) \stackrel{\sim}{=} \psi_{13}\left(\overline{\mathrm{q}_{1}-\mathrm{v}_{1}-\mathrm{q}_{3}}\right)
$$

By the discussion above we see that these approximations are valid only when $\psi_{12}$ is weak at present and in the past. Use of (91) and the following procedure would lead directly to the large-separation solution.

We may then use these approximations in (84). Since we effectively make the transformation $\bar{q}_{i}-\bar{Q}_{i}$ inside $\psi$, to retain the form of the Poisson brackets we must transform $\partial / \partial \widehat{\mathrm{p}}_{\mathrm{i}} \rightarrow \partial / \partial \widehat{\mathrm{P}}_{\mathrm{i}}$. With these changes, (84) becomes

$$
\begin{aligned}
& \bar{\phi}\left(\mathrm{s}_{-\tau}^{2} \mathrm{x}_{1}, \mathrm{x}_{2}, \overrightarrow{\mathrm{k}}, \overrightarrow{\mathrm{p}}_{3} ; \mathrm{F}_{1}, \mathrm{~F}_{2}\right) \cong \frac{4 \pi i \mathrm{e}^{2}}{\mathrm{k}^{2}} \overrightarrow{\mathrm{k}} \cdot\left\{\left(\overrightarrow{\mathrm{D}}_{13} \mathrm{~F}_{2}\left(\mathrm{x}_{1}, \mathrm{x}_{2}\right) \mathrm{F}_{1}\left(\overrightarrow{\mathrm{p}}_{3}\right)\right.\right. \\
& \left.\left.+n \frac{\partial F_{2}\left(x_{1}, x_{2}\right)}{\partial \overrightarrow{\mathrm{P}}_{1}} \int-f_{2}^{1}\left(\vec{p}_{3}, \overrightarrow{\mathrm{p}}_{4}, \vec{k}\right) d \overrightarrow{\mathrm{p}}_{4}\right) e^{i \vec{k} \cdot\left(\overrightarrow{\mathrm{Q}}^{-\vec{V}_{1}} \tau\right)}+(1-2)\right\}
\end{aligned}
$$

where $\overrightarrow{\mathrm{D}}_{13} \equiv \frac{\partial}{\partial \overrightarrow{\mathrm{P}}_{1}}-\frac{\partial}{\partial \overrightarrow{\mathrm{p}}_{3}}$ and $\overrightarrow{\mathrm{D}}_{23} \equiv \frac{\partial}{\partial \overrightarrow{\mathrm{P}}_{2}}-\frac{\partial}{\partial \overrightarrow{\mathrm{p}}_{3}}$.

Using (92) in (80), we obtain another expression for $F_{3}^{1}$,

$\int F_{3}^{1}\left(x_{1}, x_{2}, x_{3} ; F_{1}, F_{2}\right) d \vec{p}_{3}=\frac{4 \pi e^{2}}{(2 \pi)^{4}} \int_{0}^{\infty} d \tau \int_{-i \infty+\beta}^{i \infty+\beta} d \sigma e^{\sigma \tau} \int \frac{d \vec{k} e^{-i \vec{k} \cdot \vec{q}_{3}}}{k^{2}} \frac{1}{1+L_{+}(\sigma)} \int \frac{d \vec{p}_{3}}{\sigma-i \vec{k} \cdot \hat{v}_{3}}$ 
$\vec{k} \cdot\left\{\left(\vec{D}_{13} F_{2}\left(x_{1}, x_{2}\right) F_{1}\left(\vec{p}_{3}\right)+n \frac{\partial F_{2}\left(x_{1}, x_{2}\right)}{\partial \vec{P}_{1}} \int \bar{f}_{2}^{1}\left(\vec{p}_{3}, \vec{p}_{4}, \vec{k}\right) d \vec{p}_{4}\right) e^{i \vec{k} \cdot\left(\vec{Q}_{1}^{-V_{1}} \tau\right)}+(1 \leftrightarrow 2)\right\}$

If $\beta<0$, we may carry out the $\tau$-integration, which will bring down $\left(\sigma-i \vec{k} \cdot \overline{\mathrm{V}}_{1}\right)^{-1}$ and $\left(\sigma-\mathrm{i} \overrightarrow{\mathrm{k}} \cdot \overrightarrow{\mathrm{V}}_{2}\right)^{-1}$ in the two terms. Note that the approximation (90) was made to make the $\tau$-integration possible. For those functions $F_{1}\left(\vec{p}_{3}\right)$ for which $1+L_{+}(\sigma)$ has no poles in the right-half plane, the $\sigma$-integration can be closed in the right-half plane and will only enclose poles at $i \vec{k} \cdot \vec{V}_{1}$ and $i \vec{k} \cdot \vec{v}_{2}$. Carrying out these two steps, we obtain

$$
\begin{aligned}
& \int F_{3}^{1}\left(x_{1}, x_{2}, x_{3} ; F_{1}, F_{2}\right) d \vec{p}_{3} \\
& =\frac{4 \pi \mathrm{e}^{2} \mathrm{i}}{(2 \pi)^{3}} \int \frac{\mathrm{d} \overrightarrow{\mathrm{k}} \mathrm{e}^{-\mathrm{i} \overrightarrow{\mathrm{k}} \cdot \overrightarrow{\mathrm{q}}_{3}}}{\mathrm{k}^{2}} \overrightarrow{\mathrm{k}} \cdot\left\{\int_{+} \frac{\mathrm{d} \overrightarrow{\mathrm{p}}_{3}}{\overrightarrow{\mathrm{k}} \cdot\left(\overline{\mathrm{V}}_{1} \mathrm{v}_{3}\right.}\right)\left(\overrightarrow{\mathrm{D}}_{13} \mathrm{~F}_{2}\left(\mathrm{x}_{1}, \mathrm{x}_{2}\right) \mathrm{F}_{1}\left(\overrightarrow{\mathrm{p}}_{3}\right)\right. \\
& \left.\left.+n \frac{\partial \mathrm{F}_{2}\left(\mathrm{x}_{1}, \mathrm{x}_{2}\right)}{\partial \overrightarrow{\mathrm{P}}_{1}} \int \mathrm{f}_{2}^{1}\left(\overrightarrow{\mathrm{p}}_{3}, \overrightarrow{\mathrm{p}}_{4}, \overrightarrow{\mathrm{k}}\right) d \overrightarrow{\mathrm{p}}_{4}\right) \frac{\mathrm{e}^{\mathrm{i} \overrightarrow{\mathrm{k}} \cdot \overrightarrow{\mathrm{Q}}_{1}}}{1+\mathrm{L}_{+}\left(\mathrm{i} \overrightarrow{\mathrm{k}} \cdot \overrightarrow{\mathrm{V}}_{1}\right)}+(1 \leftrightarrow 2)\right\} .
\end{aligned}
$$

The $\int_{+}$means that the $\overrightarrow{\mathrm{p}}_{3}$-integration is to stay above the poles, since $(-\mathrm{i} \sigma)$ was to be below the axis.

We wish to use this result in an integral of the form

$$
\mathrm{n} \int\left[\psi_{13}\left(\overline{\mathrm{q}}_{1}-\mathrm{q}_{3}\right)+\psi_{23}\left(\overline{\mathrm{q}}_{2}-\mathrm{q}_{3}\right) ; \mathrm{F}_{3}^{1}\left(\mathrm{x}_{1}, \mathrm{x}_{2}, \mathrm{x}_{3} ; \mathrm{F}_{1}, \mathrm{~F}_{2}\right)\right] \mathrm{d} \mathrm{x}_{3} \text {, }
$$

where we are faced with the integration of a coordinate that is assumed to be far removed from $\overrightarrow{\mathrm{q}}_{1}$ and $\mathrm{q}_{2}$. Accordingly, we may approximate under the integral

$$
\mathrm{n} \int\left[\psi_{13}\left({\overrightarrow{Q_{1}-q_{3}}}\right)+\psi_{23}\left({\overrightarrow{Q_{2}-q_{3}}}\right) ; F_{3}^{1}\left(x_{1}, x_{2}, x_{3} ; F_{1}, F_{2}\right)\right] d x_{3} \text {. }
$$

This approximation can be analyzed exactly as before, that is,

$$
\psi_{13}\left(\overrightarrow{\mathrm{Q}}_{1}-\mathrm{q}_{3}\right) \cong \psi_{13}\left(\overrightarrow{\mathrm{q}}_{1}-\mathrm{q}_{3}\right)+\left(\overrightarrow{\mathrm{Q}_{1}-\mathrm{q}_{1}}\right) \cdot \frac{\partial}{\partial \overrightarrow{\mathrm{q}}_{1}} \psi_{13}\left({\overrightarrow{\mathrm{q}_{1}-\mathrm{q}_{3}}}\right)+\ldots
$$

The error term is of order

$$
\frac{\left|\overrightarrow{Q_{1}-q_{1}}\right|}{\left|{\overrightarrow{q_{1}}-\mathrm{q}_{3}}\right|}
$$

which has the same characteristics as (89).

Putting (94) into (95) yields the interaction integral in the equation for $\mathrm{F}_{2}$ : 


$$
\begin{aligned}
& \frac{\partial \mathrm{F}_{2}\left(\mathrm{x}_{1}, \mathrm{x}_{2}\right)}{\partial \mathrm{t}}=\left[\mathrm{H}_{2}^{\mathrm{O}}+\Psi_{12} ; \mathrm{F}_{2}\left(\mathrm{x}_{1}, \mathrm{x}_{2}\right)\right]-\mathrm{g} \frac{\mathrm{n}\left(4 \pi \mathrm{e}^{2}\right)^{2} \mathrm{i}}{(2 \pi)^{3}} \int \frac{\mathrm{d} \overrightarrow{\mathrm{k}}}{\mathrm{k}^{2}} \overline{\mathrm{k}} \text {. } \\
& \left\{\frac { \partial } { \partial \overline { \mathrm { P } } _ { 1 } } \frac { 1 } { 1 + \mathrm { L } _ { + } ( \mathrm { i } \overline { \mathrm { k } } \cdot \overline { \mathrm { V } } _ { 1 } ) } \int _ { + } \frac { \mathrm { d } \overline { \mathrm { p } } _ { 3 } } { \overline { \mathrm { k } } \cdot ( \overline { \mathrm { V } } _ { 1 } - \vec { \mathrm { v } } _ { 3 } ) } \mathrm { k } \cdot \left(\overrightarrow{\mathrm{D}}_{13} \mathrm{~F}_{2}\left(\mathrm{x}_{1}, \mathrm{x}_{2}\right) \mathrm{F}_{1}\left(\overline{\mathrm{p}}_{3}\right)\right.\right. \\
& \left.+n \frac{\partial F_{2}\left(x_{1}, x_{2}\right)}{\partial \overrightarrow{\mathrm{P}}_{1}} \int \bar{f}_{2}^{1}\left(\vec{p}_{3}, \vec{p}_{4}, \vec{k}\right) d \vec{p}_{4}\right) \\
& +e^{i \vec{k} \cdot\left(\bar{Q}_{2}-\vec{Q}_{1}\right)}\left[\frac { \partial } { \partial \vec { \mathrm { P } } _ { 2 } } \frac { 1 } { ( 1 + L _ { + } ( i \vec { k } \cdot \vec { \mathrm { v } } _ { 1 } ) ) } \int _ { + } \frac { d \vec { \mathrm { p } } _ { 3 } } { \vec { \mathrm { k } } \cdot ( \overline { \mathrm { V } } _ { 1 } - \mathrm { v } _ { 3 } ) } \vec { \mathrm { k } } \cdot \left(\overrightarrow{\mathrm{D}}_{13} \mathrm{~F}_{2}\left(\mathrm{x}_{1}, \mathrm{x}_{2}\right) \mathrm{F}_{1}\left(\overrightarrow{\mathrm{p}}_{3}\right)\right.\right. \\
& \left.\left.\left.+n \frac{\partial \mathrm{F}_{2}\left(\mathrm{x}_{1}, \mathrm{x}_{2}\right)}{\partial \overrightarrow{\mathrm{P}}_{1}} \int \overline{\mathrm{f}}_{2}^{1}\left(\overrightarrow{\mathrm{p}}_{3}, \overrightarrow{\mathrm{p}}_{4}, \overrightarrow{\mathrm{k}}\right) \mathrm{d} \overrightarrow{\mathrm{p}}_{4}\right)\right]+(1 \rightarrow 2)\right\} \text {. }
\end{aligned}
$$

This is an equation for $\partial \mathrm{F}_{2} / \partial \mathrm{t}$ to first order in $\mathrm{g}$. It is complicated but will yield some interesting insights. The integral terms can be simplified somewhat by examining the equation for $\mathrm{f}_{2}^{1}$.

Let us rewrite (53) for a uniform plasma by using $F_{2}^{1}\left(x_{3}, x_{1} ; F_{1}\right)-f_{2}^{1}\left(x_{3}, x_{1}\right)$ and the

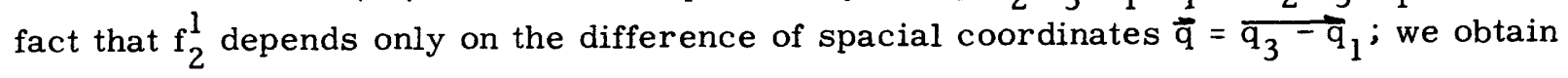

$$
\begin{aligned}
& \left.D_{o} f_{2}^{l}\left(\vec{p}_{3}, \vec{p}_{1}, \vec{q} ; F_{1}\right)+\frac{\left(\vec{p}_{3}-\vec{p}_{1}\right.}{m}\right) \cdot \frac{\partial}{\partial \vec{q}} f_{2}^{l}\left(\vec{p}_{3}, \vec{p}_{1}, \vec{q} ; F_{1}\right) \\
& +n \frac{\partial F_{1}\left(\vec{p}_{1}\right)}{\partial \vec{p}_{1}} \cdot \frac{\partial}{\partial \vec{q}} \int d \vec{p}_{5} d \vec{q}^{1} \psi\left(\vec{q}^{-1}\right) f_{2}^{1}\left(\vec{p}_{3}, \vec{p}_{5}, \vec{q}^{1} ; F_{1}\right) \\
& -n \frac{\partial F_{1}\left(\vec{p}_{2}\right)}{\partial \vec{p}_{3}} \cdot \frac{\partial}{\partial \vec{q}} \int d \vec{p}_{5} d \vec{q}^{1} \psi\left({\vec{q}-\vec{q}^{1}}^{1}\right) f_{2}^{1}\left(\vec{p}_{5}, \vec{p}_{1}, \vec{q}^{1}\right) \\
& =\frac{\partial}{\partial \overrightarrow{\mathrm{q}}} \psi(\overrightarrow{\mathrm{q}}) \cdot \mathrm{d}_{31} \mathrm{~F}_{1}\left(\overrightarrow{\mathrm{p}}_{3}\right) \mathrm{F}_{1}\left(\overrightarrow{\mathrm{p}}_{1}\right) .
\end{aligned}
$$

Again we introduce $f_{2}^{1}\left(\vec{p}_{3}, \bar{p}_{1}, \vec{q} ; S_{-\tau}^{1} F_{1}\right)$ and use the fact that $D_{0} \rightarrow \partial / \partial \tau$. We are again free to pick the value of $\tau$, since it is a dummy variable. However, since $\tau$ appears in the unknown function, its value must be selected carefully. We shall pick $\tau \rightarrow \infty$ but do so in the Laplace-transform space by letting the transform variable $\sigma \rightarrow 0$. This will also set $\partial \mathrm{f}_{2}^{1} / \partial \tau$ equal to zero, as it must since $\mathrm{D}_{\mathrm{O}} \mathrm{F}_{1}=0$. This technique can be used because of the fact that for

$$
\begin{aligned}
& \bar{g}(\sigma)=\int_{0}^{\infty} g(\tau) e^{-\sigma \tau} d \tau, \\
& \lim _{\sigma \rightarrow 0} \sigma \bar{g}(\sigma)=g(0)
\end{aligned}
$$


and

$$
\mathrm{f}_{2}^{1}\left(\overrightarrow{\mathrm{p}}_{3}, \overrightarrow{\mathrm{p}}_{1}, \overrightarrow{\mathrm{q}}_{;} \mathrm{S}_{-\tau}^{1} \mathrm{~F}_{1}\right) \underset{\tau \rightarrow 0}{\longrightarrow} \mathrm{f}_{2}^{1}\left(\overrightarrow{\mathrm{p}}_{3}, \overline{\mathrm{p}}_{1}, \overrightarrow{\mathrm{q}}_{\mathrm{F}_{1}}\right) .
$$

The initial condition term will also disappear in the limit $\sigma \rightarrow 0$.

Putting (98) into (97) and carrying out a Fourier transform in space as in (75), we have

$$
\begin{aligned}
\lim _{\epsilon \rightarrow 0}\left(\epsilon-i \vec{k} \cdot\left(\vec{v}_{3}-\vec{v}_{1}\right)\right) \bar{f}_{2}^{l}\left(\vec{p}_{3}, \vec{p}_{1}, \vec{k}\right) & +\frac{4 \pi n e^{2} i}{k^{2}} \vec{k} \cdot \frac{\partial F_{1}\left(\vec{p}_{3}\right)}{\partial \vec{p}_{3}} \int d \vec{p}_{5} \vec{f}_{2}^{l}\left(\vec{p}_{5}, \vec{p}_{1}, \vec{k}\right) \\
& -\frac{4 \pi n e^{2} i}{k^{2}} \vec{k} \cdot \frac{\partial F_{1}\left(\vec{p}_{1}\right)}{\partial \vec{p}_{1}} \int d \vec{p}_{5} \vec{f}_{2}^{1}\left(\vec{p}_{3}, \vec{p}_{5}, \vec{k}\right) \\
& =-\frac{4 \pi e^{2} i}{k^{2}} \vec{k} \cdot d_{31} F_{1}\left(\vec{p}_{3}\right) F_{1}\left(\vec{p}_{1}\right) .
\end{aligned}
$$

Dividing by $\left(\epsilon-\mathrm{i} \overrightarrow{\mathrm{k}} \cdot\left(\overrightarrow{\mathrm{v}}_{3}-\overrightarrow{\mathrm{v}}_{1}\right)\right)$ and then integrating over $\overrightarrow{\mathrm{p}}_{3}$ yields

$$
\begin{aligned}
\int \vec{f}_{2}^{1}\left(\vec{p}_{3}, \vec{p}_{1}, \vec{k}\right) d \vec{p}_{3}= & \lim _{\epsilon-0} \frac{4 \pi e^{2}}{k^{2}}\left\{n \int \frac{\vec{k} \cdot \frac{\partial F_{1}\left(\vec{p}_{3}\right)}{\partial \vec{p}_{3}}}{\vec{k} \cdot\left(\vec{v}_{3}-\vec{v}_{1}\right)+i \epsilon} d \vec{p}_{3} \int d \vec{p}_{5} \vec{f}_{2}^{-1}\left(\vec{p}_{5}, \vec{p}_{1}, \vec{k}\right)\right. \\
& \left.-n \vec{k} \cdot \frac{\partial F_{1}\left(\vec{p}_{1}\right)}{\partial \vec{p}_{1}} \int \frac{d \vec{p}_{5} d \vec{p}_{3} \bar{f}_{2}^{-1}\left(\vec{p}_{3}, \vec{p}_{5}, \vec{k}\right)}{\vec{k} \cdot\left(\vec{v}_{3}-\vec{v}_{1}\right)+i \epsilon}+\int \frac{\vec{k} \cdot \vec{d}_{31} F_{1}\left(\vec{p}_{3}\right) F_{1}\left(\vec{p}_{1}\right)}{\vec{k} \cdot\left(\vec{v}_{3}-\vec{v}_{1}\right)+i \epsilon} d \vec{p}_{3}\right\} .
\end{aligned}
$$

Rearranging terms and taking the limit $\epsilon \rightarrow 0$, we have

$$
\begin{aligned}
& \frac{m \omega_{p}^{2}}{k^{2}} \vec{k} \cdot \frac{\partial F_{1}\left(\vec{p}_{1}\right)}{\partial \vec{p}_{1}} \int_{+} \frac{\left(F_{1}\left(\vec{p}_{3}\right)+n \int f_{2}^{l}\left(\vec{p}_{3}, \vec{p}_{5}, \vec{k}\right) d \vec{p}_{5}\right) d p_{3}}{\vec{k} \cdot\left(\bar{v}_{1}^{-\vec{v}_{3}}\right)} \\
& \quad=n \int \vec{f}_{2}^{1}\left(\vec{p}_{3}, \vec{p}_{1}, \vec{k}\right) d \vec{p}_{3}+\frac{F_{1}\left(\vec{p}_{1}\right) L_{+}\left(i \vec{k} \cdot \vec{v}_{1}\right)}{1+L_{+}\left(i \vec{k} \cdot \vec{v}_{1}\right)} .
\end{aligned}
$$

We may use (100), together with $\vec{v}_{1}-\vec{v}_{1}$ and $\vec{v}_{2}-\vec{v}_{2}$, in (96) to obtain $\frac{\partial \mathrm{F}_{2}\left(\mathrm{x}_{1}, \mathrm{x}_{2}\right)}{\partial \mathrm{t}}=\left[\mathrm{H}_{2}^{\mathrm{o}}+\psi_{12} ; \mathrm{F}_{2}\left(\mathrm{x}_{1}, \mathrm{x}_{2}\right)\right]-\mathrm{g} \frac{4 \pi \mathrm{e}^{2} i}{(2 \pi)^{3}} \int \frac{\mathrm{d} \overrightarrow{\mathrm{k}}}{\mathrm{k}^{2}} \overrightarrow{\mathrm{k}} \cdot\left\{\frac{\partial}{\partial \overrightarrow{\mathrm{P}}_{1}}\left[\left(\mathrm{n} \int \overline{\mathrm{f}}_{2}^{1}\left(\overrightarrow{\mathrm{p}}_{3}, \overrightarrow{\mathrm{P}}_{1}, \overrightarrow{\mathrm{k}}\right) \mathrm{d} \overrightarrow{\mathrm{p}}_{3}\right.\right.\right.$ 


$$
\begin{aligned}
& \left.+\frac{F_{1}\left(\vec{P}_{1}\right) L_{+}\left(i \vec{k} \cdot \vec{V}_{1}\right)}{1+L_{+}\left(i \vec{k} \cdot \vec{V}_{1}\right)}\right) \frac{1}{\vec{k} \cdot \frac{\partial F_{1}\left(\vec{P}_{1}\right)}{\partial \vec{P}_{1}}} \cdot \frac{\partial F_{2}\left(x_{1}, x_{2}\right)}{\partial \vec{P}_{1}} \\
& \left.-\frac{F_{2}\left(x_{1}, x_{2}\right) L_{+}\left(i \vec{k} \cdot \vec{V}_{1}\right)}{1+L_{+}\left(i \vec{k} \cdot \vec{V}_{1}\right)}\right]+e^{i k \cdot\left(Q_{2}-Q_{1}\right)}\left[\left\{n \int f_{2}^{1}\left(\vec{p}_{3}, \vec{P}_{1}, \vec{k}\right) d \vec{p}_{3}\right.\right. \\
& \left.+\frac{F_{1}\left(\vec{P}_{1}\right) L_{+}\left(i \vec{k} \cdot \vec{V}_{1}\right)}{1+L_{+}\left(i \vec{k} \cdot \vec{V}_{1}\right)}\right\} \frac{1}{\vec{k} \cdot \frac{\partial F_{1}\left(\vec{P}_{1}\right)}{\partial \vec{P}_{1}} \frac{\partial}{\partial \vec{P}_{2}}\left(\vec{k} \cdot \frac{\partial}{\partial \vec{P}_{1}} F_{2}\left(x_{1}, x_{2}\right)\right)} \\
& \left.\left.-\frac{L_{+}\left(i \vec{k} \cdot \vec{V}_{1}\right)}{1+L_{+}\left(i \vec{k} \cdot \vec{V}_{1}\right)} \frac{\partial F_{2}\left(x_{1}, x_{2}\right)}{\partial \vec{P}_{2}}\right]+(1 \longleftrightarrow 2)\right\}
\end{aligned}
$$

This form of the equation for $\mathrm{F}_{2}$ is still very complex; however, with it we can easily obtain results to be compared with (17). Obtaining these results will entail making an adiabatic hypothesis for $F_{2}$ in the sense that we now assume that the time dependence of $\mathrm{F}_{2}$ resides within a functional dependence of $\mathrm{F}_{1}$.

In line with this hypothesis, we expand

$$
\mathrm{F}_{2}\left(\mathrm{x}_{1}, \mathrm{x}_{2} ; \mathrm{F}_{1}\right)=\mathrm{F}_{2}^{\mathrm{o}}\left(\mathrm{x}_{1}, \mathrm{x}_{2} ; \mathrm{F}_{1}\right)+\mathrm{gF}_{2}^{1}\left(\mathrm{x}_{1}, \mathrm{x}_{2} ; \mathrm{F}_{1}\right)+\ldots
$$

and introduce $D_{r}$ operators that replace $\partial F_{1} / \partial t$ with $A_{1}^{r}$ in (96) and then we equate equal powers of $\mathrm{g}$, for the first two powers, to obtain

$$
\mathrm{D}_{\mathrm{o}} \mathrm{F}_{2}^{\mathrm{o}}\left(\mathrm{x}_{1}, \mathrm{x}_{2} ; \mathrm{F}_{1}\right)=\left[\mathrm{H}_{2}^{\mathrm{O}}+\psi_{12} ; \mathrm{F}_{2}^{\mathrm{o}}\left(\mathrm{x}_{1}, \mathrm{x}_{2} ; \mathrm{F}_{1}\right)\right]
$$

and

$$
\begin{aligned}
& \mathrm{D}_{1} \mathrm{~F}_{2}^{\mathrm{O}}\left(\mathrm{x}_{1}, \mathrm{x}_{2} ; \mathrm{F}_{1}\right)+\mathrm{D}_{\mathrm{o}} \mathrm{F}_{2}^{\mathrm{l}}\left(\mathrm{x}_{1}, \mathrm{x}_{2} ; \mathrm{F}_{1}\right)=\left[\mathrm{H}_{2}^{\mathrm{O}}+\psi_{12} ; \mathrm{F}_{2}^{1}\left(\mathrm{x}_{1}, \mathrm{x}_{2} ; \mathrm{F}_{1}\right)\right] \\
& -\frac{4 \pi e^{2} i}{(2 \pi)^{3}} \int \frac{d \vec{k}}{k^{2}} \vec{k} \cdot\left\{\frac { \partial } { \partial \overline { \mathrm { P } } _ { 1 } } \left[\left\{n \int \overline{\mathrm{f}}_{2}^{l}\left(\overrightarrow{\mathrm{p}}_{3}, \overrightarrow{\mathrm{P}}_{1}, \overrightarrow{\mathrm{k}}\right) \mathrm{d} \overrightarrow{\mathrm{p}}_{3}\right.\right.\right. \\
& \left.+\frac{F_{1}\left(\vec{P}_{1}\right) L_{+}\left(i \vec{k} \cdot \vec{V}_{1}\right)}{1+L_{+}\left(i \vec{k} \cdot \vec{V}_{1}\right)}\right\} \frac{1}{\vec{k} \cdot \frac{\partial F_{1}\left(\vec{P}_{1}\right)}{\partial \vec{P}_{1}}} \vec{k} \cdot \frac{\partial F_{2}^{o}\left(x_{1}, x_{2}^{2}\right)}{\partial \vec{P}_{1}} \\
& \left.-\frac{F_{2}^{o}\left(x_{1}, x_{2}\right) L_{+}\left(i \vec{k} \cdot \vec{v}_{1}\right)}{1+L_{+}\left(i \vec{k} \cdot \vec{v}_{1}\right)}\right]+e^{i \vec{k} \cdot\left(\vec{Q}_{2}-\vec{Q}_{1}\right)}\left[\left\{n \int \vec{f}_{2}^{1}\left(\vec{p}_{3}, \vec{P}_{1}, \vec{k}\right) d \vec{p}_{3}\right.\right.
\end{aligned}
$$




$$
\begin{aligned}
& \left.+\frac{\mathrm{F}_{1}\left(\overrightarrow{\mathrm{P}}_{1}\right) \mathrm{L}_{+}\left(\mathrm{ik} \cdot \overrightarrow{\mathrm{V}}_{1}\right)}{1+\mathrm{L}_{+}\left(\mathrm{i} \overrightarrow{\mathrm{k}} \cdot \overrightarrow{\mathrm{V}}_{1}\right)}\right\} \frac{1}{\mathrm{k} \cdot \frac{\partial \mathrm{F}_{1}\left(\overrightarrow{\mathrm{P}}_{1}\right)}{\partial \overrightarrow{\mathrm{P}}_{1}} \frac{\partial}{\partial \overrightarrow{\mathrm{P}}_{2}}\left(\overrightarrow{\mathrm{k}} \cdot \frac{\partial}{\partial \overrightarrow{\mathrm{P}}_{1}} \mathrm{~F}_{2}^{\mathrm{o}}\left(\mathrm{x}_{1}, \mathrm{x}_{2}\right)\right)} \\
& \left.\left.-\frac{\mathrm{L}_{+}\left(\mathrm{ik} \cdot \overrightarrow{\mathrm{V}}_{1}\right)}{1+\mathrm{L}_{+}\left(\mathrm{i} \overrightarrow{\mathrm{k}} \cdot \overrightarrow{\mathrm{V}}_{1}\right)} \frac{\partial \mathrm{F}_{2}^{\mathrm{O}}\left(\mathrm{x}_{1}, \mathrm{x}_{2}\right)}{\partial \overrightarrow{\mathrm{P}}_{2}}\right]+(1 \longleftrightarrow 2)\right\} .
\end{aligned}
$$

Let us first discuss the solution to (102). By the same procedure that we used in section 3. 1 we can replace the $\mathrm{D}_{\mathrm{O}}$ operator and obtain

$$
\frac{\partial}{\partial t} F_{2}^{o}\left(x_{1}, x_{2} ; S_{-\tau}^{l} F_{1}\right)=\left[H_{2}^{o}+\psi_{12} ; F_{2}^{O}\left(x_{1}, x_{2} ; S_{-\tau}^{l} F_{1}\right)\right] \text {. }
$$

By (26) the solution to this is

$$
F_{2}^{O}\left(x_{1}, x_{2} ; S_{-\tau}^{l} F_{1}\right)=S_{-\tau}^{2} F_{2}^{O}\left(x_{1}, x_{2} ; F_{1}\right)
$$

or, letting $\mathrm{F}_{1} \rightarrow \mathrm{S}_{+\tau}^{\mathrm{l}} \mathrm{F}_{1}$ in the functional dependence, we have the solution

$$
F_{2}^{o}\left(x_{1}, x_{2} ; F_{1}\right)=S_{-\tau}^{2} F_{2}^{o}\left(x_{1}, x_{2} ; S_{\tau}^{l} F_{1}\right) \text {. }
$$

Since the left-hand side is independent of $\tau$, we may again pick the value of $\tau$ arbitrarily. We pick $\tau \rightarrow \infty$ and use the boundary condition that is similar to (49)

$$
F_{2}^{\circ}\left(x_{1}, x_{2} ; F_{1}\right) \underset{\left|q_{1}-q_{2}\right| \rightarrow \infty}{\longrightarrow} F_{1}\left(x_{1}\right) F_{1}\left(x_{2}\right) .
$$

Using these, we obtain

$$
\begin{aligned}
F_{2}^{O}\left(x_{1}, x_{2} ; F_{1}\right) & =\lim _{\tau \rightarrow \infty} S_{-\tau}^{2} \dot{S}_{\tau}^{2} F_{1}\left(x_{1}\right) F_{1}\left(x_{2}\right) \\
& =F_{1}\left(X_{1}\right) F_{1}\left(X_{2}\right) .
\end{aligned}
$$

For the homogeneous plasma

$$
F_{2}^{O}\left(x_{1}, x_{2} ; F_{1}\right)=F_{1}\left(\vec{P}_{1}\right) F_{1}\left(\vec{P}_{2}\right) \text {. }
$$

By the definition of the $D_{1}$ operator we have

$$
\begin{aligned}
& D_{1} F_{2}^{O}=D_{1} F_{1}\left(\vec{P}_{1}\right) F_{1}\left(\overrightarrow{\mathrm{P}}_{2}\right) \\
& =n F_{1}\left(\vec{P}_{2}\right) \int\left[\psi_{13}\left(\vec{Q}_{1}-\vec{q}_{3}\right) ; f_{2}^{1}\left(\vec{P}_{1}, \vec{p}_{3},{\overrightarrow{Q_{1}-q_{3}}}\right)\right] d x_{3} \\
& +\mathrm{nF}_{1}\left(\overrightarrow{\mathrm{P}}_{1}\right) \int\left[\Psi_{23}\left(\overrightarrow{\mathrm{Q}}_{2}-\mathrm{q}_{3}\right) ; \mathrm{f}_{2}^{1}\left(\overrightarrow{\mathrm{P}}_{2}, \overrightarrow{\mathrm{p}}_{3}, \overrightarrow{\mathrm{Q}_{2}-\mathrm{q}_{3}}\right)\right] \mathrm{dx} x_{3}
\end{aligned}
$$




$$
=-\frac{4 \pi \mathrm{e}^{2} \mathrm{i}}{(2 \pi)^{3}} \mathrm{~F}_{1}\left(\mathrm{P}_{2}\right) \int \frac{\mathrm{d} \overrightarrow{\mathrm{k}}}{\mathrm{k}^{2}} \overrightarrow{\mathrm{k}} \cdot \frac{\partial}{\partial \overrightarrow{\mathrm{P}}_{1}} \int \overline{\mathrm{f}}_{2}^{\mathrm{l}}\left(\overrightarrow{\mathrm{P}}_{1}, \overrightarrow{\mathrm{p}}_{3}, \overrightarrow{\mathrm{k}}\right) \mathrm{dp} \mathrm{p}_{3}+(1 \longleftrightarrow 2) .
$$

Upon inserting $F_{2}^{\circ}$ into (103), we see that (107) just cancels the two integral terms that have no $\left(\overrightarrow{Q_{1}-Q_{2}}\right)$-dependence. This cancellation is the plausible way of avoiding the secondary divergence, mentioned with (19), which results from an integration over $\vec{q}_{3}$. The subtraction of $D_{1} F_{2}^{o}\left(x_{1}, x_{2}\right)$ involves the difference of two integrals that are to be cut off. This removes the dependence on the cutoff distance in the equation for $\mathrm{D}_{\mathrm{O}} \mathrm{F}_{2}^{\mathrm{l}}$.

Thus (103) becomes

$$
\begin{aligned}
& \mathrm{D}_{\mathrm{O}} \mathrm{F}_{2}^{1}\left(\mathrm{x}_{1}, \mathrm{x}_{2} ; \mathrm{F}_{1}\right)=\left[\mathrm{H}_{2}^{\mathrm{O}}+\psi_{12} ; \mathrm{F}_{2}^{1}\left(\mathrm{x}_{1}, \mathrm{x}_{2} ; \mathrm{F}_{1}\right)\right] \\
& -n \frac{4 \pi e^{2}}{(2 \pi)^{3}} i \frac{\partial F_{1}\left(\vec{P}_{2}\right)}{\partial \vec{P}_{2}} \cdot \int \frac{d \vec{k}}{k^{2}} \vec{k} e^{i \vec{k} \cdot\left(\vec{Q}_{2}-\vec{Q}_{1}\right)} \int \vec{f}_{2}\left(\vec{p}_{3}, \vec{P}_{1}, \vec{k}\right) d \vec{p}_{3} \\
& -n \frac{4 \pi e^{2}}{(2 \pi)^{3}} i \frac{\partial F_{1}\left(\vec{P}_{1}\right)}{\partial \vec{P}_{1}} \cdot \int \frac{d \vec{k}}{k^{2}} \vec{k} e^{i \vec{k} \cdot\left(\vec{Q}_{1}-\vec{Q}_{2}\right)} \int \bar{f}_{2}^{l}\left(\vec{p}_{3}, \vec{P}_{2}, \vec{k}\right) d \vec{p}_{3} \\
& =\left[\frac{\mathrm{P}_{1}^{2}+\mathrm{P}_{2}^{2}}{2 \mathrm{~m}} ; \mathrm{F}_{2}^{1}\left(\mathrm{x}_{1}, \mathrm{x}_{2} ; \mathrm{F}_{1}\right)\right] \\
& +n \int\left[\psi_{13}\left(\vec{Q}_{1}-\vec{q}_{3}\right) ; F_{1}\left(\vec{P}_{1}\right) f_{2}^{1}\left(\vec{P}_{2}, \vec{p}_{3},{\overrightarrow{Q_{2}}-\mathrm{q}_{3}}\right)\right] d x_{3} \\
& +n \int\left[\psi_{23}\left(\overrightarrow{Q_{2}-q_{3}}\right) ; F_{1}\left(\vec{P}_{2}\right) f_{2}^{l}\left(\vec{P}_{1}, \vec{p}_{3},{\overrightarrow{Q_{1}}-q_{3}}\right)\right] d x_{3} \text {, }
\end{aligned}
$$

where, in the latter form, we have written the integrals in $\bar{q}$-space and used the fact that

$$
\mathrm{H}_{2}^{\mathrm{o}}+\psi_{12}=\frac{\mathrm{P}_{1}^{2}+\mathrm{P}_{2}}{2 \mathrm{~m}}
$$

Now add the term $\left[\psi_{12}\left(\overrightarrow{Q_{1}-Q_{2}}\right) ; F_{1}\left(\vec{P}_{1}\right) F_{1}\left(\vec{P}_{2}\right)\right]$ to and subtract it from the right-hand side of (108). By comparing the results with (97) and using the invariance of the Poisson brackets with respect to a contact transformation, we see that $F_{2}^{l}$ can be split into two functions

$$
F_{2}^{1}\left(x_{1}, x_{2} ; F_{1}\right)=f_{2}^{1}\left(\vec{P}_{1}, \vec{P}_{2} ; \vec{Q}_{1}-\vec{Q}_{2}\right)+h_{2}\left(x_{1}, x_{2} ; F_{1}\right)
$$

where $h_{2}$ is the solution of

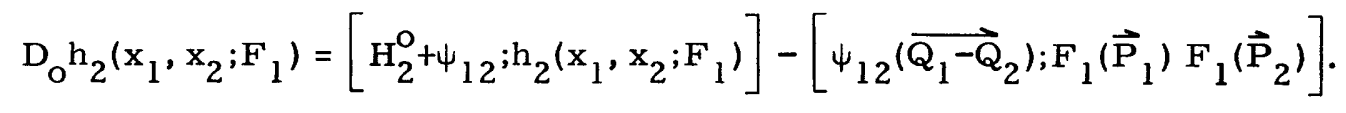


By the familiar use of the $D_{0}$ operators we can see that the solution to $(110)$ is

$$
h_{2}\left(x_{1}, x_{2} ; F_{1}\right)=-\left[\int_{0}^{\infty} d \tau \psi_{12}\left({\overrightarrow{Q_{1}-Q_{2}}}_{-}\left({\overrightarrow{V_{1}-V_{2}}}_{2}\right) ; F_{1}\left(\vec{P}_{1}\right) F_{1}\left(\vec{P}_{2}\right)\right]\right.
$$

Combining (106), (109), and (111), we obtain a final form for $F_{2}$ to first order in $g$ under the adiabatic hypothesis.

$$
\begin{aligned}
F_{2}\left(x_{1}, x_{2} ; F_{1}\right)= & F_{1}\left(\vec{P}_{1}\right) F_{1}\left(\vec{P}_{2}\right) \\
& \left.+f_{2}^{1}\left(\vec{P}_{1}, \vec{P}_{2},{\overrightarrow{Q_{1}-Q_{2}}}\right)-\left[\int_{0}^{\infty} d \tau \psi_{12}\left({\overrightarrow{Q_{1}-Q_{2}}}_{-\left(\bar{V}_{1}-\vec{V}_{2}\right.}\right) \tau\right) ; F_{1}\left(\vec{P}_{1}\right) F_{1}\left(\vec{P}_{2}\right)\right] .
\end{aligned}
$$

In Section IV we shall see that use of this function results in no divergence in the equation for $F_{1}$.

We shall look at (101) in its more general form, as a kinetic equation for $\mathrm{F}_{2}$. As such it is exact in $\psi_{12}$ and accurate to first order in all other interactions. If any collision term in the equation for $F_{1}$ is going to be used, it should be obtained from (101). For example, the expansion in $\mathrm{g}$ and the adiabatic hypothesis led to (112) and will be used in such a collision term.

We can also use (101) to study the limitations of making the adiabatic hypothesis for $F_{2}$. If in the solution we had expanded in $\mathrm{g}$ but not made the hypothesis, we would have had as a zero-order equation

$$
\frac{\partial \mathrm{F}_{2}^{\mathrm{O}}}{\partial \mathrm{t}}=\left[\mathrm{H}_{2}^{\mathrm{O}}+\psi_{12} ; \mathrm{F}_{2}^{\mathrm{O}}\right] \text {. }
$$

This is exactly the form of equation that Bogoliubov ${ }^{12}$ considers in showing that, for short-range forces, the adiabatic hypothesis is valid. He shows that, for forces of range $r_{o}, F_{2}^{O}\left(x_{1}, x_{2} ; t\right)$ would relax to the form $F_{2}^{o}\left(x_{1}, x_{2} ; F_{1}\right)$ in a time of the order $r_{o} / \bar{v}$. If for the long-range interaction we interpret a collision as described in section 1.2 , this would be a time of order

$$
\sqrt{\frac{\lambda_{D} e^{2}}{k T}} \frac{1}{\bar{v}}=\omega_{p}^{-1} \quad \sqrt{\frac{e^{2}}{k T \lambda_{D}}} \ll \omega_{p}^{-1},
$$

where $\omega_{p}^{-1}$ is the characteristic time of change of $F_{1}$. We can restate this by saying that an adiabatic hypothesis for $\mathrm{F}_{2}^{\mathrm{O}}$ is forced on us by the boundary conditions. To zero order in $\mathrm{g}$, the only force producing correlated motion is the two-body force, and the time that is such that this force is not of order $g$ is small compared with the time of change of $F_{1}$. (These statements for $F_{2}^{\circ}$ can be carried over immediately to the inhomogeneous case; but we shall not carry this out since the transition is not as simple for $\mathrm{F}_{2}^{1}$.)

If we use the value of $F_{2}^{\circ}$ thus obtained in the equation to first order, we have 


$$
\begin{aligned}
\frac{\partial F_{2}^{l}\left(x_{1}, x_{2}\right)}{\partial t}= & {\left[H_{2}^{o}+\psi_{12} ; F_{2}^{1}\left(x_{1}, x_{2}\right)\right] } \\
& +n \int\left[\psi_{13}\left(\overrightarrow{Q_{1}-\vec{q}_{3}}\right) ; F_{1}\left(\vec{P}_{1}\right) f_{2}^{l}\left(\vec{P}_{2}, \vec{p}_{3}, \overrightarrow{Q_{2}-q_{3}}\right)\right] d x_{3} \\
& +n \int\left[\psi_{23}\left(\vec{Q}_{2}-\vec{q}_{3}\right) ; F_{1}\left(\vec{P}_{2}\right) f_{2}^{l}\left(\vec{P}_{1}, \vec{p}_{3}, \overrightarrow{Q_{1}-q_{3}}\right)\right] d x_{3}
\end{aligned}
$$

Again an adiabatic hypothesis is attractive, since the whole source term is a functional of $F_{1}$. If we call this source term $\phi\left(x_{1}, x_{2} ; t\right)$, we can write the solution to (113) as

$$
\begin{aligned}
F_{2}^{1}\left(x_{1}, x_{2} ; t\right)= & s_{-\left(t-t_{0}\right)}^{2} F_{2}^{1}\left(x_{1}, x_{2} ; t_{0}\right) \\
& +\int_{t_{0}}^{t} s_{t^{\prime}-t}^{2} \phi\left(x_{1}, x_{2} ; t^{\prime}\right) d t^{\prime} \underset{t_{o} \rightarrow-\infty}{\longrightarrow} \int_{0}^{\infty} d t^{\prime} s_{-t^{\prime}}^{2} \phi\left(x_{1}, x_{2} ; t-t^{\prime}\right) .
\end{aligned}
$$

Here, we use the boundary condition that $\mathrm{F}_{2}^{\mathrm{l}}$ is zero at infinite separation, $\phi$ goes to zero for $\left|{\overrightarrow{q_{1}}-q_{2}}\right| \geqslant \lambda_{D}$, and the $t^{\prime}$-integration will back the particles off to this distance in a time $\sim \lambda_{D} / \bar{v}$. If $\phi$ has not changed in this time, we hold it constant throughout and say that

$$
F_{2}^{1}\left(x_{1}, x_{2} ; t\right)=\int_{0}^{\infty} d t^{\prime} s_{-t^{\prime}}^{2} \phi\left(x_{1}, x_{2} ; t\right) .
$$

Since $t$ in $\phi$ occurs only inside $F_{1},(114)$ is equivalent to the adiabatic hypothesis. The argument connected with (40) indicates that for a uniform plasma $F_{1}$ changes sufficiently slowly that these approximations in the integral of (114) are valid.

From this discussion we see that the adiabatic hypothesis depends on the order of g. To zero order it is well founded. To first order two problems enter. The first is the necessity of assuming a slow variation of $F_{1}$, as mentioned above. The second is that the hypothesis for $F_{2}^{1}\left(x_{1}, x_{2}\right)$ depends upon the hypothesis for $F_{2}^{1}\left(x_{1}, x_{i}\right)$ where $i \neq 2$. (The fact that the source term in (113) is a functional of $F_{1}$ depends on this.) Dupree ${ }^{11}$ has shown that the hypothesis for $F_{2}^{1}\left(x_{1}, x_{i}\right)$ depends also on the slow variation of $F_{1}$. It simply shows that the hypotheses for the various $F_{2}^{l}$ are interrelated and cannot be considered separately. The first-order effects in a plasma include shielding, which is a cooperative phenomenon. 


\section{DISCUSSION OF RESULTS}

In this section we discuss the result obtained in the previous section and see that it removes the divergence in (17), as desired. Section 4.1 will be devoted to a discussion of the equilibrium case as an example to increase the understanding of the results. Section 4.2 will contain a discussion of the more general case.

\section{1 THE EQUILIBRIUM CASE}

At equilibrium the momentum distributions are Maxwellian. Using the fact that $\overrightarrow{\mathrm{p}}$ and $\overline{\mathrm{P}}$ are related by the conservation of energy,

$$
\frac{\mathrm{P}_{1}^{2}+\mathrm{P}_{2}^{2}}{2 \mathrm{~m}}=\frac{\mathrm{p}_{1}^{2}+\mathrm{p}_{2}^{2}}{2 \mathrm{~m}}+\frac{\mathrm{e}^{2}}{\left|\overline{\mathrm{q}_{1}-\mathrm{q}_{2}}\right|}
$$

we may write the first term of (112) as

$$
F_{1}^{M}\left(P_{1}\right) F_{1}^{M}\left(P_{2}\right)=F_{1}^{M}\left(p_{1}\right) F_{1}^{M}\left(p_{2}\right) \exp \left[-\frac{e^{2}}{k T\left|\overline{q_{1}-q_{2}}\right|}\right]
$$

where $\mathbf{F}_{1}^{\mathbf{M}}(\mathrm{p})$ is the Maxwellian distribution.

Other authors $9,10,12$ have shown that for equilibrium the large-separation solution reduces to

$$
\mathrm{f}_{2}^{1}\left(\overline{\mathrm{p}}_{1}, \overline{\mathrm{p}}_{2}, \overline{\mathrm{q}_{1}-\mathrm{q}_{2}}\right)=-\frac{\mathrm{e}^{2}}{\mathrm{kT}\left|\overrightarrow{\mathrm{q}_{1}-\mathrm{q}_{2}}\right|} \exp \left[-\frac{\left|\overrightarrow{\mathrm{q}_{1}-\mathrm{q}_{2}}\right|}{\lambda_{D}}\right] \mathrm{F}_{1}^{M_{1}}\left(\mathrm{p}_{1}\right) \mathrm{F}_{1}^{M_{2}}\left(\mathrm{p}_{2}\right)
$$

In terms of the undeflected variables, and using (115), we obtain

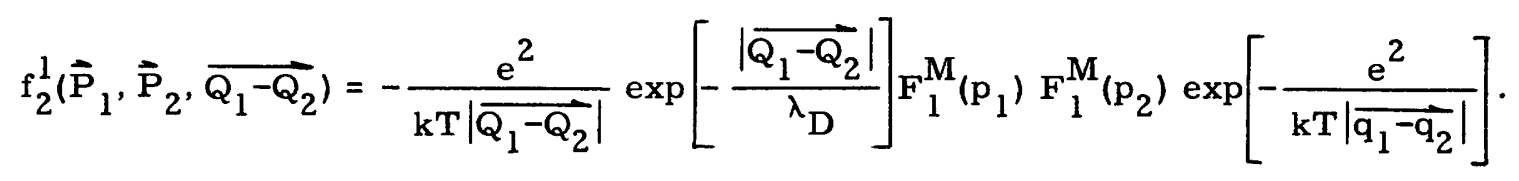

For equilibrium the final term in (112) may be evaluated as follows:

$$
\begin{aligned}
& {\left[\int_{0}^{\infty} \mathrm{d} \tau \psi_{12}\left(\overrightarrow{Q_{1}-Q_{2}}-\left(\overrightarrow{V_{1}-V_{2}}\right) \tau\right) ; F_{1}^{M}\left(P_{1}\right) F_{1}^{M}\left(P_{2}\right)\right]} \\
& \quad=-\frac{1}{k T} \frac{\partial}{\partial \vec{Q}_{1}} \int_{0}^{\infty} \mathrm{d} \tau \psi_{12}\left(\overrightarrow{Q_{1}-Q_{2}}-\left(\overrightarrow{V_{1}-V_{2}}\right) \tau\right) \cdot\left(\overrightarrow{V_{1}-\vec{V}_{2}}\right) F_{1}^{M}\left(P_{1}\right) F_{1}^{M}\left(P_{2}\right) \\
& \quad=\frac{1}{k T} \int_{0}^{\infty} \mathrm{d} \tau \frac{\partial}{\partial \tau} \psi_{12}\left(\overrightarrow{Q_{1}-Q_{2}}-\left(\overline{V_{1}-V_{2}}\right) \tau\right) F_{1}^{M}\left(P_{1}\right) F_{1}^{M}\left(P_{2}\right)
\end{aligned}
$$




$$
=-\frac{1}{k T\left|\overline{Q_{1}-Q_{2}}\right|} F_{1}^{M}\left(p_{1}\right) F_{1}^{M}\left(p_{2}\right) \exp \left[-\frac{e^{2}}{k T\left|\overline{q_{1}-q_{2}}\right|}\right] \text {. }
$$

The results (115), (117), and (118) give, for the equilibrium function $F_{2}^{e}\left(x_{1}, x_{2}\right)$,

$F_{2}^{e}\left(x_{1}, x_{2}\right)=F_{1}^{M}\left(p_{1}\right) F_{1}^{M}\left(p_{2}\right) \exp \left[-\frac{e^{2}}{k T\left|\overline{q_{1}-q_{2}}\right|}\right] \cdot\left\{1+\frac{e^{2}}{k T\left|\overline{Q_{1}-Q_{2}}\right|}\left(1-\exp \left[-\frac{\left|Q_{1}-Q_{2}\right|}{\lambda_{D}}\right]\right)\right\}$.

Those regions for which $\left|\overrightarrow{Q_{1}-Q_{2}}\right|$ differs from $\left|\overrightarrow{q_{1}-q_{2}}\right|$ are those for which $\left|\overrightarrow{Q_{1}-Q_{2}}\right| \ll \lambda_{D}$ and for which, therefore, the last term in parentheses is small. To the same order of $\mathrm{e}^{2} / \mathrm{kT} \lambda_{\mathrm{D}}$ we may replace $\left|\overrightarrow{\mathrm{Q}_{1}-\mathrm{Q}_{2}}\right|$ with $\left|\overrightarrow{\mathrm{q}_{1}-\mathrm{q}_{2}}\right|$ in (119). This substitution is consistent with our assumptions concerning the insensitivity of $F_{3}^{1}$ and $F_{2}^{1}$ to the difference between $\overline{\mathrm{Q}}$ and $\overrightarrow{\mathrm{q}}$.

We could approach the equilibrium case from a straightforward application of statistical mechanics. It has been shown ${ }^{1}$ that the effective potential field of a charge imbedded in a fluid of charged particles is approximately the Debye potential

$$
\frac{e^{2} e^{-r / \lambda} D}{r}
$$

If we were to assume that this is the potential energy between two charges, we would write the two-body distribution function as

$$
F_{2}^{e}\left(x_{1}, x_{2}\right)=F_{1}^{M}\left(p_{1}\right) F_{1}^{M}\left(p_{2}\right) \exp \left[-\frac{e^{2} \exp \left[-\frac{\left|\overline{q_{1}-q_{2}}\right|}{\lambda_{D}}\right]}{k T\left|\overline{q_{1}-q_{2}}\right|}\right] .
$$

If $\left|\widehat{q_{1}-q_{2}}\right| \gg e^{2} / k T$, we can expand the exponential and obtain the result corresponding to the large-separation solution, (116),

$$
F_{2}^{e}\left(x_{1}, x_{2}\right) \cong F_{1}^{M}\left(p_{1}\right) F_{1}^{M}\left(p_{2}\right)\left\{1-\frac{e^{2}}{k T\left|\overline{q_{1}-q_{2}}\right|} \exp \left[-\frac{\left|\overline{q_{1}-q_{2}}\right|}{\lambda_{D}}\right]\right\}
$$

If we do not wish to assume that $\left|\overline{\mathrm{q}_{1}-\mathrm{q}_{2}}\right| \gg \mathrm{e}^{2} / \mathrm{kT}$, we can write $\mathrm{F}_{2}^{\mathrm{e}}$ as

$$
F_{2}^{e}\left(x_{1}, x_{2}\right)=F_{1}^{M}\left(p_{1}\right) F_{1}^{M}\left(p_{2}\right) \exp \left[-\frac{e^{2}}{k T\left|\overline{q_{1}-q_{2}}\right|}\right] \exp \left[-\frac{e^{2}}{k T\left|\overline{q_{1}-q_{2}}\right|}\left(\exp \left[-\frac{\left|\overline{q_{1}-q_{2}}\right|}{\lambda_{D}}\right]-1\right)\right]
$$

and expand the exponential of

$$
\frac{e^{2}}{k T\left|\overline{q_{1}-q_{2}}\right|}\left(\exp \left[-\frac{\left|\overline{q_{1}-q_{2}}\right|}{\lambda_{D}}\right]-1\right)
$$


This function has its maximum at $\left|\overrightarrow{\mathrm{q}_{1}-\mathrm{q}_{2}}\right|=0$, the value at which it equals $\mathrm{e}^{2} / \mathrm{kT} \lambda_{\mathrm{D}}$, our small expansion parameter. Under this expansion we have

$F_{2}^{e}\left(x_{1}, x_{2}\right) \cong F_{1}^{M}\left(p_{1}\right) F_{1}^{M}\left(p_{2}\right) \exp \left[-\frac{e^{2}}{k T\left|\overline{q_{1}-q_{2}}\right|}\right] \cdot\left\{1-\frac{e^{2}}{k T\left|\overline{q_{1}-q_{2}}\right|}\left(\exp \left[-\frac{\left|\overline{q_{1}-q_{2}}\right|}{\lambda_{D}}\right]-1\right)\right\}$.

Equation 121 is (119) when we replace $\left|\overrightarrow{Q_{1}-Q_{2}}\right|$ with $\left|\overrightarrow{q_{1}-q_{2}}\right|$, and is the equilibrium function that we obtained by allowing for possible small values of $\left|\overline{q_{1}-q_{2}}\right|$. Both (119) and (121) result from expansions in the weakness of the forces giving rise to shielding.

If we regroup (121) as

$F_{2}^{e}=F_{1}^{M}\left(p_{1}\right) F_{1}^{M}\left(p_{2}\right)\left\{\exp \left[-\frac{e^{2}}{k T\left|\overline{q_{1}-q_{2}}\right|}\right]\left(1+\frac{e^{2}}{k T\left|\overline{q_{1}-q_{2}}\right|}\right)-\frac{e^{2}}{k T\left|\overline{q_{1}-q_{2}}\right|} \exp \left\lceil-\frac{\left|\overline{q_{1}-q_{2}}\right|}{\lambda_{D}} \mid\right\}\right.$

and expand for $\left|\overrightarrow{\mathrm{q}_{1}-\mathrm{q}_{2}}\right| \gg \mathrm{e}^{2} / \mathrm{kT}$, we obtain (120) to first order in $\mathrm{e}^{2} / \mathrm{kT}\left|\overrightarrow{\mathrm{q}_{1}-\mathrm{q}_{2}}\right|$. The effect of the additional function $\mathrm{h}_{2}\left(\mathrm{x}_{1}, \mathrm{x}_{2}\right)$ of section 3.2 in the transition to large separation is to cancel the first-order effect in the expansion of $F_{2}^{\circ}$. We shall see this in the more general case in section 4.2.

Another use for $\mathrm{F}_{2}\left(\mathrm{x}_{1}, \mathrm{x}_{2}\right)$ will be in the equation

$$
\frac{\partial \mathrm{F}_{1}\left(\mathrm{x}_{1}\right)}{\partial \mathrm{t}}=\left[\mathrm{H}_{1}^{\mathrm{o}} ; \mathrm{F}_{1}\left(\mathrm{x}_{1}\right)\right]+\mathrm{n} \int\left[\psi_{12} ; \mathrm{F}_{2}\left(\mathrm{x}_{1}, \mathrm{x}_{2}\right)\right] \mathrm{dx_{2 }} \text {. }
$$

The use of $\mathrm{F}_{2}\left(\mathrm{x}_{1}, \mathrm{x}_{2}\right)$ in the interaction term of (122) involves the removal of the distinction of particles 1 and 2, which was discussed at the beginning of section 2.3. The coordinate $\mathrm{x}_{2}$ in (122) is simply a dummy variable in the integration over a function that is considered correct for all values of $\left(\overline{q_{1}-q_{2}}\right)$.

We can use the expression (121) for $\mathrm{F}_{2}^{\mathrm{e}}$ in order to estimate the contribution of various terms in $F_{2}$ to the integral in (122). We know that the angular integrals over $\mathrm{F}_{2}^{\mathrm{e}}$ will give zero contribution, but we are justified in examining the $\left(\left|\overrightarrow{\mathrm{q}_{2}-\mathrm{q}_{1}}\right|\right)$-dependence for this case. In order to have convergence in the $\left(\left|\overrightarrow{q_{2}-q_{1}}\right|\right)$-integration, it is necessary to subtract the effect of the uniform background (also zero on the angular integration). Thus we shall examine the $\left(\left|\overline{q_{2}-q_{1}}\right|\right)$-dependence of the integral

$$
\int\left[\psi_{12} ; F_{2}^{e}\left(x_{1}, x_{2}\right)-F_{1}^{M}\left(p_{1}\right) F_{1}^{M}\left(p_{2}\right)\right] d x_{2}
$$

Our motivation for this is as follows. In the nonequilibrium case the angular integrations of (123) will not give zero. However, we can expect the $\left(\left|\overrightarrow{q_{2}-q_{1}}\right|\right)$-dependence to be roughly the same. If we can solve the equilibrium case, we can gain some insight into the nonequilibrium case.

Using (121) for $F_{2}^{e}$ in (123), we can write the $\left|\overrightarrow{q_{2}-q_{1}}\right|$ part of the $d x_{2}$-integration as 


$$
\int_{0}^{\infty} \mathrm{dr}\left[\exp \left[-\frac{\mathrm{e}^{2}}{\mathrm{kTr} r}\right]\left(1+\frac{\mathrm{e}^{2}}{\mathrm{kTr}}\right)-1-\frac{\mathrm{e}^{2}}{\mathrm{kTr}} \exp -\left[\frac{\mathrm{e}^{2}}{\mathrm{kTr}}-\frac{\mathrm{r}}{\lambda_{\mathrm{D}}}\right]\right] .
$$

Let us integrate the last term, 15

$$
\frac{\mathrm{e}^{2}}{\mathrm{kT}} \int_{0}^{\infty} \frac{\mathrm{dr}}{\mathrm{r}} \exp \left[-\frac{\mathrm{e}^{2}}{\mathrm{kTr}}-\frac{\mathrm{r}}{\lambda_{\mathrm{D}}}\right]=\frac{2 \mathrm{e}^{2}}{\mathrm{kT}} \mathrm{K}_{\mathrm{o}}\left(2 \sqrt{\frac{\mathrm{e}^{2}}{\mathrm{kT} \lambda_{\mathrm{D}}}}\right)
$$

We can expand the modified Bessel function ${ }^{16}$ for small argument and obtain

$$
\frac{\mathrm{e}^{2}}{\mathrm{kT}} \int_{0}^{\infty} \frac{\mathrm{dr}}{\mathrm{r}} \exp \left[-\frac{\mathrm{e}^{2}}{\mathrm{kTr}}-\frac{\mathrm{r}}{\lambda_{\mathrm{D}}}\right] \cong-\frac{\mathrm{e}^{2}}{\mathrm{kT}}\left(\ln \gamma \frac{\mathrm{e}^{2}}{\mathrm{kT} \lambda_{\mathrm{D}}}+0\left(\frac{\mathrm{e}^{2}}{\mathrm{kT} \lambda_{\mathrm{D}}} \ln \frac{\mathrm{e}^{2}}{\mathrm{kT} \lambda_{\mathrm{D}}}\right)\right),
$$

where $\gamma=e^{C}$, and $C$ is Euler's number, 0.577 .

Use of the function (121) has resulted in a convergent integral. Had we followed other authors and used (116), the integral would have diverged at small distances. Cutting it off at the distance of closest approach, $e^{2} / k T$, yields

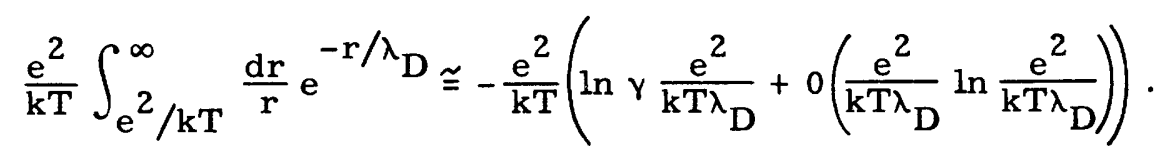

This result agrees with the more exact treatment to the same order of $e^{2} / k T \lambda_{D}$.

This surprising accuracy of the cutoff procedure occurs because, to a function that varies as $\lambda_{D}$, the function $\exp \left(-\mathrm{e}^{2} / \mathrm{kTr}\right.$ ) cuts off very sharply. Because this result was obtained only from the $\left(\left|\overrightarrow{q_{2}-q_{1}}\right|\right)$-dependence of the integral of (123) and did not rely on the symmetry of the equilibrium function, we can expect similar results for any nonequilibrium function $\mathrm{f}_{2}^{\mathrm{l}}\left(\overrightarrow{\mathrm{P}}_{1}, \overrightarrow{\mathrm{P}}_{2}, \overline{\mathrm{q}}_{1}-\mathrm{q}_{2}\right)$. We therefore expect that we can still retain accuracy to the same order in $\mathrm{g}$ if we set

$$
\mathrm{f}_{2}^{1}\left(\overline{\mathrm{P}}_{1}, \overline{\mathrm{P}}_{2}, \overline{\mathrm{Q}}_{1}-\overline{\mathrm{Q}}_{2}\right) \Longrightarrow \mathrm{f}_{2}^{1}\left(\overline{\mathrm{p}}_{1}, \overline{\mathrm{p}}_{2}, \overline{\mathrm{q}_{1}-\mathrm{q}_{2}}\right)
$$

and cut off the integration at $\mathrm{e}^{2} / \mathrm{kT}$.

The new results obtained by considerations of the close approach are in the first two terms of the integral in (124),

$$
\int_{0}^{\infty} d r\left[\exp \left[-\frac{\mathrm{e}^{2}}{\mathrm{kTr}}\right]\left(1+\frac{\mathrm{e}^{2}}{\mathrm{kTr}}\right)-1\right]=-\frac{\mathrm{e}^{2}}{\mathrm{kT}}
$$

This is of the same order of $g$ as (125), and thus we obtain a non-negligible contribution as a result of the close collisions. The generalization of (127) to the nonequilibrium case will not be as simple as the result for $f_{2}^{1},(126)$. The generalization of (127) depends very intimately on the nature of the functions for $\left|\overrightarrow{q_{2}-q_{1}}\right| \sim e^{2} / k T$, and will be investigated in the next section. 


\subsection{THE NONEQUILIBRIUM CASE}

The discussion relating to the general case is necessarily more complex, since we must deal with general expressions rather than evaluate integrals of known functions. We shall see that to first order in $\mathrm{g}$, the entire effect of the close collisions can be put into one term that is a generalization of the Boltzmann collision integral. To this will be added another accounting for the velocity dependence of the collective interaction.

Consider (99) for $\overline{\mathrm{f}}_{2}^{-1}\left(\overrightarrow{\mathrm{P}}_{1}, \overline{\mathrm{P}}_{2}, \overrightarrow{\mathrm{k}}\right)$,

$$
\begin{aligned}
& \overline{\mathrm{f}}_{2}^{-1}\left(\overline{\mathrm{P}}_{1}, \overline{\mathrm{P}}_{2}, \overline{\mathrm{k}}\right)=-\frac{4 \pi i \mathrm{e}^{2}}{\mathrm{k}^{2}} \frac{\overline{\mathrm{k}} \cdot \overline{\mathrm{D}}_{12} \mathrm{~F}_{1}\left(\overline{\mathrm{P}}_{1}\right) \mathrm{F}_{1}\left(\overline{\mathrm{P}}_{2}\right)}{\left(\epsilon-\mathrm{i} \overline{\mathrm{k}} \cdot\left(\overline{\mathrm{V}}_{1}-\mathrm{V}_{2}\right)\right)} \\
& -\frac{4 \pi n e^{2} i}{k^{2}\left(\epsilon-i \vec{k} \cdot\left(\bar{V}_{1}-\vec{V}_{2}\right)\right)}\left\{\vec{k} \cdot \frac{\partial F_{1}\left(\vec{P}_{1}\right)}{\partial \vec{P}_{1}} \int d \vec{p}_{3} \bar{f}_{2}^{1}\left(\vec{p}_{3}, \vec{P}, \vec{k}\right)\right. \\
& \left.-\bar{k} \cdot \frac{\partial F_{1}\left(\overrightarrow{\mathrm{P}}_{2}\right)}{\partial \overrightarrow{\mathrm{P}}_{2}} \int \mathrm{d} \overrightarrow{\mathrm{p}}_{3} \overline{\mathrm{f}}_{2}^{\mathrm{l}}\left(\overrightarrow{\mathrm{P}}_{1}, \overrightarrow{\mathrm{p}}_{3}, \overrightarrow{\mathrm{k}}\right)\right\} \text {. }
\end{aligned}
$$

In (128) we define

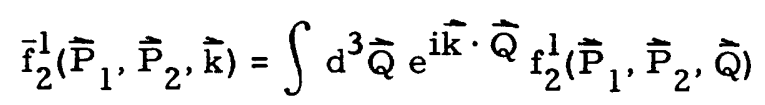

and

$$
-\frac{4 \pi i e^{2}}{k^{2}} \frac{\vec{k} \cdot \vec{D}_{12} F_{1}\left(\vec{P}_{1}\right) F_{1}\left(\vec{P}_{2}\right)}{\left(\epsilon-i \vec{k} \cdot\left(\bar{V}_{1}-\vec{V}_{2}\right)\right)}=\int d \bar{Q}_{12} e^{i \vec{k} \cdot \bar{Q}_{12}}\left[\int_{0}^{\infty} d \tau \psi_{12}\left(\vec{Q}_{12}-\left(\bar{V}_{1}-\vec{V}_{2}\right) \tau\right) ; F_{1}\left(\bar{P}_{1}\right) F_{1}\left(\vec{P}_{2}\right)\right] .
$$

From (130) we see that small distances, $\left|\overrightarrow{Q_{1}-Q_{2}}\right| \ll \lambda_{D}$, correspond to large wave numbers, $k \gg \lambda_{D}^{-1}$, in the region of chief contribution of the transform. For large $k$, (129) can be expanded in inverse powers of $k$ by using the first term of (129) as the first approximation in an iteration expansion,

$$
\begin{aligned}
& n \vec{f}_{2}^{l}\left(\overrightarrow{\mathrm{P}}_{1}, \overrightarrow{\mathrm{P}}_{2}, \overrightarrow{\mathrm{k}}\right)+\frac{4 \pi n \mathrm{e}^{2}}{\mathrm{k}^{2}} \mathrm{i} \frac{\overrightarrow{\mathrm{k}} \cdot \overline{\mathrm{D}}_{12} \mathrm{~F}_{1}\left(\overrightarrow{\mathrm{P}}_{1}\right) \mathrm{F}_{1}\left(\overline{\mathrm{P}}_{2}\right)}{\left(\epsilon-\mathrm{i} \overrightarrow{\mathrm{k}} \cdot\left(\overline{\mathrm{V}}_{1}-\overrightarrow{\mathrm{V}}_{2}\right)\right)} \\
& \cong \frac{\left(\frac{4 \pi n e^{2}}{{ }^{2}}\right)^{2} i}{\left(\epsilon-i \vec{k} \cdot\left(\overline{V_{1}-\vec{V}_{2}}\right)\right)}\left\{\vec{k} \cdot \frac{\partial F_{1}\left(\vec{P}_{1}\right)}{\partial \overline{\mathrm{P}}_{1}} \int_{+} d \bar{p}_{3} \frac{\vec{k} \cdot \vec{D}_{32} F_{1}\left(\vec{p}_{3}\right) F_{1}\left(\overrightarrow{\mathrm{P}}_{2}\right)}{\vec{k} \cdot\left(\overline{v_{3}-\vec{V}_{2}}\right)}\right.
\end{aligned}
$$




$$
\left.-\vec{k} \cdot \frac{\partial F_{1}\left(\bar{P}_{2}\right)}{\partial \vec{P}_{2}} \int_{-} d \bar{p}_{3} \frac{\vec{k} \cdot \bar{D}_{13} F_{1}\left(\bar{P}_{1}\right) F_{1}\left(\bar{P}_{2}\right)}{\vec{k} \cdot\left(\bar{V}_{1}-v_{3}\right)}\right\}+o\left(\frac{1}{\lambda_{D^{6}}^{6}}\right) .
$$

Successive terms fall off as $\mathrm{k}^{-2 \mathrm{n}}$.

For $k>\sqrt{k T / e^{2} \lambda_{D}}$ the right-hand side of $(131)$ is of order $\left(e^{2} / k T \lambda_{D}\right)^{2}$, which is of second-order smallness in $\mathrm{g}$. As was pointed out in section 1.2, the difference between $\bar{P}$ and $\bar{p}$ and $\bar{Q}$ and $\bar{q}$ becomes important only in the region $\left|\overrightarrow{q_{1}-q_{2}}\right|<$ $\sqrt{\mathrm{e}^{2} \lambda_{\mathrm{D}} / \mathrm{kT}}$. This fact implies that on the left-hand side of (131) we can replace $\bar{Q}$ with $\vec{q}$ and $\vec{P}$ with $\vec{p}$ and know that in those regions in which this replacement is not valid the function (131) is very small. Thus in $\vec{q}-$ space

$$
\begin{aligned}
& \mathrm{f}_{2}^{1}\left(\overrightarrow{\mathrm{P}}_{1}, \overrightarrow{\mathrm{P}}_{2}, \overline{\mathrm{Q}_{1}-\mathrm{Q}_{2}}\right)-\left[\int_{0}^{\infty} \mathrm{d} \tau \psi_{12}\left(\overline{\mathrm{Q}_{1}-\mathrm{Q}_{2}}-\left(\overline{\mathrm{V}_{1}-\mathrm{V}_{2}}\right) \tau\right) ; \mathrm{F}_{1}\left(\overline{\mathrm{P}}_{1}\right) \mathrm{F}_{1}\left(\overrightarrow{\mathrm{P}}_{2}\right)\right] \\
& \longrightarrow \mathrm{f}_{2}^{1}\left(\overline{\mathrm{p}}_{1}, \overline{\mathrm{p}}_{2}, \overline{\mathrm{q}_{1}-\mathrm{q}_{2}}\right)-\left[\int_{0}^{\infty} \mathrm{d} \tau \psi\left(\overline{\mathrm{q}_{1}-\mathrm{q}_{2}}-\left(\overline{\mathrm{v}_{1}-\mathrm{v}_{2}}\right) \tau\right) ; \mathrm{F}_{1}\left(\overrightarrow{\mathrm{p}}_{1}\right) \mathrm{F}_{1}\left(\overline{\mathrm{p}}_{2}\right)\right],
\end{aligned}
$$

where the error incurred will be of order $\left(e^{2} / k T \lambda_{D}\right)^{2}$. (The case of $\left|\overrightarrow{Q_{1}-Q_{2}}\right| \sim$ $\sqrt{\mathrm{e}^{2} \lambda_{\mathrm{D}} / \mathrm{kT}}$ will be mentioned later.)

The final use for $F_{2}\left(x_{1}, x_{2}\right)$ will be in the interaction term (I.T.) of the equation for $F_{1}$,

$$
\begin{aligned}
& \frac{\partial \mathrm{F}_{1}\left(\mathrm{x}_{1}\right)}{\partial \mathrm{t}}-\left[\mathrm{H}_{1}^{\mathrm{O}} ; \mathrm{F}_{1}\left(\mathrm{x}_{1}\right)\right] \equiv \mathrm{I} . \mathrm{T} . \\
& =\mathrm{n} \int\left[\Psi_{12} ;\left\{\mathrm{F}_{1}\left(\overrightarrow{\mathrm{P}}_{1}\right) \mathrm{F}_{1}\left(\overrightarrow{\mathrm{P}}_{2}\right)+\mathrm{f}_{2}^{1}\left(\overrightarrow{\mathrm{p}}_{1}, \overrightarrow{\mathrm{p}}_{2}, \overline{\mathrm{q}_{1}-\mathrm{q}_{2}}\right)\right.\right. \\
& \left.\left.\quad-\left[\int_{0}^{\infty} \mathrm{d} \tau \psi_{12}\left(\overline{\mathrm{q}_{1}-\mathrm{q}_{2}}-\left(\overline{\mathrm{v}_{1}-\mathrm{v}_{2}}\right) \tau\right) ; \mathrm{F}_{1}\left(\overrightarrow{\mathrm{p}}_{1}\right) \mathrm{F}_{1}\left(\overrightarrow{\mathrm{p}}_{2}\right)\right]\right\}\right] \mathrm{dx} \mathrm{x}_{2},
\end{aligned}
$$

where we have proved that there is no divergence for $\left|\overrightarrow{q_{1}-q_{2}}\right| \rightarrow 0$.

The third term of (133) appears to diverge for $\left|\overrightarrow{q_{1}-q_{2}}\right| \rightarrow \infty$, but we can easily show that this divergence is cancelled by the first term. Consider the equation for $F_{1}\left(\vec{P}_{1}\right) F_{1}\left(\vec{P}_{2}\right)$ in the region of small $\psi_{12}$, that is, large $\left|\overrightarrow{q_{1}-q_{2}}\right|$,

$$
\mathrm{D}_{\mathrm{o}} \mathrm{F}_{2}^{\mathrm{o}}\left(\mathrm{x}_{1}, \mathrm{x}_{2}\right)=\left[\mathrm{H}_{2}^{\mathrm{o}} ; \mathrm{F}_{2}^{\mathrm{o}}\left(\mathrm{x}_{1}, \mathrm{x}_{2}\right)\right]+\left[\Psi_{12} ; \mathrm{F}_{2}^{\mathrm{o}}\left(\mathrm{x}_{1}, \mathrm{x}_{2}\right)\right] \text {. }
$$

Using the usual solution methods and (26), we may obtain an expansion for small $\Psi_{12}$,

$$
\begin{aligned}
F_{1}\left(\vec{P}_{1}\right) F_{1}\left(\vec{P}_{2}\right)= & F_{1}\left(\vec{p}_{1}\right) F_{1}\left(\vec{p}_{2}\right) \\
& +\left[\int_{0}^{\infty} \Psi_{12}\left(\overline{q_{1}-q_{2}}-\left(\overline{v_{1}-v_{2}}\right) \tau\right) d \tau ; F_{1}\left(\vec{p}_{1}\right) F_{1}\left(\vec{p}_{2}\right)\right]
\end{aligned}
$$




$$
\begin{aligned}
& +\int_{0}^{\infty}\left[\psi_{12}\left(\overline{\mathrm{q}_{1}-\mathrm{q}_{2}}-\left(\overline{\mathrm{v}_{1}-\mathrm{v}_{2}}\right) \tau\right) ;\left[\int_{\tau}^{\infty} \mathrm{d} \tau^{\prime} \psi_{12}\left(\overline{\mathrm{q}_{1}-\mathrm{q}_{2}}-\left(\overline{\mathrm{v}_{1}-\mathrm{v}_{2}}\right) \tau^{\prime}\right) ; \mathrm{F}_{1}\left(\overrightarrow{\mathrm{p}}_{1}\right) \mathrm{F}_{1}\left(\overrightarrow{\mathrm{p}}_{2}\right)\right]\right] \mathrm{d} \tau \\
& +\ldots
\end{aligned}
$$

Using this expansion in (133) in the region of large $\left|\widehat{q_{1}-q_{2}}\right|$, we see that the second term of (134) cancels the divergent term of (133). The higher powers of $\psi_{12}$ in (134) will converge in the integral of (133) for $\left|\overrightarrow{\mathrm{q}_{1}-\mathrm{q}_{2}}\right| \rightarrow \infty$.

We see that (133), although a sufficient result, is in an inconvenient form because the final term in the interaction term cancels the divergence in $f_{2}^{1}$ as $\left|\overrightarrow{q_{1}-q_{2}}\right|-0$ and the divergence in $F_{2}^{O}$ as $\left|\overrightarrow{q_{1}-q_{2}}\right| \rightarrow \infty$. We shall obtain another form that is equivalent to (133) but that is more convenient in the kinetic equation for $F_{1}$.

In the Appendix we derive the result (17) corresponding to the $\mathrm{f}_{2}^{1}$ term in the inter action term of (133),

$$
\frac{16 \pi^{2} n \mathrm{e}^{4}}{(2 \pi)^{3}} \iint \frac{\mathrm{d} \overrightarrow{\mathrm{k}} \mathrm{d} \overrightarrow{\mathrm{p}}_{2}}{\mathrm{k}^{4}} \overrightarrow{\mathrm{k}} \cdot \frac{\partial}{\partial \overrightarrow{\mathrm{p}}_{1}} \frac{\delta\left(\overrightarrow{\mathrm{k}} \cdot\left(\overline{\mathrm{v}}_{1}-\overrightarrow{\mathrm{v}}_{2}\right)\right)}{\left|1+\mathrm{L}_{+}\left(\mathrm{i} \overrightarrow{\mathrm{k}} \cdot \overline{\mathrm{v}}_{1}\right)\right|^{2}} \overrightarrow{\mathrm{k}} \cdot \overrightarrow{\mathrm{d}}_{12} \mathrm{~F}_{1}\left(\overrightarrow{\mathrm{p}}_{1}\right) \mathrm{F}_{1}\left(\overrightarrow{\mathrm{p}}_{2}\right)
$$

The corresponding expression for the last term in (133) is obtained by expressing the integral in $\overrightarrow{\mathrm{k}}$-space and is given by

$$
\frac{16 \pi^{2} n e^{4}}{(2 \pi)^{3}} \iint \frac{d \vec{k} d \vec{p}_{2}}{k^{4}} \vec{k} \cdot \frac{\partial}{\partial \vec{p}_{1}} \delta\left(\vec{k} \cdot\left(\vec{v}_{2}-\vec{v}_{1}\right)\right) \vec{k} \cdot \vec{d}_{12} F_{1}\left(\vec{p}_{1}\right) F_{1}\left(\vec{p}_{2}\right)
$$

(Using the fact that $\mathrm{L}_{+}(\mathrm{i} \overline{\mathrm{k}} \cdot \overrightarrow{\mathrm{v}}) \underset{\mathrm{k} \rightarrow \infty}{\longrightarrow} 0$, we can easily see that the difference between (135) and (136) converges for large $|\vec{k}|$ in agreement with (132).)

In the interaction term of (133) add and subtract the term

$$
\begin{aligned}
& \frac{16 \pi^{2} n \mathrm{e}^{4}}{(2 \pi)^{3}} \iint \frac{\mathrm{d} \overrightarrow{\mathrm{k}} \mathrm{d} \overline{\mathrm{p}}_{2}}{\left(\mathrm{k}^{2}+\mathrm{k}_{\mathrm{D}}^{2}\right)^{2}} \overrightarrow{\mathrm{k}} \cdot \frac{\partial}{\partial \overline{\mathrm{p}}_{1}} \delta\left(\overrightarrow{\mathrm{k}} \cdot\left(\overline{\mathrm{v}_{1}-\mathrm{v}_{2}}\right)\right) \overrightarrow{\mathrm{k}} \cdot \overrightarrow{\mathrm{d}}_{12} \mathrm{~F}_{1}\left(\overrightarrow{\mathrm{p}}_{1}\right) \mathrm{F}_{1}\left(\overrightarrow{\mathrm{p}}_{2}\right) \\
& \quad=\mathrm{n} \int\left[\psi_{12}^{\mathrm{D}} ;\left[\int_{0}^{\infty} \mathrm{d} \tau \psi_{12}^{\mathrm{D}}\left(\overline{\mathrm{q}_{1}-\mathrm{q}_{2}}-\left(\overline{\mathrm{v}_{1}-\mathrm{v}_{2}}\right) \tau\right) ; \mathrm{F}_{1}\left(\overrightarrow{\mathrm{p}}_{1}\right) \mathrm{F}_{1}\left(\overrightarrow{\mathrm{p}_{2}}\right)\right]\right] \mathrm{dx_{2 }},
\end{aligned}
$$

where

$$
\mathrm{k}_{\mathrm{D}} \equiv \lambda_{\mathrm{D}}^{-1}
$$

and

$$
\psi_{12}^{\mathrm{D}} \equiv \frac{\mathrm{e}^{2}}{\left|\overrightarrow{\mathrm{q}_{1}-\mathrm{q}_{2}}\right|} \exp \left[-\frac{\left|\overrightarrow{\mathrm{q}_{1}-\mathrm{q}_{2}}\right|}{\lambda_{\mathrm{D}}}\right]
$$


The difference between (135) and (137) also converges for $|\vec{k}| \rightarrow \infty$.

With these additions, the interaction term becomes

$$
\begin{aligned}
& \text { I. T. }=n \int\left[\psi_{12} ; F_{1}\left(\vec{P}_{1}\right) F_{1}\left(\vec{P}_{2}\right)-\left[\int_{0}^{\infty} \psi_{12}\left(\overline{q_{1}-q_{2}}-\left(\overline{v_{1}-v_{2}}\right) \tau\right) ; F_{1}\left(\vec{p}_{1}\right) F_{1}\left(\vec{p}_{2}\right)\right]\right] d x_{2} \\
& +n \int\left[\psi_{12}^{D} ;\left[\int_{0}^{\infty} d \tau \psi_{12}^{D}\left(\overline{q_{1}-q_{2}}-\left(\overline{v_{1}-v_{2}}\right) \tau\right) ; F_{1}\left(\vec{p}_{1}\right) F_{1}\left(\overrightarrow{p_{2}}\right)\right]\right] d x_{2} \\
& +n \int\left\{\left[\psi_{12} ; f_{2}^{l}\left(\bar{p}_{1}, \vec{p}_{2}, \overline{q_{1}-q_{2}}\right)\right]\right. \\
& \left.-\left[\psi_{12}^{D} ;\left[\int_{0}^{\infty} \mathrm{d} \tau \psi_{12}^{\mathrm{D}}\left(\overline{\mathrm{q}_{1}-\mathrm{q}_{2}}-\left(\overline{\mathrm{v}_{1} \rightarrow \mathrm{v}_{2}}\right) \tau\right) ; \mathrm{F}_{1}\left(\overline{\mathrm{p}}_{1}\right) \mathrm{F}_{1}\left(\overline{\mathrm{p}_{2}}\right)\right]\right]\right\} \mathrm{dx} \mathrm{x}_{2} \text {. }
\end{aligned}
$$

In order to combine the first three terms of (138), consider the equation for $F_{1}\left(\overrightarrow{\mathrm{P}}_{1}^{\mathrm{D}}\right) \mathrm{F}_{1}\left(\overrightarrow{\mathrm{P}}_{2}^{\mathrm{D}}\right)$ where

$$
\begin{aligned}
& \overrightarrow{\mathrm{P}}_{1}^{\mathrm{D}}=\lim _{\tau \rightarrow \infty} \mathrm{S}_{-\tau}^{\mathrm{D}} \overrightarrow{\mathrm{P}}_{1}, \\
& \overrightarrow{\mathrm{P}}_{2}^{\mathrm{D}}=\lim _{\tau \rightarrow \infty} S_{-\tau}^{\mathrm{D}} \overline{\mathrm{P}}_{2},
\end{aligned}
$$

and

$$
\frac{\partial}{\partial \tau} S_{-\tau}^{D} \phi\left(x_{1}, x_{2}\right)=\left[H_{2}^{o}+\psi_{12}^{D} ; S_{-\tau}^{D} \phi\left(x_{1}, x_{2}\right)\right]
$$

for any $\phi\left(x_{1}, x_{2}\right)$. Here, $S_{-\tau}^{D}$ is the operator that projects the particles 1 and 2 back along trajectories corresponding to a Debye-shielded collision.

By the familiar means, we have

$$
\begin{aligned}
D_{0} F_{1}\left(\overrightarrow{\mathrm{P}}_{1}^{\mathrm{D}}\right) \mathrm{F}_{1}\left(\overrightarrow{\mathrm{P}}_{2}^{\mathrm{D}}\right)= & {\left[\mathrm{H}_{2}^{\mathrm{O}}+\psi_{12}^{\mathrm{D}} ; \mathrm{F}_{1}\left(\overrightarrow{\mathrm{P}}_{1}^{\mathrm{D}}\right) \mathrm{F}_{1}\left(\overrightarrow{\mathrm{P}}_{2}^{\mathrm{D}}\right)\right] } \\
= & {\left[\mathrm{H}_{2}^{\mathrm{o}}+\psi_{12} ; \mathrm{F}_{1}\left(\mathrm{P}_{1}^{\mathrm{D}}\right) \mathrm{F}_{1}\left(\mathrm{P}_{2}^{\mathrm{D}}\right)\right] } \\
& +\left[\psi_{12}^{\mathrm{D}}-\psi_{12} ; \mathrm{F}_{1}\left(\mathrm{P}_{1}^{\mathrm{D}}\right) \mathrm{F}_{1}\left(\mathrm{P}_{2}^{\mathrm{D}}\right)\right] .
\end{aligned}
$$

Since

$$
\left|\psi_{12}^{D}-\psi_{12}\right| \leqslant e^{2} / \lambda_{D}
$$

we can expand $F_{1}\left(\vec{P}_{1}^{D}\right) F_{1}\left(\vec{P}_{2}^{D}\right)$, using $(26)$, in terms corresponding to powers of $\left(\mathrm{e}^{2} / \mathrm{kT} \lambda_{\mathrm{D}}\right)$,

$F_{1}\left(\overrightarrow{\mathrm{P}}_{1}^{\mathrm{D}}\right) \mathrm{F}_{1}\left(\overrightarrow{\mathrm{P}}_{2}^{\mathrm{D}}\right) \cong \mathrm{F}_{1}\left(\overrightarrow{\mathrm{P}}_{1}\right) \mathrm{F}_{1}\left(\overrightarrow{\mathrm{P}}_{2}\right)+\int_{0}^{\infty} \mathrm{d} \tau \mathrm{S}_{-\tau}^{2}\left[\psi_{12} \mathrm{D}_{12} ; \mathrm{F}_{1}\left(\overline{\mathrm{P}}_{1}\right) \mathrm{F}_{1}\left(\overrightarrow{\mathrm{P}}_{2}\right)\right]$. 
The difference $\psi_{12}^{D}-\psi_{12}$ is very small until the $\tau$-integration backs the particles to a separation, $\sim \lambda_{D}$ ' so that we obtain in the integral of (141) only the asymptotic velocities,

$$
\left[\int_{0}^{\infty} \mathrm{d} \tau\left\{\psi_{12}^{\mathrm{D}}\left(\overrightarrow{\mathrm{Q}_{1}-\mathrm{Q}_{2}}-\left(\overrightarrow{\mathrm{V}_{1}-\mathrm{V}_{2}}\right) \tau\right)-\psi_{12}\left(\overrightarrow{\mathrm{Q}_{1}-\mathrm{Q}_{2}}-\left(\overrightarrow{\mathrm{V}_{1}-\mathrm{V}_{2}}\right) \tau\right)\right\} ; \mathrm{F}_{1}\left(\overrightarrow{\mathrm{P}}_{1}\right) \mathrm{F}_{1}\left(\overrightarrow{\mathrm{P}}_{2}\right)\right] .
$$

As in (132), this difference is small unless $\left|\overrightarrow{\mathrm{q}_{1}-\mathrm{q}_{2}}\right| \geqslant \lambda_{\mathrm{D}}$, in which case $\overrightarrow{\mathrm{Q}} \rightarrow \overrightarrow{\mathrm{q}}$ and $\overrightarrow{\mathrm{P}} \rightarrow \overrightarrow{\mathrm{p}}$ $\left(\left|\overrightarrow{Q_{1}-Q_{2}}\right| \sim \lambda_{D}\right.$ will be discussed later $)$. Thus (141) becomes

$F_{1}\left(\overrightarrow{\mathrm{P}}_{1}^{\mathrm{D}}\right) \mathrm{F}_{1}\left(\overrightarrow{\mathrm{P}}_{2}^{\mathrm{D}}\right) \cong \mathrm{F}_{1}\left(\overrightarrow{\mathrm{P}}_{1}\right) \mathrm{F}_{1}\left(\overrightarrow{\mathrm{P}}_{2}\right)$

$$
+\left[\int_{0}^{\infty} \mathrm{d} \tau\left\{\psi_{12}^{\mathrm{D}}\left(\overrightarrow{\mathrm{q}_{1}-\mathrm{q}_{2}}-\left(\overline{\mathrm{v}_{1}-\mathrm{v}_{2}}\right) \tau\right)-\psi_{12}\left(\overline{\mathrm{q}_{1}-\mathrm{q}_{2}}-\left(\overrightarrow{\mathrm{v}_{1}-\mathrm{v}_{2}}\right) \tau\right)\right\} ; \mathrm{F}_{1}\left(\overrightarrow{\mathrm{p}}_{1}\right) \mathrm{F}_{1}\left(\overrightarrow{\mathrm{p}_{2}}\right)\right] \text {. }
$$

Using (143), we can obtain

$$
\begin{aligned}
\int\left[\psi_{12} ; \mathrm{F}_{1}\left(\overrightarrow{\mathrm{P}_{1}}\right) \mathrm{F}_{1}\left(\overrightarrow{\mathrm{P}_{2}}\right)\right] \mathrm{dx} \mathrm{x}_{2} \cong & \left\{\left[\psi_{12}^{\mathrm{D}} ; \mathrm{F}_{1}\left(\overrightarrow{\mathrm{P}}_{1}^{\mathrm{D}}\right) \mathrm{F}_{1}\left(\overrightarrow{\mathrm{P}}_{2}^{\mathrm{D}}\right)\right]\right. \\
& +\left[\psi_{12} ;\left[\int_{0}^{\infty} \mathrm{d} \tau \psi_{12}\left(\overline{\mathrm{q}_{1}-\mathrm{q}_{2}}-\left(\overline{\mathrm{v}_{1}-\mathrm{v}_{2}}\right) \tau\right) ; \mathrm{F}_{1}\left(\overrightarrow{\mathrm{p}_{1}}\right) \mathrm{F}_{1}\left(\overrightarrow{\mathrm{p}_{2}}\right)\right]\right] \\
& \left.-\left[\psi_{12}^{\mathrm{D}} ;\left[\int_{0}^{\infty} \mathrm{d} \tau \psi_{12}^{\mathrm{D}}\left(\overline{\mathrm{q}_{1}-\mathrm{q}_{2}}-\left(\overline{\mathrm{v}_{1}-\mathrm{v}_{2}}\right) \tau\right) ; \mathrm{F}_{1}\left(\overrightarrow{\mathrm{p}_{1}}\right) \mathrm{F}_{1}\left(\overrightarrow{\mathrm{p}_{2}}\right)\right]\right]\right\} \mathrm{dx} \mathrm{x}_{2}
\end{aligned}
$$

where the equality holds to first order in $\left(e^{2} / \mathrm{kT} \lambda_{D}\right)$. Using (144) in (138), we have

$$
\text { I. } \begin{aligned}
T .= & n \int\left[\psi_{12}^{\mathrm{D}} ; \mathrm{F}_{1}\left(\overrightarrow{\mathrm{P}}_{1}^{\mathrm{D}}\right) \mathrm{F}_{1}\left(\overline{\mathrm{P}}_{2}^{\mathrm{D}}\right)\right] \mathrm{dx_{2 }} \\
& +\int\left\{\left[\psi_{12} ; \mathrm{f}_{2}^{\mathrm{l}}\left(\overrightarrow{\mathrm{p}}_{1}, \overrightarrow{\mathrm{p}}_{2}, \overline{\mathrm{q}_{1}-\mathrm{q}_{2}}\right)\right]\right. \\
& \left.-\left[\psi_{12}^{\mathrm{D}} ;\left[\int_{0}^{\infty} \mathrm{d} \tau \psi_{12}^{\mathrm{D}}\left(\overline{\mathrm{q}_{1}-\mathrm{q}_{2}}-\left(\overline{\mathrm{v}_{1}-\mathrm{v}_{2}}\right) \tau\right) ; \mathrm{F}_{1}\left(\overrightarrow{\mathrm{p}}_{1}\right) \mathrm{F}_{1}\left(\overrightarrow{\mathrm{p}}_{2}\right)\right]\right]\right\} \mathrm{dx} \mathrm{x}_{2} .
\end{aligned}
$$

We may see from the following considerations that the first term of (145) is a Boltzmann collision integral with a Debye-shielded interaction. The method used is due to Bogoliubov. ${ }^{12}$

From the definition of $\overline{\mathrm{P}}^{\mathrm{D}}$

$$
\left[\mathrm{H}_{2}^{o}+\psi_{12}^{D} ; \mathrm{F}_{1}\left(\overline{\mathrm{P}}_{1}^{\mathrm{D}}\right) \mathrm{F}_{1}\left(\overline{\mathrm{P}}_{2}^{\mathrm{D}}\right)\right]=0 \text {. }
$$

Thus we set

$$
\int\left[\psi_{12}^{\mathrm{D}} ; \mathrm{F}_{1}\left(\overrightarrow{\mathrm{P}}_{1}^{\mathrm{D}}\right) \mathrm{F}_{1}\left(\overrightarrow{\mathrm{P}}_{2}^{\mathrm{D}}\right)\right] \mathrm{dx_{2 }}=\iint \frac{\left(\widehat{\mathrm{p}_{2}-\overrightarrow{\mathrm{p}}_{1}}\right)}{\mathrm{m}} \cdot \frac{\partial}{\partial \dot{\mathrm{q}_{2}}} \mathrm{~F}_{1}\left(\overrightarrow{\mathrm{P}}_{1}^{\mathrm{D}}\right) \mathrm{F}_{1}\left(\overrightarrow{\mathrm{P}}_{2}^{\mathrm{D}}\right) d \overrightarrow{\mathrm{p}}_{2} \mathrm{~d} \overrightarrow{\mathrm{q}}_{2}
$$


and recognize that $\overrightarrow{\mathrm{P}}_{i}^{\mathrm{D}}$ depends on $\overrightarrow{\mathrm{q}}_{2}$ only through $\overrightarrow{\mathrm{q}}=\overline{\mathrm{q}_{2}-\mathrm{q}_{1}}$. For the $\overrightarrow{\mathrm{q}}$-integration set up a cylindrical coordinate system with the positive $z$-axis parallel to $\overrightarrow{v_{2}-v_{1}}$. Denote the radius and polar angle by $a$ and $\phi$, respectively. Then

$$
\begin{aligned}
& \int \frac{\left(\overline{\mathrm{p}_{2}-\mathrm{p}_{1}}\right)}{\mathrm{m}} \cdot \frac{\partial}{\partial \overrightarrow{\mathrm{q}}} \mathrm{F}_{1}\left(\mathrm{P}_{1}^{\mathrm{D}}\right) \mathrm{F}_{1}\left(\mathrm{P}_{2}^{\mathrm{D}}\right) \mathrm{d} \overrightarrow{\mathrm{q}} \\
& \quad=\left|\frac{\overline{\mathrm{p}_{2}-\mathrm{p}_{1}}}{\mathrm{~m}}\right| \int_{0}^{2 \pi} \mathrm{d} \phi \int_{0}^{\infty} \mathrm{da} a \int_{-\infty}^{\infty} \mathrm{d} \xi \frac{\partial}{\partial \xi} \mathrm{F}_{1}\left(\mathrm{P}_{1}^{\mathrm{D}}\right) \mathrm{F}_{1}\left(\mathrm{P}_{2}^{\mathrm{D}}\right) .
\end{aligned}
$$

From the two-body problem under consideration, $\overrightarrow{\mathrm{P}}_{1}^{\mathrm{D}}\left(\mathrm{x}_{1}, \mathrm{x}_{2}\right)$ and $\overrightarrow{\mathrm{P}}_{2}^{\mathrm{D}}\left(\mathrm{x}_{1}, \mathrm{x}_{2}\right)$ are the precollision momenta that will yield the state $x_{1}, x_{2}$. From this definition

$$
\begin{array}{ll}
\left.\mathrm{P}_{1}^{\mathrm{D}}\left(\mathrm{x}_{1}, \mathrm{x}_{2}\right)\right|_{\xi=-\infty}=\overrightarrow{\mathrm{p}}_{1} & \left.\mathrm{P}_{2}^{\mathrm{D}}\left(\mathrm{x}_{1}, \mathrm{x}_{2}\right)\right|_{\xi=-\infty}=\overrightarrow{\mathrm{p}}_{2} \\
\left.\mathrm{P}_{1}^{\mathrm{D}}\left(\mathrm{x}_{1}, \mathrm{x}_{2}\right)\right|_{\xi=+\infty}=\overrightarrow{\mathrm{p}}_{1}^{*} & \left.\mathrm{P}_{2}^{\mathrm{D}}\left(\mathrm{x}_{1}, \mathrm{x}_{2}\right)\right|_{\xi=+\infty}=\overrightarrow{\mathrm{p}}_{2}^{*}
\end{array}
$$

where $\overrightarrow{\mathrm{p}}_{1}^{*}$ and $\overrightarrow{\mathrm{p}}_{2}^{*}$ are those momenta that, as precollision momenta, will give $\overrightarrow{\mathrm{p}}_{1}$ and $\overrightarrow{\mathrm{p}}_{2}$ as a final momentum. For coordinates $x_{1}$ and $x_{2}$ which are such that an interaction is in progress, $\overrightarrow{\mathrm{P}}_{i}^{\mathrm{D}}$ and $\overrightarrow{\mathrm{p}}_{i}^{*}$ are not equal. Only when the particles are separated by a distance that is much greater than $\lambda_{D}$ do $\overrightarrow{\mathrm{P}}_{i}^{\mathrm{D}}$ and $\overrightarrow{\mathrm{p}}_{i}^{*}$ become equal.

Using (146)-(148) in (145), we have

$$
\begin{aligned}
& \mathrm{n} \int\left[\psi_{12}^{\mathrm{D}} ; \mathrm{F}_{1}\left(\mathrm{P}_{1}^{\mathrm{D}}\right) \mathrm{F}_{1}\left(\mathrm{P}_{2}^{\mathrm{D}}\right)\right] \mathrm{dx} \mathrm{x}_{2} \\
& \quad=\mathrm{n} \int_{0}^{2 \pi} \int_{0}^{\infty} \int_{\left(\overrightarrow{\mathrm{p}_{2}}\right)}\left|\frac{\overrightarrow{\mathrm{p}_{2}-\mathrm{p}_{1}}}{\mathrm{~m}}\right|\left(\mathrm{F}_{1}\left(\overrightarrow{\mathrm{p}}_{1}^{*}\right) \mathrm{F}_{1}\left(\overrightarrow{\mathrm{p}}_{2}^{*}\right)-\mathrm{F}_{1}\left(\overrightarrow{\mathrm{p}}_{1}\right) \mathrm{F}_{1}\left(\overrightarrow{\mathrm{p}_{2}}\right)\right) \mathrm{d} \overrightarrow{\mathrm{p}}_{2} \text { ada } \mathrm{d} \phi,
\end{aligned}
$$

(149)

which is a form of the Boltzmann collision integral with the Debye-shielded interaction.

Using (135), (137), (145), and (149) in (133), we obtain

$$
\begin{aligned}
& \frac{\partial F_{1}\left(x_{1}\right)}{\partial t}=\left[H_{1}^{o} ; F_{1}\left(x_{1}\right)\right] \\
& +\frac{n}{m} \int_{0}^{2 \pi} \int_{0}^{\infty} \int_{\left(\vec{p}_{2}\right)}\left|\overrightarrow{p_{2}-\vec{p}_{1}}\right|\left(F_{1}\left(\vec{p}_{1}^{*}\right) F_{1}\left(\vec{p}_{2}^{*}\right)-F_{1}\left(\vec{p}_{1}\right) F_{1}\left(\vec{p}_{2}\right)\right) d \vec{p}_{2} \text { ada } d \\
& +\frac{16 \pi^{2} n e^{4}}{(2 \pi)^{3}} \int d \vec{k} d \overrightarrow{p_{2}} \vec{k} \cdot \frac{\partial}{\partial \vec{p}_{1}}\left\{\frac{1}{k^{4}\left|1+L_{+}\left(i \bar{k} \cdot \bar{v}_{1}\right)\right|^{2}}\right.
\end{aligned}
$$




$$
\left.-\frac{1}{\left(\mathrm{k}^{2}+\mathrm{k}_{\mathrm{D}}\right)^{2}}\right\} \delta\left(\overrightarrow{\mathrm{k}} \cdot\left(\overline{\mathrm{v}_{2}-\mathrm{v}_{1}}\right)\right) \overrightarrow{\mathrm{k}} \cdot \overrightarrow{\mathrm{d}}_{12} \mathrm{~F}_{1}\left(\overrightarrow{\mathrm{p}_{1}}\right) \mathrm{F}_{1}\left(\overrightarrow{\mathrm{p}_{2}}\right) .
$$

The collision integral is the same as that used by Allis ${ }^{6}$ and by Spitzer and Härm ${ }^{2}$ insofar as they replace the Coulomb field with a Debye field in adapting the Boltzmann theory to plasmas. It is convenient that the entire effect of close collisions is contained in this term. This is consistent with the concept of the close collision and its analogy with the Boltzmann gas discussed in section 1. 1 .

The integral over $\vec{k}$ in (150) is the contribution to the interaction term which is due to the deviation of the shielding cloud from the Debye shield. This is caused by the nature of $F_{1}$ and the velocity $\bar{v}_{1}$, as can be seen from the fact that

$$
\begin{aligned}
& \lim _{v_{1} \rightarrow 0} L_{+}\left(i \vec{k} \cdot \vec{v}_{1}\right)=\lim _{v_{1} \rightarrow 0} \frac{m \omega_{p}^{2}}{k^{2}} \int_{+} \frac{\vec{k} \cdot \frac{\partial F_{1}\left(\vec{p}_{2}\right)}{\partial \vec{p}_{2}}}{\vec{k} \cdot\left(\frac{v_{1}-v_{2}}{v^{2}}\right)} d \vec{p}_{2} \\
& \rightarrow-\frac{k_{D}^{2}}{k^{2}}(k T) \int_{+} \frac{\frac{\partial F_{1}(u)}{\partial u} d u}{u}
\end{aligned}
$$

For a distribution that is spherically symmetric the integral is real. For a Maxwellian distribution (151) reduces to $\mathrm{k}_{\mathrm{D}}^{2} / \mathrm{k}^{2}$, which makes the $\overrightarrow{\mathrm{k}}$ integral of (150) zero.

If the distribution $F_{1}(p)$ is not Maxwellian, the contribution of the integral over $\vec{k}$ in (150) is of the Fokker-Planck form. If we define $D^{\circ}$ and $B^{O}$ in (5) we have

$$
\begin{aligned}
& \overrightarrow{\mathrm{D}^{\mathrm{o}}} \equiv-\frac{16 \pi^{2} n \mathrm{e}^{4}}{\mathrm{~m}(2 \pi)^{3}} \iint \frac{\mathrm{d} \overrightarrow{\mathrm{p}}_{2} \mathrm{~d} \overrightarrow{\mathrm{k}} \mathrm{k}}{\mathrm{k}^{4}}\left(\frac{1}{\left|1+\mathrm{L}_{+}\left(\mathrm{i} \overrightarrow{\mathrm{k}} \cdot \overrightarrow{\mathrm{v}}_{1}\right)\right|^{2}}-\frac{1}{\left(1+\frac{\mathrm{k}_{\mathrm{D}}^{2}}{\mathrm{k}^{2}}\right)^{2}}\right) \delta\left(\overrightarrow{\mathrm{k}} \cdot\left(\overline{\mathrm{v}_{2}-\mathrm{v}_{1}}\right)\right) \overrightarrow{\mathrm{k}} \cdot \frac{\partial \mathrm{F}_{1}\left(\mathrm{p}_{2}\right)}{\partial \overrightarrow{\mathrm{p}}_{2}} \\
& \overrightarrow{\mathrm{B}^{\mathrm{o}}} \equiv \frac{16 \pi^{2} n \mathrm{e}^{4}}{\mathrm{~m}^{2}(2 \pi)^{3}} \iint \frac{\mathrm{d} \overrightarrow{\mathrm{p}}_{2} \mathrm{~d} \overrightarrow{\mathrm{k}} \overrightarrow{\mathrm{k}} \overrightarrow{\mathrm{k}}}{\mathrm{k}^{4}}\left(\frac{1}{\left|1+\mathrm{L}_{+}\left(\mathrm{i} \overrightarrow{\mathrm{k}} \cdot \overrightarrow{\mathrm{v}}_{1}\right)\right|^{2}}-\frac{1}{\left(1+\frac{\mathrm{k}_{\mathrm{D}}^{2}}{\mathrm{k}^{2}}\right)^{2}}\right) \delta\left(\overrightarrow{\mathrm{k}} \cdot\left(\overline{\mathrm{v}}_{2^{-\mathrm{v}_{1}}}\right)\right) \mathrm{F}_{1}\left(\overrightarrow{\mathrm{p}}_{2}\right) .
\end{aligned}
$$

Armed with solution (150), we are in a position to justify the assumptions (90), (95), (132), and (142). We have shown explicitly that these assumptions are good for $\left|\overrightarrow{Q_{1}-Q_{2}}\right| \ll \lambda_{D}$.

We can now show that the error introduced by these assumptions for $\left|\overrightarrow{Q_{1}-Q_{2}}\right| \sim \lambda_{D}$ is negligible. We are interested in an integral of $\mathrm{F}_{2}\left(\mathrm{x}_{1}, \mathrm{x}_{2}\right)$ over $\overrightarrow{\mathrm{p}}_{2}$ and $\overrightarrow{\mathrm{q}}_{2}$. In this integration consider a value of $\left(\overline{q_{2}-\bar{q}_{1}}\right)$ which is such that $\left|{\overrightarrow{Q_{1}}-\vec{Q}_{2}}\right| \sim \lambda_{D}$ (which also means that $\left.\left|\overrightarrow{\mathrm{q}_{1}-\mathrm{q}_{2}}\right| \sim \lambda_{\mathrm{D}}\right)$. The number of particles at this separation which have undergone a close collision in the past is given by the number of particles that would undergo 
a close collision in an inverse collision starting from the same spacial coordinates with the velocities reversed. The probability for this is given by the solid angle that is available for close collisions and that is given by $A / R^{2}$, where $A$ is the cross section for close collision and $R$ is the initial separation of the particles. For particles separated by $\lambda_{D}$, this ratio is $\left(e^{2} \lambda_{D} / k T\right) / \lambda_{D}^{2}=e^{2} / k T \lambda_{D}$. Since nothing radical happens for these particles, for example, we do not predict a divergent result for their contribution, we may say that the approximations (90), (95), (132), and (142) are valid for all those situations that yield a significant contribution to the kinetic equation for $F_{1}$.

As a final step we shall evaluate the $|\overrightarrow{\mathrm{k}}|$-integration for $\overrightarrow{\mathrm{B}^{\mathrm{O}}}$ and $\overrightarrow{\mathrm{D}^{\mathrm{O}}}$ in (152), which will leave these functions expressed in angular integrals. The $|\vec{k}|$-integration can be performed in the general case as follows. Define

$$
\mathrm{L}_{ \pm}^{\prime}\left(\mathrm{i} \overrightarrow{\mathrm{k}} \cdot \overrightarrow{\mathrm{v}}_{1}\right) \equiv \mathrm{k}^{2} \mathrm{~L}_{ \pm}\left(\mathrm{i} \overrightarrow{\mathrm{k}} \cdot \overrightarrow{\mathrm{v}}_{1}\right)
$$

and note that $L_{ \pm}^{\prime}$ does not depend on $|\vec{k}|$, only on the direction of $\vec{k} / k$. We define

$$
\mathrm{W}\left(\theta, \phi, \overrightarrow{\mathrm{v}}_{1}\right) \equiv \int_{0}^{\infty} \mathrm{dkk^{3 }}\left(\frac{1}{\left|\mathrm{k}^{2}+\mathrm{L}_{+}^{\prime}\left(\mathrm{i} \overrightarrow{\mathrm{k}} \cdot \overrightarrow{\mathrm{v}}_{1}\right)\right|^{2}}-\frac{1}{\left(\mathrm{k}^{2}+\mathrm{k}_{\mathrm{D}}^{2}\right)^{2}}\right)
$$

where $\theta$ and $\phi$ are the polar and azimuthal angles of $\vec{k}$ in $\vec{k}$-space. W converges at the upper limit as a result of the subtraction of the two terms, and the integral in (154) can be evaluated by standard means to yield

$$
\mathrm{W}\left(\theta, \phi, \overrightarrow{\mathrm{v}}_{1}\right)=\operatorname{Re}\left[\frac{L_{+}^{\prime}-L_{+}^{\prime} \log ^{\prime} \frac{L_{+}^{\prime}}{\mathrm{k}_{\mathrm{D}}^{2}}}{L_{+}^{\prime}-L_{-}^{\prime}}\right] .
$$

In terms of $\mathrm{W}$, the functions $\overrightarrow{\mathrm{B}^{\mathrm{O}}}$ and $\overrightarrow{\mathrm{D}^{\mathrm{O}}}$ can be written

$$
\begin{aligned}
& \overrightarrow{D^{\circ}}=-\frac{16 \pi^{2} n e^{4}}{m(2 \pi)^{3}} \int d \vec{p}_{2} \int_{0}^{2 \pi} d \phi \int_{0}^{\pi} \sin \theta d \theta \frac{\vec{k}}{k} W\left(\theta, \phi, \vec{v}_{1}\right) \delta\left(\vec{k} \cdot\left(\overrightarrow{v_{2}-\vec{v}_{1}}\right)\right) \vec{k} \cdot \frac{\partial F_{1}\left(\overrightarrow{p_{2}}\right)}{\partial \overrightarrow{p_{2}}} \\
& \overrightarrow{B^{\circ}}=\frac{16 \pi^{2} n e^{4}}{m^{2}(2 \pi)^{3}} \int d \overrightarrow{p_{2}} \int_{0}^{2 \pi} d \phi \int_{0}^{\pi} \sin \theta d \theta\left(\frac{\vec{k} \vec{k}}{k}\right) W\left(\theta, \phi, \vec{v}_{1}\right) \delta\left(\vec{k} \cdot\left(\overrightarrow{v_{2}-v_{1}}\right)\right) F_{1}\left(\overrightarrow{p_{2}}\right) .
\end{aligned}
$$

For a spherically symmetric distribution $F_{1}(p)$, it is convenient to pick the $z$-axis of the $\overrightarrow{\mathrm{k}}$-integration along the direction of $\overrightarrow{\mathrm{v}}_{1}$. For this case the azimuthal integration yields $2 \pi$, and $\overleftrightarrow{\mathrm{B}^{\circ}}$ and $\overrightarrow{\mathrm{D}^{\circ}}$ are expressed as one-dimensional integrals. The final evaluation of these integrals must, of course, be made with a particular $F_{1}(\bar{p})$.

In the use of (150)-(156) the following should be noted. Although $\vec{B}^{\circ}$ and $\overrightarrow{D^{\circ}}$ are in the form of Fokker-Planck coefficients, they do not represent the complete contribution to the dynamical friction and the diffusion in velocity space. One method of treating the Boltzmann collision integral is to expand the integral in a series of powers of 
deflections in velocity. ${ }^{2,6}$ The coefficients of the first two powers of this expansion will give additional contribution to the dynamical friction and the diffusion in velocity space. The contributions (136) are due to the particular shape of the shielding cloud and happen to be in the form of Fokker-Planck coefficients. 


\section{SUMMARY}

Equation $(150)$ is the result that was sought. As a kinetic equation for $F_{1}(\vec{p})$, it may be considered accurate to first order in $\left(\mathrm{e}^{2} / \mathrm{kT} \lambda_{\mathrm{D}}\right)$. The use of the adiabatic hypothesis has yielded the most general kinetic equation for $\mathrm{F}_{1}$; and insofar as the hypothesis holds, this equation can be said to exist.

The form (150) is appealing because, although it is a new result, it contains terms corresponding to those of various earlier treatments, the Boltzmann equation ( 3 ) and the Fokker-Planck equation (5). (There is no Vlasov type of contribution for the uniform plasma.) Equation 3 was correct for close collisions and in error for grazing collisions; and (5), the opposite. Equation 150 combines these two solutions and forms a bridge between them.

Discussions of the solution of (150) will not be carried out here, since the various terms in (150) correspond to earlier treatments. Spitzer and $\mathrm{Harm}^{2}$ and Allis ${ }^{6}$ have discussed the Boltzmann collision integral with an assumed Debye-shielded interaction. Several authors ${ }^{8-10}$ have obtained the term containing the integral over $f_{2}^{l}$ in (150). This result was (17) mentioned in section 1.2. The divergence in the $\vec{k}$-integration is cancelled by the last term of (150), although the other properties remain the same.

The solution of (150) is still complicated and must be subjected to other approximations. However, within the limitations mentioned above, (150) may be taken to be the equation of evolution of $\mathrm{F}_{1}$, and these approximations of solution may be used and analyzed in their own right. The ability to separate the approximations used in deriving the equation from the approximations used in its solution aids greatly in understanding the accuracy, meaning, and limitations of the analysis. 


\section{APPENDIX}

We shall carry out a derivation of the large-separation solution $\mathrm{f}_{2}^{1}$ which is due to Dupree. ${ }^{11}$ We shall then show that Dupree's result can be put into the form obtained by Lenard. ${ }^{10}$

Dupree considers (97) for $f_{1}^{2}$ with the substitutions $F_{1} \rightarrow S_{-\tau}^{1} F_{1}$ and $D_{0} \rightarrow \frac{\partial}{\partial t}$ as in (66). We write $\vec{q}=\overrightarrow{q_{1}-q_{2}}$ and obtain

$$
\begin{aligned}
& \frac{\partial}{\partial t} f_{2}^{1}\left(\vec{p}_{1}, \vec{p}_{2}, \vec{q} ; S_{-\tau}^{1} F_{1}\right)+\frac{\left(\overline{p_{1}-p_{2}}\right)}{m} \cdot \frac{\partial}{\partial \vec{q}} f_{2}^{1}\left(p_{1}, p_{2}, \vec{q} ; S_{-\tau}^{1} F_{1}\right) \\
& \quad-n \frac{\partial F_{1}\left(\overrightarrow{p_{1}}\right)}{\partial \overrightarrow{p_{1}}} \cdot \frac{\partial}{\partial \vec{q}} \int d \vec{p}_{3} d^{\prime} \vec{q}^{\prime} \psi\left(\overrightarrow{q-q^{\prime}}\right) f_{2}^{1}\left(\vec{p}_{3}, \vec{p}_{2}, \vec{q}^{\prime} ; S_{-\tau}^{1} F_{1}\right) \\
& +n \frac{\partial F_{1}\left(\overrightarrow{p_{2}}\right)}{\partial \vec{p}_{2}} \cdot \frac{\partial}{\partial \vec{q}} \int d \vec{p}_{3} d \vec{q}^{\prime} \psi\left(\overrightarrow{q-q^{\prime}}\right) f_{2}^{1}\left(\vec{p}_{1}, \vec{p}_{3}, q^{\prime} ; S_{-\tau}^{1} F_{1}\right) \\
& =\frac{\partial}{\partial \bar{q}} \psi(\vec{q}) \cdot \vec{d}_{12} F_{1}\left(\overrightarrow{p_{1}}\right) F_{1}\left(\overrightarrow{p_{2}}\right) .
\end{aligned}
$$

This can be written

$$
\left(\frac{\partial}{\partial t}+L_{1}+L_{2}\right) f(\tau)=\frac{\partial}{\partial \vec{q}} \psi(\vec{q}) \cdot \vec{d}_{12} F_{1}\left(\overrightarrow{p_{1}}\right) F_{1}\left(\overrightarrow{p_{2}}\right)
$$

where $L_{1}$ and $L_{2}$ are Landau operators defined in (71) and operate on coordinate 1 and coordinate 2 , respectively. Note that $\mathrm{L}_{1}$ and $\mathrm{L}_{2}$ commute.

The formal solution to $(158)$ is

$$
\mathrm{f}_{2}^{1}\left(\overrightarrow{\mathrm{p}_{1}}, \overrightarrow{\mathrm{p}_{2}}, \overrightarrow{\mathrm{q}}\right)=\int_{0}^{\infty} \mathrm{d} \tau^{\prime} \mathrm{e}^{-\left(\mathrm{L}_{1}+\mathrm{L}_{2}\right) \tau^{\prime}} \frac{\partial}{\partial \overrightarrow{\mathrm{q}}} \psi(\overrightarrow{\mathrm{q}}) \cdot \overrightarrow{\mathrm{d}}_{12} \mathrm{~F}_{1}\left(\overrightarrow{\mathrm{p}_{1}}\right) \mathrm{F}_{1}\left(\overrightarrow{\mathrm{p}_{2}}\right)
$$

where we have taken the limit $\tau \rightarrow \infty$ in agreement with the discussion preceding (98).

It is easier to evaluate

$$
\int \mathrm{d} \overrightarrow{p_{2}} f_{2}^{1}\left(\overrightarrow{p_{1}}, \overrightarrow{p_{2}}, \bar{q}\right)
$$

and to then compute $f_{2}^{l}$ from (157). Using (78) and (79) for the result of the operator $L_{i}$, we have 


$$
\begin{aligned}
& \int \overline{\mathrm{f}}_{1}^{2}\left(\overrightarrow{\mathrm{p}}_{1}, \overrightarrow{\mathrm{p}}_{2}, \overrightarrow{\mathrm{k}}\right) \mathrm{d} \overrightarrow{\mathrm{p}}_{2}=-\frac{1}{(2 \pi)^{2}} \int_{0}^{\infty} \mathrm{d} \tau \int_{-\infty i+\beta}^{\infty j+\beta} \mathrm{d} \sigma_{1} \mathrm{~d} \sigma_{2} \frac{\mathrm{e}^{\left(\sigma_{1}+\sigma_{2}\right) \tau}}{1+\mathrm{L}_{-}\left(-\sigma_{2}\right)} \cdot \int_{-\sigma_{2}+\mathrm{i} \overrightarrow{\mathrm{k}} \cdot \overrightarrow{\mathrm{v}}_{1}} \frac{\mathrm{d} \overrightarrow{\mathrm{p}}_{2}}{} \\
& \left\{\frac{\frac{\mathrm{m} \omega_{\mathrm{p}}^{2}}{\mathrm{k}^{2}}}{1+\mathrm{L}_{+}\left(\sigma_{1}\right)} \frac{\overrightarrow{\mathrm{k}} \cdot \frac{\partial \mathrm{F}_{1}\left(\overrightarrow{\mathrm{p}}_{1}\right)}{\partial \overrightarrow{\mathrm{p}}_{1}}}{\sigma_{1}-\mathrm{i} \overrightarrow{\mathrm{k}} \cdot \overrightarrow{\mathrm{v}}_{1}} \int_{+} \frac{\mathrm{d \textrm {p } _ { 1 }}}{\sigma_{1}-\mathrm{ik} \cdot \overrightarrow{\mathrm{v}}_{1}}+\frac{i}{\sigma_{1}-\mathrm{i} \overrightarrow{\mathrm{k}} \cdot \overrightarrow{\mathrm{v}}_{1}}\right\} \\
& \cdot \frac{4 \pi c^{2}}{k^{2}} \vec{k} \cdot \vec{d}_{12} F_{1}\left(\vec{p}_{1}\right) F_{1}\left(\vec{p}_{2}\right) \text {, }
\end{aligned}
$$

where

$$
\overline{\mathrm{f}}_{2}^{1}\left(\overrightarrow{\mathrm{p}}_{1}, \overrightarrow{\mathrm{p}}_{2}, \overrightarrow{\mathrm{k}}\right)=\int \mathrm{d} \overline{\mathrm{q}} \mathrm{e}^{\mathrm{i} \overrightarrow{\mathrm{k}} \cdot \widehat{\mathrm{q}}_{\mathrm{f}}} \mathrm{f}_{2}^{1}\left(\overline{\mathrm{p}}_{1}, \overrightarrow{\mathrm{p}}_{2}, \overrightarrow{\mathrm{q}}\right)
$$

Equation 159 is the form obtained by Dupree. The $\int_{+} d p$ and $L_{+}$are defined in (78) and (79). The $\int_{-} d p$ and $L_{-}$are the same integrals with the path of integration going under the poles.

As mentioned in connection with (80), the $\sigma$-integration can be moved just to the left of the imaginary axis and the $\tau$-integration carried out. This brings down a $\left(\sigma_{1}+\sigma_{2}\right)^{-1}$ if we can assume $\operatorname{Re} \sigma_{2}<\operatorname{Re} \sigma_{1}$ (if not, carry through the following oper ation with $\sigma_{1}$ and $\sigma_{2}$ interchanged). For the same reasons and conditions that the zeros of $1+L_{+}(\sigma)$ are in the left-half plane, the zeros of $1+L_{-}(-\sigma)$ are also in the left-half plane. Therefore, we close the $\sigma_{2}$ in the right-half plane and have only the contribution at $\sigma_{2}=-\sigma_{1}$. This yields

$$
\begin{aligned}
& \int f_{2}^{1}\left(\vec{p}_{1}, \vec{p}_{2}, \vec{k}\right) d \vec{p}_{2}=\frac{2 c^{2}}{k^{2}} \int_{-\infty i+\beta}^{\infty i+\beta} d \sigma \frac{1}{1+L_{-}(\sigma)} \cdot \int_{-} \frac{d \overrightarrow{p_{2}}}{\sigma-i \vec{k} \cdot \vec{v}_{2}} \\
& \left\{\frac{\frac{m \omega_{p}^{2}}{\mathrm{k}^{2}} \mathrm{ik} \cdot \frac{\partial \mathrm{F}_{1}\left(\overrightarrow{\mathrm{p}}_{1}\right)}{\partial \overrightarrow{\mathrm{p}}_{1}}}{\left[1+\mathrm{L}_{+}(\sigma)\right]\left[\sigma-\mathrm{i} \overrightarrow{\mathrm{k}} \cdot \overrightarrow{\mathrm{v}}_{1}\right]} \int_{+} \frac{\mathrm{d \vec {p } _ { 1 }}}{\sigma-\mathrm{i} \overrightarrow{\mathrm{k}} \cdot \overrightarrow{\mathrm{v}}_{1}}-\frac{1}{\sigma-\mathrm{ik} \cdot \overrightarrow{\mathrm{v}}_{1}}\right\} \\
& \vec{k} \cdot \overrightarrow{d_{12}} F_{1}\left(\overrightarrow{p_{1}}\right) F_{1}\left(\overrightarrow{p_{2}}\right) \text {. }
\end{aligned}
$$

By algebraic manipulations $(160)$ can be put into the form 


$$
\begin{aligned}
\int f_{2}^{1}\left(\vec{p}_{1}, \vec{p}_{2}, \vec{k}\right)= & -\frac{2 c^{2}}{\pi k^{2}} \int_{-\infty i+\beta}^{\infty i+\beta} \frac{d \sigma}{\sigma-i \vec{k} \cdot \vec{v}_{1}} \frac{1}{\left(1+L_{+}(\sigma)\right)\left(1+L_{-}(\sigma)\right)} \int_{-} \frac{d \vec{p}_{2}}{\sigma-i \vec{k} \cdot \vec{v}_{2}} \\
& \cdot\left\{\begin{array}{l}
\left.1+i \frac{m \omega_{p}^{2}}{k^{2}} \vec{k} \cdot \frac{\partial F_{1}\left(\overrightarrow{p_{2}}\right)}{\partial \vec{p}_{2}} \int_{+} \frac{d \vec{p}_{2}}{\sigma-i \vec{k} \cdot \vec{v}_{2}}\right\} \vec{k} \cdot \vec{d}_{12} F_{1}\left(\vec{p}_{1}\right) F_{1}\left(\vec{p}_{2}\right) .
\end{array}\right.
\end{aligned}
$$

In the limit of very small $\beta$

$$
\begin{gathered}
\frac{1}{\mathrm{~m}} \int_{ \pm} \frac{\mathrm{d} \overrightarrow{\mathrm{p}_{2}}}{\sigma-\mathrm{i} \overrightarrow{\mathrm{k}} \cdot \overrightarrow{\mathrm{v}_{2}}}=\frac{1}{\mathrm{~m}} \int_{\sigma} \frac{\mathrm{d} \overrightarrow{\mathrm{p}}_{2}}{-\mathrm{i} \overrightarrow{\mathrm{k}} \cdot \overrightarrow{\mathrm{v}}_{2}} \pm \pi \int \delta\left(\overrightarrow{\mathrm{k}} \cdot \overrightarrow{\mathrm{v}_{2}}+\mathrm{i} \sigma\right) \frac{\mathrm{d} \overrightarrow{\mathrm{p}_{2}}}{\mathrm{~m}} \\
\int_{-\infty i+\beta}^{\infty i+\beta} \frac{\mathrm{d} \sigma}{\sigma-\mathrm{ik} \cdot \mathrm{v}_{1}}=\oint \frac{\mathrm{d} \sigma}{\sigma-i \mathrm{k} \cdot \mathrm{v}_{1}}+\pi \mathrm{i} \int \mathrm{d} \sigma \delta\left(\sigma-\mathrm{i} \overrightarrow{\mathrm{k}} \cdot \overrightarrow{\mathrm{v}_{1}}\right) .
\end{gathered}
$$

Using (162) in (161) with further algebra, we obtain

$$
\begin{aligned}
\int \bar{f}_{2}^{1}\left(\overrightarrow{p_{1}}, \overrightarrow{p_{2}}, \vec{k}\right) d \vec{p}_{2}= & -\frac{2 c^{2}}{k^{2}} \int_{-\infty i+\beta}^{\infty i+\beta} \frac{d \sigma}{\sigma-i \vec{k} \cdot \overrightarrow{v_{1}}} \frac{1}{\left(1+L_{+}(\sigma)\right)\left(1+L_{-}(\sigma)\right)} \\
& {\left[\left(1+L_{-}(\sigma)\right) \int_{+} \frac{d \overrightarrow{p_{2}}}{\sigma-i \vec{k} \cdot \overrightarrow{v_{2}}} \cdot \vec{k} \cdot \overrightarrow{d_{12}} F_{1}\left(\overrightarrow{p_{1}}\right) F_{1}\left(\overrightarrow{p_{2}}\right)\right.} \\
& \left.-2 \pi \int d \vec{p}_{2} \delta\left(\vec{k} \cdot \overrightarrow{v_{2}}+i \sigma\right) \vec{k} \cdot \overrightarrow{d_{12}} F_{1}\left(\overrightarrow{p_{1}}\right) F_{1}\left(\overrightarrow{p_{2}}\right)\right] .
\end{aligned}
$$

The first integral may be closed in the right-half plane with zero contribution because there are no poles in this plane. (By reflection the zeros of $1+L_{-}(\sigma)$ are in the right-half plane because the zeros of $1+L_{-}(-\sigma)$ are in the left-half plane.)

We obtain, as a final result,

$$
\begin{aligned}
\int \overline{\mathrm{f}}_{2}^{-1}\left(\overrightarrow{\mathrm{p}}_{1}, \overrightarrow{\mathrm{p}}_{2}, \overrightarrow{\mathrm{k}}\right) \mathrm{d} \overrightarrow{\mathrm{p}}_{2} \\
=\frac{4 \pi \mathrm{c}^{2}}{\mathrm{k}^{2}} \int_{-\infty \mathrm{i}+\beta}^{\infty \mathrm{i}+\beta} \frac{\mathrm{d} \sigma}{\sigma-\mathrm{i} \overrightarrow{\mathrm{k}} \cdot \overrightarrow{\mathrm{v}}_{1}} \frac{1}{\left(1+\mathrm{L}_{+}(\sigma)\right)\left(1+\mathrm{L}_{-}(\sigma)\right)} \\
\quad \int d \overrightarrow{\mathrm{p}}_{2} \delta\left(\overrightarrow{\mathrm{k}} \cdot \overrightarrow{\mathrm{v}}_{2}+\mathrm{i} \sigma\right) \overrightarrow{\mathrm{k}} \cdot \mathrm{d}_{12} \mathrm{~F}_{1}\left(\overrightarrow{\mathrm{p}}_{1}\right) \mathrm{F}_{1}\left(\overrightarrow{\mathrm{p}}_{2}\right)
\end{aligned}
$$




$$
\begin{aligned}
& =\frac{4 \pi c^{2}}{k^{2}} \int \frac{d \sigma}{\sigma-i \vec{k} \cdot \vec{v}_{1}} \frac{1}{\left|1+L_{+}(\sigma)\right|^{2}} \int d \overrightarrow{p_{2}} \delta\left(\vec{k} \cdot \overrightarrow{v_{2}}+i \sigma\right) \vec{k} \cdot \vec{d}_{12} F_{1}\left(\overrightarrow{p_{1}}\right) F_{1}\left(\overrightarrow{p_{2}}\right) \\
& +i \frac{4 \pi c^{2}}{k^{2}} \frac{1}{\left|1+L_{+}\left(i k \cdot v_{1}\right)\right|^{2}} \int d \overrightarrow{p_{2}} \delta\left(\vec{k} \cdot\left(\overrightarrow{v_{2}-v_{1}}\right)\right) \vec{k} \cdot \vec{d}_{12} F_{1}\left(\overrightarrow{p_{1}}\right) F_{1}\left(\overrightarrow{p_{2}}\right),
\end{aligned}
$$

where we use (162) and the fact that $\left(1+L_{+}(\sigma)\right)\left(1+L_{-}(\sigma)\right)=\left|1+L_{+}(\sigma)\right|^{2}$ for $\sigma$ that is pure. imaginary.

Fortunately the principal value term in (163) is even in $\vec{k}$ while the second term is odd. Thus in the integral

$$
n \int\left[\psi_{12} ; f_{2}^{1}\left(\vec{p}_{1}, \vec{p}_{2}, \overline{q_{1}-q_{2}}\right)\right] d \vec{p}_{2} d \vec{q}_{2}=-\frac{m \omega_{p}^{2}}{(2 \pi)^{3}} \int \frac{d \vec{k}}{k^{2}} \vec{k} \cdot \frac{\partial}{\partial \overrightarrow{p_{1}}} \int \bar{f}_{2}^{l}\left(\overrightarrow{p_{1}}, \overrightarrow{p_{2}}, \vec{k}\right) d \overrightarrow{p_{2}}
$$

we need only the second term.

Introducing (163) into (164), we obtain (17) for the evolution of a spacially homogeneous $\mathrm{F}_{1}$,

$$
\frac{\partial \mathrm{F}_{1}\left(\overrightarrow{\mathrm{p}}_{1}\right)}{\partial \mathrm{t}}=\frac{16 \pi^{3} \mathrm{nc} \mathrm{c}^{4}}{(2 \pi)^{3}} \iint \frac{\mathrm{d} \overrightarrow{\mathrm{k}} \mathrm{d} \overrightarrow{\mathrm{p}}_{2}}{\mathrm{k}^{4}} \overrightarrow{\mathrm{k}} \cdot \frac{\partial}{\partial \overrightarrow{\mathrm{p}}_{1}} \frac{\delta\left(\overrightarrow{\mathrm{k}} \cdot\left(\overrightarrow{\mathrm{v}_{2}-\mathrm{v}_{1}}\right)\right)}{\left|1+\mathrm{L}_{+}\left(\mathrm{i} \overrightarrow{\mathrm{k}} \cdot \overrightarrow{\mathrm{v}}_{1}\right)\right|^{2}} \overrightarrow{\mathrm{k}} \cdot \overrightarrow{\mathrm{d}}_{12} \mathrm{~F}_{1}\left(\overrightarrow{\mathrm{p}_{1}}\right) \mathrm{F}_{1}\left(\overrightarrow{\mathrm{p}_{2}}\right) .
$$

\section{Acknowledgment}

The author wishes to express his appreciation to Professor William P. Allis, for his patience and useful advice during the preparation of his thesis. He would also like to thank Professor Thomas H. Dupree for interesting discussions of the material. He would like to thank the National Science Foundation for support extended during the course of this investigation. 


\section{References}

1. J. Kirkwood and J. Poirier, J. Phys. Chem. 58, 591 (1954).

2. L. Spitzer, Jr. and R. Härm, Phys. Rev. 89, 977 (1953).

3. A. Vlasov, J. Expt1. Theoret. Phys. (U.S.S.R.) $\underline{8}, 291$ (1938).

4. L. Landau, J. Phys. (U.S.S. R.) 10, 25 (1946).

5. S. Chandrasekhar, Revs. Modern Phys. 15, 1 (1943).

6. W. P. Allis, Motions of Ions and Electrons, Handbuch der Physik, Vol. 21, edited by S. Flügge (Springer Verlag, Berlin, 1956), pp. 384-444; Technical Report 299, Research Laboratory of Electronics, M.I. T., June 13, 1956.

7. R. Balescu, Phys. Fluids $\underline{3}, 52$ (1960).

8. C. Tchen, Phys. Rev. 114, 394 (1959).

9. N. Rostocker and M. Rosenbluth, Phys. Fluids $\underline{3}, 1$ (1960).

10. A. Lenard, Ann. Phys. 3 , 390 (1960).

11. T. Dupree, Phys. Fluids $\underline{4}, 696$ (1961).

12. N. Bogobiubov, Problems of Dynamic Theory in Statistical Physics (Leningrad, 1946); Translation, L. Venters, AEC-tr-3852.

13. G. Baccus, J. Math. Phys. 1, 178 (1960).

14. J. Drummond, Plasma Physics (McGraw-Hill Book Company, Inc., New York, 1961), Chapter 1.

15. Bateman Manuscript Project, California Institute of Technology, Tables of Integral Transforms, Vol. I (McGraw-Hill Book Company, Inc., New York, 1954), Chapter 7.

16. Bateman Manuscript Project, California Institute of Technology, Higher Transcendental Functions, Vol. 2 (McGraw-Hill Book Company, Inc., New York, 1954). 
.

-

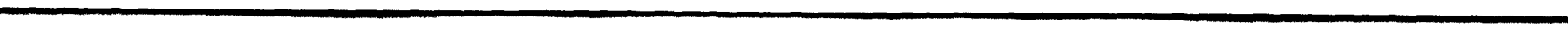

